\title{
Review on Current Sheets in CME Development: Theories and Observations
}

\author{
Jun Lin ${ }^{1}$ - Nicholas A. Murphy ${ }^{2}$ Chengcai Shen ${ }^{2}$. \\ John C. Raymond ${ }^{2} \cdot$ Katharine K. Reeves ${ }^{2}$. \\ Jiayong Zhong ${ }^{3} \cdot \mathrm{Ning} \mathrm{Wu}^{4} \cdot$ Yan $\mathrm{Li}^{1}$
}

Received: 20 September 2014 / Accepted: 10 October 2015 / Published online: 26 October 2015

(C) The Author(s) 2015. This article is published with open access at Springerlink.com

\begin{abstract}
We introduce how the catastrophe model for solar eruptions predicted the formation and development of the long current sheet (CS) and how the observations were used to recognize the CS at the place where the CS is presumably located. Then, we discuss the direct measurement of the CS region thickness by studying the brightness distribution of the CS region at different wavelengths. The thickness ranges from $10^{4} \mathrm{~km}$ to about $10^{5} \mathrm{~km}$ at heights between 0.27 and $1.16 \mathrm{R}_{\odot}$ from the solar surface. But the traditional theory indicates that the CS is as thin as the proton Larmor radius, which is of order tens of meters in the corona. We look into the huge difference in the thickness between observations and theoretical expectations. The possible impacts that affect measurements and results are studied, and physical causes leading to a thick CS region in which reconnection can still occur at a reasonably fast rate are analyzed. Studies in both theories and observations suggest that the difference between the true value and the apparent value of the CS thickness is not significant as long as the CS could be recognised in observations. We review observations that show complex structures and flows inside the CS region and present recent numerical modelling results on some aspects of these structures. Both observations and numerical experiments indicate that the downward reconnection outflows are usually slower than the upward ones in the same eruptive event. Numerical simulations show that the complex structure inside CS and its temporal behavior as a result of turbulence and the Petschek-type slow-mode shock could probably account for the thick CS and fast reconnection. But whether the CS itself is that thick still remains unknown since, for the time being, we cannot measure the electric current directly in that region. We also review the most recent laboratory experiments of reconnection driven by energetic laser beams, and discuss some important topics for future works.
\end{abstract}

\section{J. Lin}

jlin@ynao.ac.cn

1 Yunnan Observatories, Chinese Academy of Sciences, P.O. Box 110, Kunming, Yunnan 650216, China

2 Harvard-Smithsonian Center for Astrophysics, 60 Garden Street, Cambridge, MA 02138, USA

3 Key Laboratory of Optical Astronomy, National Astronomical Observatories of China, Chinese Academy of Sciences, 20A Datun Road, Chaoyang District, Beijing 100012, China

4 School of Tourism and Geography, Yunnan Normal University, 293 Street 121, Kunming, Yunnan 650092, China 
Keywords Sun: flares $\cdot$ Sun: coronal mass ejections (CMEs) $\cdot$ Plasmas $\cdot$ Magnetic reconnection · Instabilities

\section{Introduction}

Magnetic reconnection is a ubiquitous process in magnetized plasmas, in which magnetic energy is converted into heat and kinetic energy of the plasma, and the kinetic energy of energetic particles. In the simplest picture of this process, two plasmas with magnetic fields of opposite polarity are brought together, producing a magnetically neutral region, or a neutral point between the two fields. Magnetic field lines quickly break and reconnect, and release the magnetic energy. When stretched or squeezed, the neutral region collapses to a current sheet (CS; see also Figs. 2.1 and 2.2 of Priest and Forbes 2000, which show the evolution in various magnetic configurations including an X-type neutral point, also known as the $\mathrm{X}$-point, and/or a CS) where magnetic reconnection takes place.

Usually, in the solar and the other astrophysical environments, we may not be able to pin down mechanism for reconnection in a specific event due to the limited observations, and also due to the fact that even the detail in the reconnection region theoretically is still an open question. Different mechanisms could give rise to the same output. For example, Sweet-Parker CS with anomalous resistivity (Priest 1982; Biskamp 2000; Priest and Forbes 2000), Petschek-type reconnection (Petschek 1964; Biskamp 1986; Forbes and Priest 1987; Yokoyama and Shibata 1994; Kulsrud 2001; Drake et al. 2006; Baty et al. 2009; Vršnak et al. 2009; Zweibel and Yamada 2009; Ko et al. 2010; Liu et al. 2012), and turbulent reconnection (Strauss 1988; Bhattacharjee and Yuan 1995; Lazarian and Vishniac 1999; Lin et al. 2007, 2009; Lazarian et al. 2012, 2014). These different dissipation styles may even work together to yield a much more complex pattern and more efficient diffusion than that dominated only by a single mechanism (e.g., see also Mei et al. 2012). Therefore, in the present work, the term of "current sheet" should be understood in a more general sense than was used traditionally such that it refers to any region or structure in which magnetic diffusion occurs or has occurred rapidly.

In the solar eruptive process, the closed magnetic field is severely stretched, and the neutral region generally appears in the form of a CS (e.g., see also Priest and Forbes 2002; Lin et al. 2003; Forbes et al. 2006). Magnetic reconnection through the CS helps the magnetized plasma ejected by the eruption propagate away smoothly and produces a flare near the solar surface. Usually, the eruption happens in an environment where the electrical conductivity of the plasma and the length scale of the magnetic field are fairly large, so rapid dissipation of the magnetic field is almost impossible (e.g., Priest 1982; Priest and Forbes 2000) unless the thickness of the CS or quasi-separatrix layers (e.g., Démoulin et al. 1996; Priest and Forbes 2000) is small so that fast magnetic reconnection could occur easily.

The magnetic configuration in the solar atmosphere in which magnetic reconnection occurs and two-ribbon flares are produced was suggested by Carmichael (1964), and the famous Kopp-Pneuman model for the two-ribbon flare (Fig. 1) was developed on the basis of Carmichael's original idea (e.g., see Kopp and Pneuman 1976; Švestka 1976; Švestka and Cliver 1992). In this model, energy is stored in a force free magnetic arcade prior to the eruption. Eventually, the field erupts to form a fully open magnetic configuration including a CS due to instabilities in the system (Fig. 1a). The open configuration then relaxes into a closed, nearly potential field via magnetic reconnection in the CS, releasing the magnetic energy stored in the system beforehand, and returns to the initial closed structure. This implicitly suggests that the magnetic energy stored is mainly released by the 
(a)

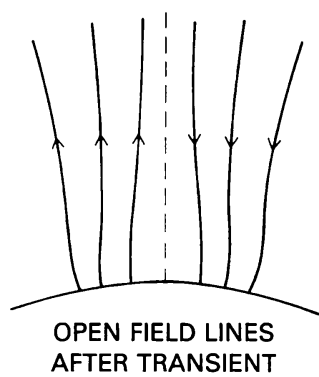

(b)

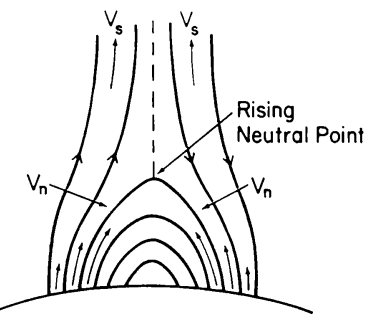

SUBSEQUENT RECONNECTION WITH CAPTURE OF MATERIAL ON CLOSED FIELD LINES

Fig. 1 Postflare/CME loop model by Kopp and Pneuman (1976). (a) The magnetic field is pushed open by an eruption, and a current sheet separates two antiparallel magnetic field lines. (b) The opened configuration relaxes into a closed, nearly potential field via magnetic reconnection in the current sheet. This process produces two bright and separating flare ribbons on the solar disk and a continually growing flare loop system in the corona (reproduction of Fig. 1 of Kopp and Pneuman 1976)

post-eruption reconnection process (e.g., see also Forbes et al. 1994). The clear manifestation of this process in observations is a bright post-flare loop in the corona and a pair of separating bright ribbons on the solar disk, for which the "two-ribbon flare" was named. Several early versions of the two-ribbon flare configuration could be found in the work of Švestka and Cliver (1992). It is now known that the motions of the flare ribbons and loops are not due to mass motions of the plasma but rather due to the upward propagation of the energy source onto new magnetic field lines at higher altitudes (Schmieder et al. 1987); the hot X-ray loops are the newly reconnected closed loops, located at the outermost edge of the loop system, mapping to the outer edge of the flare ribbons; while the cool $\mathrm{H} \alpha$ loops are formed from the hot ones by conductive and radiative cooling (see Ko et al. 2003 and references therein for a brief review on this issue). (Note: The term "altitude" used in this paper means the distance measured from the surface of the Sun.)

Because of the approximate force-free environment in the low corona, the open or semiopen magnetic structure required for the Kopp-Pneuman model cannot exist unless disruption occurs and the closed magnetic field is quickly stretched outward. According to this basic physical properties of this process, Forbes and co-workers (e.g., see Forbes and Isenberg 1991; Isenberg et al. 1993; Forbes et al. 1994; Forbes and Priest 1995) investigated in detail how a closed coronal magnetic configuration could evolve to a Kopp-Pneuman-type configuration. They pointed out that the coronal magnetic field continues to evolve in response to the motion of plasma in the photosphere, and the kinetic energy of mass motions in the photosphere is thus converted into the magnetic energy that is stored in the coronal magnetic field. Usually, this process takes tens of hours, even days, and the coronal magnetic field evolves quasi-statically until the energy stored in the corona exceeds the threshold and the system evolves to the critical point.

Generally, the amount of energy stored in the coronal magnetic configuration is a critical parameter used for describing the equilibrium state of the system (see discussions of Forbes 1991). Equivalently, on the other hand, other parameters for the property of the photospheric boundary conditions may also constitute the critical parameter of the catastrophe model (see detailed discussions by Forbes and Isenberg 1991 and Lin et al. 2003). These parameters include the total magnetic flux sent from the photosphere to the corona (Forbes and Isenberg 1991), strength of the background field (Isenberg et al. 1993; Lin et al. 1998), distance between the source regions on the photosphere (e.g., see Forbes and Priest 1995), and so on. 
As the system evolves to the critical point, further evolution in or a perturbation to the configuration causes the loss of equilibrium in the system, and the consequent evolution becomes dynamic. Then the magnetic energy stored in the system is quickly released, giving rise to the eruption. The energy release often lasts from several tens of minutes to many hours. The transition from the quasi-static to dynamic evolution constitutes the catastrophe, and the corresponding model for solar eruptions are known as the catastrophe model (see Lin et al. 2003 for more details). Alternative models to the catastrophe one for triggering eruptions include the sheared arcade model (Mikić et al. 1988; Mikić and Linker 1994; Linker et al. 2003; Amari et al. 2005, 2010; Reeves et al. 2010), the breakout model (Antiochos et al. 1999; Lynch et al. 2010; Karpen et al. 2012), the ideal MHD model on the basis of the kink and torus instability (Titov and Démoulin 1999; Török and Kliem 2005; Kliem and Török 2006; Fan and Gibson 2007; Kliem et al. 2010; Karlický and Kliem 2010), the tether-cutting model (Moore et al. 2001), and so on (see also Shibata and Magara 2011; Yang et al. 2012; Schmieder et al. 2013; Yan et al. 2014).

In the sheared arcade model, a set of simply-connected force-free magnetic arcades are sheared, and the arcades expand outward gradually, a CS develops between two magnetic fields of opposite polarity, and the evolution in the system turns into dynamic as the diffusion in the CS is invoked. In the breakout model, the magnetic configuration has a quadrupolar geometry. Shearing the central arcade causes this part of magnetic field to expand outward and interact with the background field overhead, and magnetic reconnection eventually results in the loss of the equilibrium in the system. In the ideal MHD model on the basis of the kink and torus instability, a toroidal electric current channel could be in the tokamak equilibrium (or Shafranov equilibrium, Shafranov 1966) as the magnetic tension due to the background magnetic field and the magnetic compression due to the curvature of the current channel balance one another (see also Lin et al. 1998, 2002), and the equilibrium is lost as the balance breaks down. Recently, Kliem et al. (2014) confirmed the equivalent role of the kink and torus instability to the catastrophic loss of equilibrium in triggering the eruption, and further pointed out that in both frameworks, the equilibrium in system could be driven to non-equilibrium through the tether-cutting process; Longcope and Forbes (2014) have also found that a flux rope in quadrupolar external field could reach a catastrophe along various evolutionary paths, depending on the detailed form of the initial equilibrium.

Basically, all these models (theories) of solar eruptions are fundamentally similar, or even the same, such that the energy driving the eruption is stored in the coronal magnetic field beforehand, and the loss of equilibrium in the system triggers the eruption. The difference lies only on the fact that magnetic reconnection is not necessary in triggering the loss of equilibrium in the catastrophe (equivalently the kink and torus instability) model, and it is needed in the other models. The consequent evolutionary features in the disrupting configuration is the same in either model after the loss of equilibrium has occurred (e.g., see also detailed discussions of Lin et al. 2003). In the present work, we follow the catastrophe model for simplicity.

Because the coronal magnetic field is line-tied to the dense photosphere (see the definition and discussions given by Forbes 1991), the closed magnetic field in the lower corona is severely stretched during the catastrophe, creating a CS between two fields of opposite polarity and thrusting the upper part of the magnetic configuration (usually including a filament or current-carrying flux rope) outward. In the ideal magnetohydrodynamic (MHD) environment, the CS is infinitely thin and attached to the photosphere. The increasing magnetic tension due to the magnetic field lines passing over the flux rope and anchoring to the photosphere tries to halt the outward motion of the flux rope, and the evolution of the system will be eventually stopped after the flux rope (or the whole system) finds the new 
equilibrium state. This implies that the catastrophe cannot develop to a plausible eruption in the ideal MHD environment (see also Lin et al. 2003 for detailed discussions).

In the real coronal environment, on the other hand, the electric resistivity of the Spitzer type is very small, but not zero. So finite diffusion exists although it is indeed very tiny. Therefore, dissipation inevitably occurs, especially in the CS. Since the resistivity is small and the catastrophe takes place much faster than the dissipation at the beginning of the eruption (see discussions of Forbes and Lin 2000; Lin and Forbes 2000; Lin 2002), the CS is able to develop to a large scale, and various plasma instabilities would occur in the CS. Linear theory of the tearing mode instability indicates that the CS becomes unstable as its length exceeds $2 \pi$ times its thickness (Furth et al. 1963; Priest and Forbes 2000), and nonlinear investigations through numerical experiments suggest that this ratio could apparently exceed 10 and even up to 100, before the tearing mode is invoked (e.g., see Loureiro et al. 2007; Ni et al. 2010; Shen et al. 2011; Bárta et al. 2011a, 2011b; Mei et al. 2012). Plasma instabilities yield turbulence inside the CS that may significantly enhance the dissipation of the magnetic field, resulting in an effective resistivity that is much higher than the traditional Spitzer value (Strauss 1986, 1988; Ambrosiano et al. 1988; Bhattacharjee and Yuan 1995; Shibata and Tanuma 2001; Skender and Lapenta 2010; Bárta et al. 2011a, 2011b; Shen et al. 2011; Mei et al. 2012; and references therein). The enhanced resistivity accelerates the dissipation in the CS in the form of fast reconnection, which causes the growth of the flare loops below the CS and the rapid expansion of the CME bubble above the CS (see Fig. 2, and Lin et al. 2004; Lin and Soon 2004). Hence, the disrupting magnetic field does not necessarily become fully open before the CME propagates outward from the relevant coronal magnetic configuration.

The scenario sketched by Fig. 2 suggests that the role played by reconnection in an eruptive process is twofold. First, reconnection breaks the magnetic field lines that pass over the flux rope and are anchored in the boundary surface at both ends. These field lines produce strong magnetic tension in a stretched configuration that would otherwise prevent the flux rope from escaping to form a CME. [We note here that, in most cases, breaking magnetic field lines could either take place at the top of the magnetic arcade by reconnection between the arcade and the overlying background field as the break-out model described (e.g., see Antiochos et al. 1999 and Fig. 3), or take place by reconnection through the CS between the flux rope and the flare as described by the catastrophe model (e.g., see detailed described by Forbes and Lin 2000; Lin and Forbes 2000; Lin 2002; Lin et al. 2003, as well as the two thick blue arrows in Fig. 2). On the other hand, works of Aurass et al. (2013) and Aurass (2014) have convincingly showed that both the reconnection sites could be observed simultaneously in the same event. Combinations of $X$-ray and metric radio spectroscopy and imaging of their data revealed structures and electron acceleration sites at locations beyond the SDO/AIA and SOHO/EIT FOVs but under the edge of the occulting disk of LASCO/C2. However, obtaining such observational data is not trivial, only when the event takes place near, but not at, the limb of the Sun, can it become possible. In the present work, we follow the case of the catastrophe as shown in Fig. 2].

Second, reconnection dumps large amounts of energy in the lower atmosphere of the Sun, yielding intense heating, which accounts for the traditional flare ribbons and loops. Figure 2 incorporates the flare models summarized by Forbes and Acton (1996) and the CME model of Lin and Forbes (2000), and clearly illustrates the relationship among solar flares, eruptive prominences, and CMEs such that these three phenomena are different manifestations of a single physical process that involves a disruption of the coronal magnetic field (see also Martens and Kuin 1989; Priest and Forbes 2002). On the other hand, the scenario described by Fig. 2 is usually valid for the major eruptions that produce both solar flares and CMEs, and it may not be good for events that produce either a flare or CME alone. 
Fig. 2 Upper part: Sketch of the flux rope/CME model of Lin and Forbes (2000) showing the eruption of the flux rope, the current sheet formed behind it, and the postflare/CME loops below, as well as the inflows and outflows associated with the reconnection. Lower part:

Enlarged view of the postflare/CME loops (adopted from Forbes and Acton 1996). The upper tip of the cusp rises as reconnection happens continuously

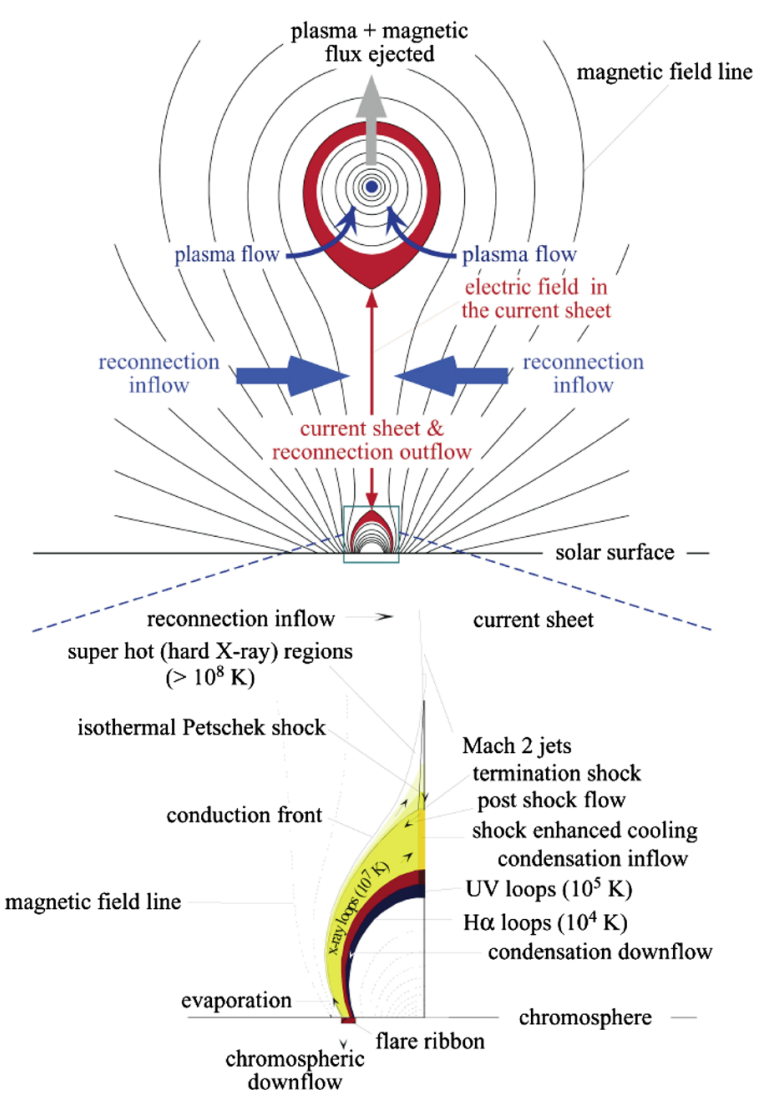

The development of a long CS following the onset of the eruption is a very important prediction of the Lin and Forbes (2000) model. Because the timescale of reconnection is long compared to the timescale of the onset stage (Alfvén timescale), a long CS would be expected in the eruption even though reconnection is considered fast. The highest altitude flare loops reach and the distance that the rear part of the CME propagates during the typical eruptive event have indirectly confirmed the formation of the long CS (see discussions of Ciaravella et al. 2002; Lin 2002; Ko et al. 2003; Lin et al. 2005).

The formation of a CS behind the CME was half-quantitatively predicted, for the first time, by Martens and Kuin (1989), and then quantitatively described by Lin and Forbes (2000) such that a long CS could be expected in the major eruption. The other models for the eruption, like the sheared-arcade model (Mikić et al. 1988; Mikić and Linker 1994) and the breakout model (Antiochos et al. 1999) in which reconnection creates the flux rope, did not explicitly address the development and the other related features/properties of the CS behind the CME probably because either the numerical resistivity was high and the CS is hard to form in the numerical experiments, or the main attention was paid to the triggering of the eruption. In the follow-ups of these works, on the other hand, with the improvement in the numerical techniques and more and more attention being paid on the CS structure, the related works also showed the appearance of a long CS behind the CME in these models, and detailed properties of the CS started to be investigated (e.g., see Linker et al. 2003; Riley et al. 2007; Reeves et al. 2010; Karpen et al. 2012 for details). Therefore, a CS behind the 


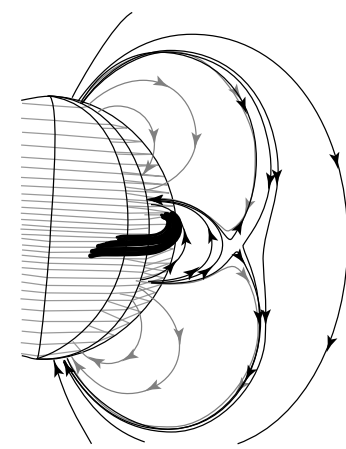

(a)

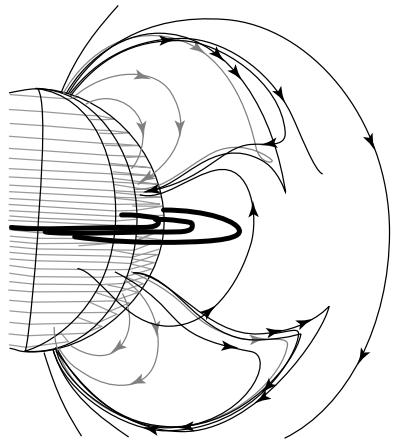

(b)

Fig. 3 Evolution in the magnetic configuration of the breakout model (Antiochos et al. 1999). Because of the symmetry about the rotation axis, one side is shown. Shearing the arcade field (thick lines) creates a force-free current at the equator, and that the sheared region bulges outwards produces a toroidal current layer as well. Reconnection of the field lines in this layer allows the sheared field lines to open outward to infinity. (a) Magnetic configuration at early stage, and (b) magnetic configuration at late stage. From Forbes and Lin (2000)

CME is a common feature present in all three models for solar eruptions, namely shearedarcade, breakout, and catastrophe models.

In addition to these works that considered development of a single CS during the eruptive process assuming that the destabilization of the magnetic configuration is mainly caused by the evolution of the background field, another set of studies takes into account the role of the interaction between a destabilized structure in an active region with overlying closed magnetic fields via coronal magnetic breakout reconnection (e.g., see Antiochos 1998 for the theory, and Antiochos et al. 1999; Lynch et al. 2004, 2008, 2010; Zhang et al. 2005, 2006; Zhang and Wang 2007 for simulations). They found that the interaction between two systems of magnetic configuration of different topology results in the formation of a CS parallel to the solar surface, and the disruption of the destabilized structure eventually yields the formation of another CS perpendicular to the surface (see Fig. 3 as an example). The latter CS is also known as the "flare CS" since magnetic reconnection occurring inside directly accounts for the solar flare. Therefore, two CS could be expected to develop in the eruption in the framework of this type of model, namely the "breakout CS", and the "flare CS" is expected to dominate the energetics. As shown by Fig. 3, the breakout CS always appears on the top of the central magnetic arcade above a certain height in the middle corona, and directly penetrates into the $\mathrm{CME}$ bubble.

Aurass et al. (2013) observationally verified that this process works from the very beginning of the impulsive flare phase. Using meter wave imaging and spectroscopy these authors have shown that the eruption started almost simultaneously with the formation of two CS perpendicular to each other: the flare CS at low heights, and an azimuthally extended breakout CS at about $0.4 \mathrm{R}_{\odot}$ in front of the flare CS. In a more recent work by Aurass (2014), the first observation is presented of the reverse coronal breakout (the closure of the coronal field behind the CME body). These two works also further confirmed that the breakout CS exists in a certain height range and is penetrating into the CME bubble.

For the first time, Yokoyama et al. (2001) identified the reconnection inflow above the top of the flare loop system, and Ciaravella et al. (2002) provided spectroscopic evidence 
of the CME/flare CS by displaying a narrow and hot region between a CME and the associated flare. This region was at the position where the CS is presumably located (see also Fig. 2). Webb and Cliver (1995) found evidence for magnetic disconnection in the form of concave-outward features in white-light images, and Webb et al. (2003) then analyzed the data from the Solar Maximum Mission (SMM) satellite, and noticed that many CMEs were followed by a long ray-like structure in white light, and they identified this structure with the CS. Ko et al. (2003) studied the emission features and properties of the CS in detail, identified several plasma blobs flowing away from the Sun along the CS and measured the speed of the reconnection outflow and the rate of magnetic reconnection as well as the magnetic field strength near the CS. Lin et al. (2005) performed direct measurement of the reconnection inflow speed near the CS, identified five moving plasma blobs to obtain the reconnection outflow speed and the rate of reconnection. McKenzie and Hudson (1999), Innes et al. (2003a, 2003b), Asai et al. (2004), Sheeley et al. (2004), Reeves et al. (2008a), and Savage and McKenzie (2011) studied the sunward reconnection outflow above the flare loops and recognized fine structures of the plasma flow, also known as the supra-arcade downflow (SAD).

The reconnection outflows play an important part in the structure of the CME. Numerical experiments performed by Riley et al. (2007) displayed bi-directional reconnection outflows along the CS in the solar eruptive events. Raymond et al. (2003), Lin et al. (2004), Lin and Soon (2004), and Bemporad et al. (2006) investigated the consequences of the reconnection process manifested by the thermal structures and kinetic behaviors of CMEs. They found that the CME bubble is surrounded by the hot reconnected plasma, and that the rapidly expanding CME bubbles are mainly due to the addition of magnetic flux brought by the reconnection outflow from the CS into the CME. Lin et al. (2004) and Lin and Soon (2004) further pointed out that it is the anti-sunward reconnection outflow that accounts for the three-component structure of the CME such that the mass and the associated magnetic field in the core of the CME come from the prominence before the eruption, and those surrounding the core are first brought into the CS by the reconnection inflow from the corona nearby, and then sent into the CME bubble around the core by the reconnection outflow. Observations of STEREO confirmed this scenario (e.g., see Davis et al. 2009), and indicated that the three-component structure of the CME could even be observed to a very large distance from the Sun (e.g., see Fig. 2 of Davies et al. (2009), which described mass flows from the Sun, including the $\mathrm{CME}$ ).

Meanwhile, more and more attention has now been drawn to the CS, and studies on the topic have been continuously pushed forward. In this review, we introduce observational features of structures associated with the CS and related results of investigations, discussing studies and results in both theories and numerical experiments on the CS, and then pointing out the importance of these results to our understanding and knowledge of the CS connecting a CME to the associated flare, as well as properties of magnetic reconnection occurring in such a region. We display the first set of observational works on the CS, including identifications of the CS shortly after the prediction of Lin and Forbes (2000) and observational features of reconnection inside and around the CS in Sects. 2 and 3. Table 1 contains several highly valuable observations that are discussed in this work; the related events are listed in the order that they appear in the text for the reader's reference. Observational studies regarding the internal structure and the scale of the CS will be introduced in Sect. 4, and numerical experiments on the relevant topics and the results are unfolded in Sect. 5. We finally summarize this review in Sect. 6. 
Table 1 Dates of the events discussed in this work and the section(s) where they are mentioned

\begin{tabular}{llll}
\hline Event date & Section(s) & Event date & Section(s) \\
\hline 23 Mar 1998 & $2,4.1$ & 03 Nov 2010 & 3.1 \\
21 Apr 2002 & $2,3.2$ & 17 Aug 2011 & $2,3.2$ \\
20 Nov 1988 & 2 & 20 Jan 1999 & $2,3.2$ \\
23 Jul 2002 & $2,3.2$ & 08 Jan 2002 & $2,3.3,4.1$ \\
24 Aug 2002 & 2 & 09 Apr 2008 & $2,3.4$ \\
26 Nov 2002 & 2 & 18 Aug 2010 & 3.4 \\
28 Jul 2004 & 2 & 17 May 2002 & 2 \\
01 Dec 2004 & 3.4 & 03 Nov 2003 & 2 \\
26 Jun 2005 & 4.1 & 18 Mar 1999 & 3.1 \\
04 Nep 2005 2003 & $2,4.1$ & 18 Nov 2003 & $2,3.1,3.3,4.1$ \\
28 Oct 2003 & 4.2 & 29 Jul 2004 & $3.1,3.3$ \\
\hline
\end{tabular}

\section{Identifications of the Current Sheet in the Solar Eruptive Events}

Usually, direct observations of the current sheet are very hard, if not impossible. The high electrical conductivity and nearly force free environment confine the current sheet to a very local region in the direction perpendicular to its vertical extent (refer to Fig. 2). Thus, its thickness is usually small compared to the size of nearby large-scale structures. In addition, the low plasma density in the corona implies that the material inside the CS should be tenuous as well. Therefore, it is not easy to observe the current sheet in an eruptive process because both its size and emission are easily dominated by large-scale and bright structures nearby (see our detailed discussions below and those given by Forbes and Acton 1996) although the CS is indeed a common feature developed in the major eruption as we mentioned earlier. Using data from Yohkoh and SOHO, Švestka (1996), Švestka et al. (1998), McKenzie and Hudson (1999), and McKenzie (2000) studied behaviors of regions above the top of the flare loop system, and displayed typical characteristics of the reconnection region and revealed important information above the reconnection process.

Ciaravella et al. (2002) analyzed spectral data obtained from the Ultraviolet Coronagraph Spectrometer (UVCS; Kohl et al. 1995) on SOHO for an eruption on 1998 March 23. This event developed a slow CME propagating at speed of $100 \mathrm{~km} \mathrm{~s}^{-1}$ when the CME core was at the heliocentrical distance (namely the distance measured from the center of the Sun) of $3.5 \mathrm{R}_{\odot}$, and it reached a speed of $180 \mathrm{~km} \mathrm{~s}^{-1}$ as the core was at $15 \mathrm{R}_{\odot}$. It is usual for slow CMEs to accelerate to a distance of several solar radii (Sheeley et al. 1999). Meanwhile, flare loops in the low corona were observed by EIT in $304 \AA$, and emission from the high temperature coronal forbidden lines [Fe XVIII] (maximum emission occurs at temperature of $6 \times 10^{6} \mathrm{~K}$ ) and [Ca XIV] (maximum emission occurs at temperature of $4 \times 10^{6} \mathrm{~K}$ ) were identified by UVCS between the flare loops and the CME core.

Together with these two very high temperature lines, emission from some other active region spectral lines, such as Fe XVII and Ne IX were also observed. But the emission from the ordinary lines (namely the spectral lines that can usually be observed in the quiet corona) was dispersed along the UVCS slit, and the emission from [Fe XVIII] and [Ca XIV] appeared in a narrow and bright region. Figure 4 displays a composite of EIT (304 ̊̊), LASCO/C2, and UVCS slit images. The UVCS slit image indicated by the arrow is the true location of 


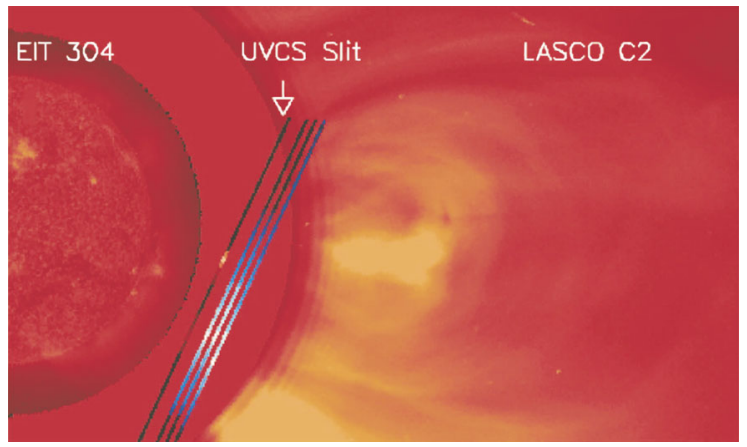

Fig. 4 Composite image of EIT $304 \AA$ (22:24 UT), LASCO C2 (12:33 UT), and line intensity distribution along the UVCS (16:56 UT) slit taken on 1998 March 23. The arrow indicates the true position of the UVCS entrance slit, and the intensity distribution along it is that of the [Fe XVIII] $\lambda 974$ line. The other three slits were plotted for comparison and show (starting from the left) the line intensity distribution of Si XII $\lambda 499$, $\mathrm{O}$ VI $\lambda 1032$, and Ly $\beta$. The UVCS data used in this figure were taken on March 23 with the slit pointed at $1.45 \mathrm{R}_{\odot}$ and $\mathrm{PA}=245^{\circ}$. The position of the peak in the [Fe XVIII] line is PA $=257^{\circ}$ with height $1.55 \mathrm{R}_{\odot}$. The different times were chosen to combine the data into a single image. At the time of the UVCS observation, the LASCO CME was much larger but similar in shape. From Ciaravella et al. (2002)

the slit when [Fe XVIII] and [Ca XIV] emission was detected, and the other three slits are shown for comparison. The narrow and bright region in [Fe XVIII] and [Ca XIV] emission is located below the CME observed by LASCO/C2, and above the flare loop system observed mainly in Si XI emission by EIT in $304 \AA$ A. Figure 3 of Ciaravella et al. (2002) shows LASCO $\mathrm{C} 1$ images in Fe XIV $\lambda 5302.42$ taken at different times on 1998 March 23, and displays the evolution of the magnetic configuration in the event; Figs. 8 and 9 of Ciaravella et al. (2002) reveal features of the resultant flare loop system seen in various wavelengths, respectively.

By comparing the relative locations of various important features developed in the eruptive process shown in Fig. 4 with the CME/flare magnetic configuration predicted by Lin and Forbes (2000), Ciaravella et al. (2002) suggested that the narrow and bright feature discussed above could be the region where the reconnecting current sheet between the CME and the associated flare was located. This is the first identification of the possible location of the CS via the spectroscopic approach. As we noted in a previous section, we use the term of CS in this paper in a general sense.

When studying the spectral data of the 2002 April 21 event, Innes et al. (2003a) noticed the emission of the high temperature $\left(\sim 10^{7} \mathrm{~K}\right)$ spectral line, [Fe XXI] 1354A, and SADs observed by both TRACE and SOHO/SUMER. This event was observed by LASCO and UVCS as well. It developed a fast CME $\left(\sim 2500 \mathrm{~km} \mathrm{~s}^{-1}\right)$ and an X-1.5 two-ribbon flare, and has been studied extensively (e.g., see also Innes et al. 2003b; Sheeley et al. 2004; Reeves et al. 2008a; and references therein). It is probably the first event developing a CS that was observed face-on [namely the line-of-sight (LOS) is perpendicular to the CS plane and along the two thick horizontal arrows shown in Fig. 2]. At the location where the [Fe XXI] emission was seen, the emission of low temperature spectral lines like C II did not appear. This, again, confirmed apparently plasma heating that took place as a result of magnetic reconnection in the CS above flare loops. Spectra of the reconnection outflow in the CS revealed flows of high speed up to $10^{3} \mathrm{~km} \mathrm{~s}^{-1}$ (Innes et al. 2003b).

Webb et al. (2003) investigated $26 \mathrm{CME}$ events observed in white light by the Solar Maximum Mission satellite. These events displayed magnetic disconnection features, basically rapidly evolving concave-outward bright regions behind CME fronts, and they are 


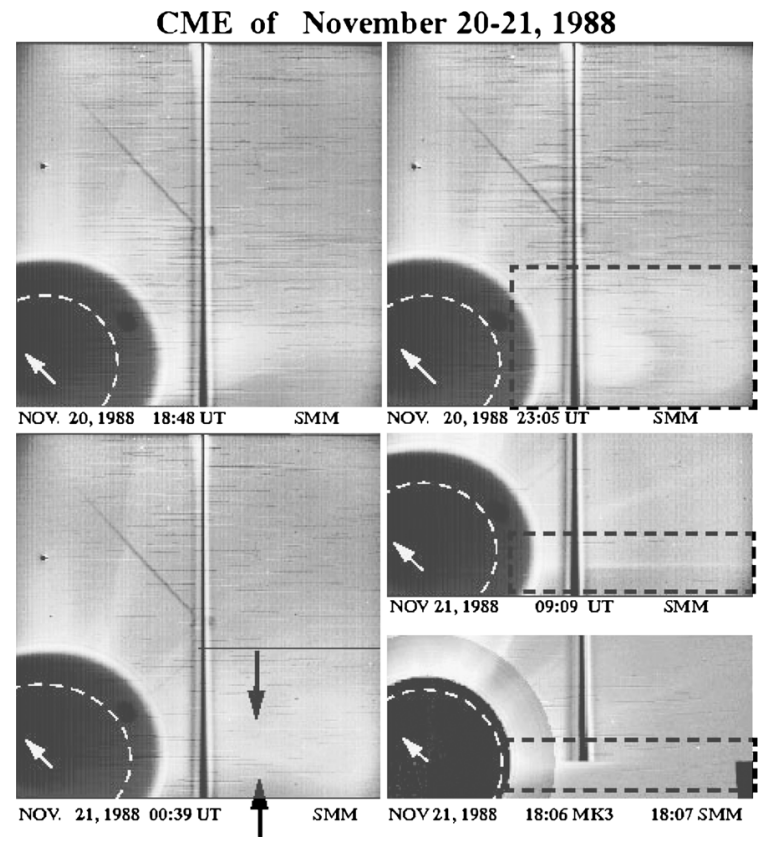

Fig. 5 Evolution of the 20-21 November 1988 SMM CME in the southwest. The pre-existing streamer swelled and brightened (20, 18:48 UT), then blew out as the CME. The CME had a broad outer loop enveloping a dark cavity, within which appeared a bright, circular core (20, 2305 UT). The core later revealed a V-shaped "back" with a stalk extending below the occulter (21, 00:39 UT). Hours later, a very bright, narrow ray suddenly appeared a few degrees south of the axis of the previous CME and CDE (21, 09:09 UT). Below this panel is a Mauna Loa Solar Observatory (MLSO) image later on this day superposed within the SMM occulting disk, showing that the ray was part of a newly formed helmet streamer. The curved dashed lines indicate the limb of the Sun, and the arrows point to solar north. The vertical and diagonal lines and the small circular feature in each panel are detector artifacts. From Webb et al. (2003)

also known as the "candidate disconnection events (CDEs)". A set of ray-like structures were observed to form several hours after these CDEs, and they sometimes were seen as well to connect to the streamers above the limb. Two examples of SMM CMEs with CDEs, which were followed by trailing, transient ray-like structures, were chosen to demonstrate the approach for recognizing the structures and identifying the current sheet. Figure 5 displays the evolution of the 20-21 November 1988 CME over the southwest limb. It was typical of the events in several aspects. The pre-existing streamer swelled and brightened, then blew out as the CME, which had a broad, outer loop enveloping a dark cavity, within which appeared a bright, circular core. So this CME possesses the typical threecomponent structure, namely bright front, dark cavity, and bright core (see Lin et al. 2004; Lin and Soon 2004; and references therein).

The bright core expanded outward, revealing a V-shaped "back" with a stalk extending below the occulter. Many hours later, a very bright and narrow ray structure suddenly appeared a few degrees south of the axis of the previous CME and CDE. As shown in the bottom right panels, the ray was also visible later in the day in the images obtained by the Mauna Loa Solar Observatory's K-Coronameter, in which it appeared as an extension of a newly formed helmet streamer. After having compared with observations regarding emission and structure features of the CS (see Ciaravella et al. 2002), and with theoretical expectations of the CS dynamic behaviors given by both analytic solution (see Lin and Forbes 2000; 
Lin 2002) as well as numerical experiments (see, for example, Linker et al. 2003), Webb et al. (2003) regarded this kind of ray-like structures as evidence of the current sheet developed following a CME. This is the first time that a survey of ray-like structures has been performed and the results have been compared with CME models, and is also the first time that the CS was recognized by studying the white-light data. With the success of SDO, the CS associated structures right above flare loops have as well been observed in the AIA $131 \AA$ and $94 \AA$ channels in exquisite detail both as rays when viewed edge on (Reeves and Golub 2011), and as fans when viewed face on (Warren et al. 2011; Savage et al. 2012a; McKenzie 2013).

Raymond et al. (2003) studied the 2002 April 21 event and another two events occurring on July 23 and August 24, 2002, respectively, that all produced X-class (>1.5) flares with associated fast CMEs at speeds of $1700-2500 \mathrm{~km} \mathrm{~s}^{-1}$. These events occurred in active regions targeted by the Max Millennium Campaign, and were well observed by many instruments both in space and on the ground. The UVCS slit was placed where both CMEs and the CSs behind successively passed through. The UVCS data manifested clear spectral signatures in terms of very rapid disruption of the pre-CME helmet streamer suggesting that all the eruptions were initiated in the magnetic structures below helmet streamers, very high Doppler shifts that indicating high speed motion component along the line-of-sight (LOS), and high-temperature plasma visible in the [Fe XVIII] emission line implying intensive heating taking place during the events.

Raymond et al. (2003) noticed that all three events displayed transient brightennings in the [Fe XVIII] line and little or none of the cool gas that usually dominates UVCS spectra of CMEs, and that the violent disruption of the pre-CME streamer was manifested by splitting of the low temperature $\left(2.9 \times 10^{5} \mathrm{~K}\right) \mathrm{O}$ VI line profiles into strongly red and blue shifted components, and by the rapid growth of the region of split profiles along the UVCS slit. Following splitting of the O VI line was transient brightening in the [Fe XVIII] line, placing it well inside the CME front. Consulting Fig. 2, we noticed that the hot plasma created in the current sheet by reconnection could enter the outer shell of the CME bubble (see the red component around the flux rope), and freezing of plasma to the magnetic field confines the hot plasma to the shell. Therefore, the plasma in the other components is cooler than those in the outer shell, which would lead to the transient brightening in the [Fe XVIII] line as the CME passes UVCS slit quickly. These results depicted the dynamic scenario of the fast CMEs via spectral signatures, which was also theoretically described by Lin and Soon (2004) and Lin et al. (2004). Raymond et al. (2003) pointed out that the features seen in [Fe XVIII] line were quite likely the post-CME CS.

Bemporad et al. (2006) studied an event on 2002 November 26, at the time of a SOHOUlysses quadrature campaign, which made their work unique because the SOHO-Ulysses data set allowed them to analyze a current sheet structure from its lowest coronal levels out to its in situ properties. The event occurred over the northwest limb and produced a CME with speed at the front increasing from 90 to $420 \mathrm{~km} \mathrm{~s}^{-1}$ and a flare that the Geostationary Operational Environmental Satellite (GOES) system did not class because the main part of the flare was behind the limb, but the growth of the post-CME loop system could be clearly seen. The orientation of the disrupting magnetic configuration resulted in a CS between the flare loop and the CME that had been continuously observed face-on for two days, which made it relatively easy to investigate its temporal behavior. The post-flare loop system continued to be observed in EIT Fe XII and He II for more than 24 hours, and its heights gradually rose from 0.06 to $0.34 \mathrm{R}_{\odot}$ above the solar surface (see also Fig. 2 of Bemporad et al. 2006, which displayed the images of the flare loop system at different times after the associated CME, as well as the change in the loop heights versus time). However, the typical 
cusp feature at the end of the CS did not appear in the EIT images, and it did not appear in the LASCO images, either.

About 1.5 hours after the CME left the field-of-view (FOV) of EIT, UVCS started to take spectral data with its slit located at a heliodistance of $1.7 \mathrm{R}_{\odot}$, covering emissions from both cool and hot plasmas. The emission of [Fe XVIII] indicated the high temperature of plasma due to magnetic reconnection in progress, and its eventual fading implied that the reconnection process was slowing down or stagnating because the free magnetic energy in the system ran out. At the time of the quadrature, Ulysses was directly above the location of the CME and intercepted the ejecta. High ionization state Fe ions (up to Fe XVII) were detected by the Ulysses SWICS throughout the magnetic cloud associated with the CME, compared to the normal charge state of Fe in the solar wind being about 10.

Poletto et al. (2004) extrapolated Ulysses measurements back to the Sun using the in situ measurement of the flow speed of the solar wind plasma, which gave a rough estimate of the origin time for the plasma, and yielded a positive identification of the plasma resulting from the 2002 November $26 \mathrm{CME}$ by comparing features in the solar wind with phenomena directed toward Ulysses in the corona. Therefore, Bemporad et al. (2006) concluded that a relation of the ICME detected by Ulyssees to the CME observed near the Sun by several other instruments could be set up, and the model shown in Fig. 2 fits the scenario manifested by this event very well. Bemporad (2008) further strengthened this conclusion.

To our knowledge, this event is the only one so far whose eruption and consequences were observed (detected) by both remote sensing and in situ instruments. Detecting the high ionization state Fe ions by the Ulysses SWICS implies that magnetic reconnection was still occurring in a very elongated CS a couple of days after the initiation of the event, that the CS was still connecting to the rear part of the CME, and the reconnected hot plasma was continuously sent into the CME bubble as suggested by the theoretical scenarios described by Fig. 2 (e.g., see also detailed discussions of Lin et al. 2004 and Lin and Soon 2004). It is not easy to determine where the CS terminates at the far end although we know that the distance between the CME core and the CS end depends on the rate of magnetic reconnection in the CS and the local Alfvén speed around the CS (e.g., see also Forbes and Lin 2000; Lin and Forbes 2000; Lin 2002). In fact, in the Petschek reconnection picture, there may no real distinction, or there may be a shock (e.g., see Shiota et al. 2005). This leaves an open question to our works in the future.

As for the lower tip of a CS and the associated typical cusp structure below it, on the other hand, there are many observations and studies that have been reported (see discussions in Sect. 3.2 below for details). Usually, these observations were made not far from the surface of the Sun because the cusp structure above the flare loop system cannot get very high in the corona. Forbes and Lin (2000), Lin and Forbes (2000), and Lin (2002) pointed out that the kinematic behavior of the lower tip of CS, namely the cusp point over the flare loop system, is determined by the rate of magnetic reconnection in the CS itself. Both theoretical calculations (Lin 2002) and numerical experiments (Linker et al. 2003) indicated that the highest altitude to which the CS lower tip could reach in the coronal environment within the typical life time of the major flare (tens of hours) is around $2.5 \times 10^{5} \mathrm{~km}\left(0.36 \mathrm{R}_{\odot}\right)$.

Observations suggested that this value varies from case to case, but is not very different from the theoretical value. We list results for this value deduced from observations of the typical events in various eras in Table 2 for comparison. We notice that the cusp structure above the flare loop system are usually observed below $0.5 \mathrm{R}_{\odot}$ from the solar surface. This height is obviously below the edges of the occulting disks of the LASCO/C2 and C3 that are located at $1 \mathrm{R}_{\odot}$ and $2 \mathrm{R}_{\odot}$ above the solar surface, respectively. Therefore, the cusp region in a flare event cannot appear in the FOV of either $\mathrm{C} 2$ or $\mathrm{C} 3$. LASCO $\mathrm{C} 1$ might be able to 
Table 2 Heights of cusp structures observed in various events

\begin{tabular}{llll}
\hline Event date & Cusp heights $\left(\mathrm{R}_{\odot}\right)$ & Instruments & Reference \\
\hline 21 Feb 1992 & 0.54 & Yohkoh/SXT & Forbes and Acton (1996) \\
06 Nov 1980 & 0.26 & SMM & Švestka (1996) \\
20 Jan 1999 & 0.14 & Yohkoh/SXT & McKenzie and Hudson (1999) \\
20 Apr 1998 & 0.22 & Yohkoh/SXT & McKenzie (2000) \\
23 Apr 1998 & 0.40 & Yohkoh/SXT & McKenzie (2000) \\
23 Mar 1998 & 0.25 & SOHO/EIT & Ciaravella et al. (2002) \\
08 Jan 2002 & 0.40 & SOHO/EIT & Ko et al. (2003) \\
23 Jul 2002 & 0.10 & TRACE & Asai et al. (2004) \\
18 Nov 2003 & 0.25 & SOHO/EIT & Lin et al. (2005) \\
26 Nov 2002 & 0.35 & SOHO/EIT & Bemporad et al. (2006) \\
21 Apr 2002 & 0.17 & TRACE & Reeves et al. (2008a) \\
02 May 2007 & 0.20 & Hinode/XRT & Reeves et al. (2008b) \\
09 Apr 2008 & 0.25 & Hinode/XRT & Savage et al. (2010) \\
22 Oct 2011 & 0.22 & SDO/AIA & Savage et al. (2012a) \\
19 Jul 2012 & 0.32 & SDO/AIA & Liu et al. (2013) \\
03 Nov 2003 & 0.18 & NRH & Aurass et al. (2013) \\
19 Jan 2012 & 0.18 & SDO/AIA & Innes et al. (2014) \\
07 Sep 2005 & 0.30 & MLSO/MK4 & Ling et al. (2014) \\
\hline
\end{tabular}

observe the structure, but it failed to work soon after the launch of SOHO. Hence, to our knowledge, no study of the CS lower tip on the basis of the LASCO observations has ever been reported.

The SXR emission above the solar limb at altitudes ranging from 0.1 to $0.3 \mathrm{R}_{\odot}$ from the solar surface during the 2002 November 26 event was also observed by RHESSI (e.g., see Saint-Hilaire et al. 2009). This high-altitude coronal X-ray source (HACXS) lasted for 12 hours, and the corresponding temperature peaked around $(1.0 \sim 1.1) \times 10^{7} \mathrm{~K}$ with its emission measure increasing throughout this time interval. It was also observed to progress gradually outward at speed up to $14 \mathrm{~km} \mathrm{~s}^{-1}$. Saint-Hilaire et al. (2009) noticed that the emission was dominated by the thermal component and lacked a contribution from nonthermal hard X-rays (HXRs). They interpreted the hot plasma as the signature of the material inside a CS in the wake of the CME, and suggested that heating occurring inside the CS could be directly due to the heat conduction from the HACXS and reconnection itself, and that the energetic particles were unlikely to account for the plasma heating. However, the information they collected could not help determine whether the CS and the HACXS were co-located in space. On the other hand, locations and temporal behavior of the HACXS and the EUV loop systems they observed supported the picture that the reconnection site above the loop top continuously moves upward, heating the local plasma to the temperatures of X-ray emission, before it cooled down and was later seen in the EUV. This implied that the EUV loops trailed the X-ray source in both space and time, and that the higher energies were located higher than the lower energies (see also Gallagher 2002), constituting the standard Kopp-Pneuman two-ribbon flare configuration.

Susino et al. (2013) studied EUV/UV data from UVCS, HXR data from RHESSI, and SXR data from GOES of the 2004 July 28 event that developed a partial halo CME with maximum velocity of $\sim 754 \mathrm{~km} \mathrm{~s}^{-1}$ in the online LASCO CME catalog (Gopalswamy et 
al. 2009) and a small flare. A narrow emission feature in [Fe XVIII] $\lambda 974 \AA$ A was observed to stand for about 2.5 days by UVCS after the CME crossed the UVCS slit. Analyzing the UVCS data indicated apparent non-thermal broadening due to the turbulent motion inside the CS, and a large density gradient across the CS region, and studying the X-ray data showed that SXR and HXR sources evolved differently from the EUV/UV sources. They concluded that the reconnection process was very likely to be turbulent in the CS, but reconnection occurring above the post-eruption arcades was unlikely to be directly responsible for the high-temperature plasma sampled higher up by UVCS, instead the HXR could be the direct source of high temperature plasma sampled higher up by UVCS as suggested by Saint-Hilaire et al. (2009).

However, we also need to point out here that, according to the standard model of the tworibbon flare (see Forbes and Acton 1996; Priest and Forbes 2002; and references therein), the HXR source on the top of the post-flare loop is basically created by the collision of the energetic particles and the downward reconnection outflow from the CS with the closed magnetic field lines where flare loops are believed to lie on (see also the lower part of Fig. 2). This is the so-called Masuda scenario of solar flare (e.g., Masuda et al. 1994; Forbes and Acton 1996; Krucker et al. 2008). The lower part of Fig. 2, which is a duplicate of Fig. 1 of Forbes and Acton (1996), describes clearly where the interaction takes place. As we can see from this figure, the interaction region is already detached from the CS although it is still located on the top of the flare loop system, and the hot plasma responsible for the HXR emission is confined in the closed field lines that are not magnetically connected to the CS (see also Fig. 4 of Lin 2004 that describes the evolution of magnetic field lines when the flare loop system is produced by reconnection). Therefore, the thermal energy of the HXR source region within flare loops is not able to propagate to the reconnection region via thermal conduction, and thus cannot account for observational results obtained by UVCS for the CS. Studies of Caspi and Lin (2010) clearly showed that the super-hot $(\sim 45 \mathrm{MK})$ plasma was observed high in the corona, co-locating with a non-thermal coronal HXR source, before the HXR emission could be detected in the lower altitudes. So Caspi and Lin (2010) concluded that this super-hot plasma originated in the corona.

In the framework of Petschek-type reconnection (Petschek 1964), on the other hand, the diffusion region is tiny, and most of the energy dissipation occurs in the shocks that bound the exhaust flow, which includes an upward component and a downward component (see also Vršnak et al. 2009; Ko et al. 2010). Numerical experiments of Mei et al. (2012) showed that a CS in the CME/flare process actually possesses complex structures, in which three types of magnetic reconnection: Sweet-Parker, Petschek, and turbulence, could even take place simultaneously. So UVCS is observing the reconnection region, though much of the emitting gas might not be heated at the position observed, but somewhere between this position and the X-line. As we mentioned earlier in this work as well as in our previous work (e.g., see Lin et al. 2009), the reconnection region discussed here is, in fact, the CS in a more general sense.

Studies of Aurass et al. (2009) demonstrated as well heating of the plasma inside the CS by magnetic reconnection during the eruptive event occurring on May 17, 2002. This event was observed to take place on the east limb by many instruments. It produced an M-1.5 flare (see also Karlický et al. 2004) and a CME at an average speed of $665 \mathrm{~km} \mathrm{~s}^{-1}$ according to the SOHO/LASCO CME Catalog. ${ }^{1}$ LASCO/C2 images enhanced by the wavelet method showed a bright "stem" feature behind the CME core at the position where a CS is presumably located according to the Lin and Forbes (2000) model. The EUV spectral data from

\footnotetext{
${ }^{1}$ http://cdaw.gsfc.nasa.gov/CME_list/.
} 
UVCS allowed them to deduce the plasma temperature and density of the CS, and the results of Aurass et al. (2009) indicated that the CS extended from, at least, the heliocentrical distance of 1.5 to $2.1 \mathrm{R}_{\odot}$. Distributions of brightness in O VI and in Si XII images along the UVCS slit showed that a dimming in the O VI image is co-located with a brightening in the Si XII image in space, and analyzing the intensity ratio of these two spectral lines in that region gave a temperature up to $5 \times 10^{6} \mathrm{~K}$, which indicated intensive heating occurring inside the CS.

Radio data obtained by AIP and Ondřejov radiospectrographs showed simultaneously several groups of drifting pulsating structures (DPSs) in both high $(>2 \mathrm{GHz}$ ) and low $(40-170 \mathrm{MHz})$ frequencies, and type III radio bursts in the low frequency. The DPS at low frequency and the type III burst took place during the decay of HXR emission observed by RHESSI. Karlický et al. (2004) noticed that the HXR source is located where the rising EIT loops appeared in the impulsive phase of the associated flare. The frequency range of the type III radio burst and the low frequency DPSs indicated the energetic particles were mainly accelerated in the middle and high coronal region where the plasma density corresponds to the frequency range between 40 and $170 \mathrm{MHz}$. Aurass et al. (2009) and Karlický et al. (2004) did not provide the information of the location of this region in space, but we can estimate the location and the extension of this region approximately by relating the above frequencies to the plasma density if the distribution of the plasma density in the corona is given.

Using the empirical model of Sittler and Guhathakurta (1999) for distributions of the plasma density in the corona, we deduced that the above frequencies correspond to a height range in the corona from the heliocentrical distance of 1.43 to $2.19 \mathrm{R}_{\odot}$ where energetic particles responsible for the DPS and the type III bursts were accelerated. This is consistent with that of the CS deduced from the UVCS data. So we conclude that those energetic particles were accelerated inside the CS by magnetic reconnection, which also accounts for heating plasma as indicated by the anti-correlation of the Si XII emission to the O VI emission in the CS (see also discussions by Aurass et al. 2009), and that meter wave radio imaging and meter wave radio spectroscopy provide us another access to look into the reconnection region based on the plasma emission due to energetic electrons accelerated in the CS (see discussions below as well).

Observations discussed above were mainly focusing on the CS below the flux rope and roughly perpendicular to the solar surface. Recently, radio observations, together with observations in the other wavelengths, suggested the formation and development of a CS parallel to the solar surface during the eruption (e.g., see Aurass et al. 2013; Aurass 2014). Aurass et al. (2013) studied an eruptive event taking place on November 3 , 2003, which developed a major flare of X-3.9 class and a fast CME, focused on the impulsive phase, and looked into key details of the complementary radio data of high time cadence.

They studied the radio data from the Leibniz-Institut für Astrophysik Potsdam (AIP), the simultaneous radio images were from the Nançay Multi-frequency Radio Heliograph (NRH), and the single frequency polarimeter data from the Trieste Astronomical Observatory (OAT). The radio emission was apparently non-thermal plasma radiation with a complex structure in both frequency and time. Veronig et al. (2006) studied the motion of the HXR flare loops and noticed the shrinkage of the loop system during the impulsive phase of the event, and the radio data analyzed by Aurass et al. (2013) were obtained right in the time interval when the top of the flare loop system reached its lowest location in height (see also Fig. 2 of Aurass et al. 2013, which displays the impulsive phase synopsis of the event with the AIP dynamic spectrum, radio and X-ray data time line, as well as RHESSI HXR counts). 
Two stationary radio sources at meter wavelengths were observed by Aurass et al. (2013) radially aligned at 0.18 and $0.4 \mathrm{R}_{\odot}$ from the solar surface above the active region and the HXR sources, respectively. The lower source was believed to be the outflow region just above the X-line in the CS, while the upper one was related to that occurring in the CS above the top of the expanding arcade, and the radio emission at lower frequencies surrounded the upper source at the expected locations as displayed in the breakout scenario (see also Fig. 3). Aurass et al. (2013) thus concluded that the upper radio source was the most compelling evidence to date for the onset of breakout reconnection during an eruption. The height stationarity of the breakout sources and their dynamic radio spectrum discriminate them from propagating disturbances. Timing and location arguments revealed for the first time that both the earlier described above the flare loop top HXR source and the lower radio source were emission from the reconnection outflow above the vertical flare (tail) CS.

Furthermore, Aurass (2014) investigated the radio emission of the same event in the late gradual phase. In addition to the data from AIP, NRH, and OAT, the data from RHESSI, $\mathrm{SOHO} / \mathrm{LASCO}$, and SOHO/EIT were also used. The results indicated that the late gradual phase started about 6 min and $15 \mathrm{~s}$ after the end of the impulsive phase, and that the late phase consisted of two stages. At the first stage, a radio emission re-occurred at the breakout reconnection site, the maximum of this source shifted northeast in the following several mins, then another source appeared aligned with the motion azimuthally. In this process, the breakout reconnection site decayed and then re-appeared. Aurass (2014) found that the projected speed of the motion is about $1200 \mathrm{~km} \mathrm{~s}^{-1}$, compared with $1100 \mathrm{~km} \mathrm{~s}^{-1}$ deduced by Vršnak et al. (2006) from the drift rate of the type II radio burst observed in the same event. Aurass (2014) also noticed that the disturbance caused by these motions to the corona was also observed by SOHO/EIT in $171 \AA$, and that the emission at high frequency (236.6 MHz) occurred at outermost location from the Sun, and that at low frequency $(150 \mathrm{MHz})$ was closer to the Sun. This means that the local density distribution behaves in a way opposite to the normal coronal density distribution.

The reappearance of the breakout reconnection site marked the beginning of the stage 2 , in which radio emission sources at frequencies lower than $236.6 \mathrm{MHz}$ were seen to move at velocity $\sim 400 \mathrm{~km} \mathrm{~s}^{-1}$, apparently lower than the speed observed earlier. The feature moving at this speed was also observed by SOHO/LASCO C3 behind the CME four hours later. At this stage, the radio sources at higher frequencies appeared right above the active region with growing intensity. This scenario was explained as the process in which the CME bubble went through the breakout CS above the disrupting magnetic configuration, the global coronal magnetic field reconnected with the field around the tail CS that connected CME to the associated flare, and the second stage is the phase of the eruption in which the coronal breakout relaxation took place after the CME had left. Aurass (2014) believed that this was the first report of the coronal breakout recovery, and that their interpretation of the observation confirmed some predictions given by the numerical experiments of Lynch et al. (2008).

\section{Plasma Flows Inside and Around the Reconnection Current Sheet in the Eruption}

In addition to high temperatures inside the current sheet, there exist other important observational features. As shown by Fig. 2, two reconnection outflows move along the current sheet in opposite directions. The sunward (downward) flow contributes to the formation of flare loops, and the anti-sunward (upward) flow governs the evolution of the structures of the 
CME bubble (see also Lin and Soon 2004; Lin et al. 2004; Mei et al. 2012 for more details). Therefore, observations of the reconnection outflows above the flare loop system and below the associated CME could be expected. In addition, Fig. 2 also indicates the reconnection inflow near the CS, which could be considered as another observational consequence of the model. In this section, we will discuss the reconnection inflows, the sunward (downward) outflows, the anti-sunward (upward) outflows, bidirectional outflows, and finally outflows observed during laser driven reconnection.

\subsection{The Reconnection Inflow Near the Current Sheet}

The first aspect of reconnection that we will discuss is observations of inflowing plasma into the CS region. As reconnection proceeds, the reconnection inflow continuously brings the magnetized plasma into the CS, and the magnetic energy is converted into heat and kinetic energy of the plasma and energetic particles. The amount of the magnetic energy entering the CS is balanced by the amount of energy dissipated by reconnection, otherwise either the magnetic field and the plasma pile up outside the CS if the dissipation rate is slower than the input rate, or reconnection ceases if the dissipation is faster than the input. Thus a fast reconnection process requires a fast reconnection inflow. Therefore, the reconnection inflow speed $v_{i}$ is a proxy to describe the rate of energy conversion in the CS. Usually, we use dimensionless parameters to describe the processes/phenomena of interest in order to deduce the related scaling laws, and $M_{A}=v_{i} / v_{A}$ is often used as the dimensionless measure of $v_{i}$, where $v_{A}$ is the local Alfvén speed in the same region. In the magnetized plasma, the flow speed of plasma is lower than $v_{A}$, and so is $v_{i}$, in the absence of the fast mode shock. The advantage of using $M_{A}$ is that it ranges from 0 to 1 , and can be easily related to other parameters via various scaling laws. Hence, $M_{A}$ is frequently used in studies of magnetic reconnection. Usually, fast reconnection has $M_{A}$ of around $0.01 \sim 0.1$ (see discussions of Petschek 1964; Priest and Forbes 2000).

As we noted before, identifying the current sheet is not trivial, so it is difficult to measure either $v_{i}$ or $v_{A}$ near the current sheet. To our knowledge, Yokoyama et al. (2001) performed the tentative measurement of $v_{i}$, and then $M_{A}$ for the first time. They studied images of the cusp region above the flare loop system of the event of 1999 March 18 obtained by SOHO/EIT and Yohkoh/SXT, and noticed a well recognized cusp structure and a plasma blob in the SXT image (or movie) and a blob-like cavity in the EIT $195 \AA$ Amage (or movie). The cavity was dark in EUV wavelength and bright in SXR, which suggested that the temperature of the plasma in the cavity was about $4 \times 10^{6} \mathrm{~K}$. As the cavity moved away from the solar limb, an X-like structure appeared behind it, and some thin filament-like structures were seen to merge together from the two sides of the X-like structure. This merging pattern was identified as the trajectories of the inflow near the reconnection region. A speed of the reconnection inflow between 1.0 and $4.7 \mathrm{~km} \mathrm{~s}^{-1}$ was deduced by measuring the speed of merging motion seen in EIT $195 \AA$ images. A disadvantage of this measurement exists though since the structure seen in EIT $195 \AA$ images is usually diffuse, so it is difficult to determine its velocity. Chen et al. (2004) re-analyzed the data of Yokoyama and Shibata (2001), and realized that the merging motion toward the X-point was, in fact, caused by the change in the location of the reconnection region, and was not the true inflow.

In the spirit of works by Yokoyama et al. (2001) and Ko et al. (2003), Lin et al. (2005) looked into various aspects of an event taking place on November 18, 2003. The event started with a rapid expansion of a few magnetic arcades located over the east limb of the Sun and developed an energetic partial-halo CME, a long CS, and a group of bright flare loops in the wake of the CME. It was observed by several instruments, both in space and 
Fig. 6 Composite

C2-UVCS-EIT image for the 2003 November 18 event. The C2 image was taken at 10:26 UT and has been enhanced by the wavelet technique in order to emphasize the morphological features at various positions of the CME, the EIT $195 \AA$ A image was taken at 10:14 UT, the left UVCS image was made by stacking five UVCS exposures in $\mathrm{Ly} \alpha$ that were taken at an altitude of $1.70 \mathrm{R}_{\odot}$ and cover the time interval from 10:04 UT (right edge) to 10:14 UT (left edge), and the right UVCS image was made by stacking four UVCS exposures in Ly $\alpha$ that were taken at an altitude of $1.52 \mathrm{R}_{\odot}$ and cover the time interval from 10:17 UT (right edge) to 10:23 UT (left edge).

From Lin et al. (2005)

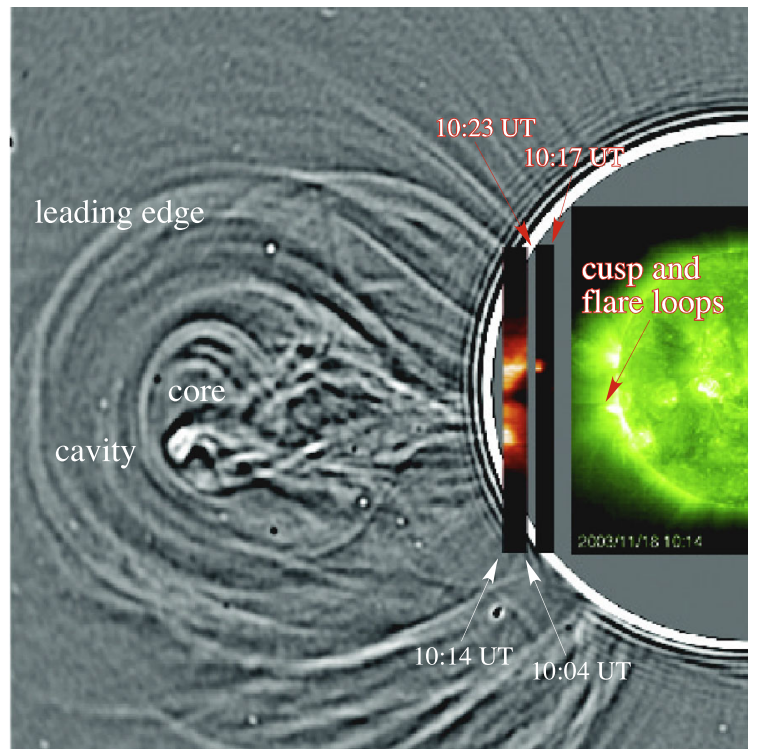

on the ground, including EIT, UVCS, and LASCO on board the SOHO, the RHESSI, and the MLSO MK-4 coronameter. Lin et al. (2005) combined the data from these instruments to investigate various properties of the eruptive process, including those around the CS. The maximum velocities of the CME leading edge and the core were 1939 and $1484 \mathrm{~km} \mathrm{~s}^{-1}$, respectively. The average reconnection inflow velocities near the current sheet over different time intervals ranged from 8 to $85 \mathrm{~km} \mathrm{~s}^{-1}$, and the average outflow velocities ranged from 460 to $1075 \mathrm{~km} \mathrm{~s}^{-1}$, which brought $M_{A}$ to the range from 0.007 to 0.18 . According to discussions above, the values of $M_{A}$ in this range indicate the fast reconnection, which was expected since the event studied produced a fast CME associated with a group of post-flare loops. In the work of Lin et al. (2005), the inflow speed was determined directly according to the change in Ly $\alpha$ images of the UVCS slit that described the pattern of the reconnection inflow in a simple and straightforward fashion, so the problem that Chen et al. (2004) found with Yokoyama et al. (2001) was avoided.

As for the local Alfvén speed $v_{A}$ in the inflow region, Lin et al. (2005) took an approximation to first order that the magnetic pressure, $B_{i}^{2} / 8 \pi$, in the inflow region balances the dynamic pressure, $\rho_{\text {out }} v_{\text {out }}^{2} / 2$, in the outflow region as the magnetic reconnection takes place continuously. Here $v_{\text {out }}$ and $\rho_{\text {out }}$ are the plasma density and velocity in the outflow region, respectively. To the first order of approximation again, $\rho_{\text {out }}=\rho_{i}$, where $\rho_{i}$ is the plasma density in the inflow region. Therefore, equating the magnetic pressure in the inflow region to the dynamic pressure in the outflow region gives, $v_{\text {out }}=v_{A}=B_{i} / \sqrt{4 \pi \rho_{i}}$, which is the Alfvén speed in the magnetic reconnection inflow region. This means that the local Alfvén speed $v_{A}$ in the inflow region can be approximated with the reconnection outflow speed $v_{\text {out }}$ to the first order.

In principle, the approach taken by Lin et al. (2005) to determining the inflow speed near the CS was not different from that taken by Yokoyama et al. (2001) and then by Chen et al. (2004), as well as by the other authors who focused on the similar topic. The only difference lies on the manifestation of the same process observed by different instruments and/or at different wavelengths, and such manifestations vary from case to case as well. When performing the measurement, we chose those features of the motion pattern that could 
be recognized easily. In the work of Yokoyama et al. (2001) and Chen et al. (2004), EIT data was used to determine the inflow speed because the motion of the relevant structures seen in EIT $195 \AA$ was the most apparent manifestation of the reconnection inflow; and in the work of Lin et al. (2005), on the other hand, the Ly $\alpha$ data were used because the manifestation of interest could be seen most clearly in this wavelength although the same manifestation was also shown in EIT $195 \AA$ (see Fig. 2 of Lin et al. 2005 that displays a set of EIT $195 \AA$ images showing the process that is characterized by the magnetic arcades' disruption, the driven magnetic reconnection, and the formation of flare loop system with a cusp structure on the top).

It could be seen clearly in the EIT $195 \AA$ movies that the eruption commenced with severe stretching of the closed magnetic arcade followed by approach of the two legs of the stretched arcade to one another, and a group of flare loops topped with a cusp area subsequently developed at a lower region (see Fig. 2 of Lin et al. 2005 for details). Figure 6 displays a composite of LASCO/C2, UVCS/Ly $\alpha$ slit, and EIT $195 \AA ̊$ images taken roughly at the same time in this process. If we ignore the error in co-locating the images due to the fact that these images were not taken exactly at the same time, Fig. 6 displays the main aspects of the magnetic configuration that may appear in the major eruption, which usually produces both flare and CME (cf. Fig. 2). The image of the UVCS slit is actually a composite of 5 filtergrams in Ly $\alpha$ taken at 5 times successively. The distribution of the brightness along the slit indicates the change in the intensity of $\operatorname{Ly} \alpha$ emission in that direction, and the horizontal scale of the UVCS images does not represent the extension in space, but in time.

Therefore, from the right to the left of the UVCS slit images in Ly $\alpha$, changes in the shape of both the dark gap and bright spots indicate the decrease in the gap width with time (see also Fig. 11a of Lin et al. 2005, which showed five Ly $\alpha$ intensity profiles along the UVCS slit taken at $1.70 \mathrm{R}_{\odot}$ from 10:04 to 10:14 UT during the event. The dip in each profile is suggestive of the dark gap shown in Fig. 6). Comparing with the images taken at the corresponding times that display motions of the eruptive arcade legs (see Fig. 2 of Lin et al. 2005), we can see that the dark gap coincided with the motions of the two legs of the eruptive arcades in both space and time, and that the gradual disappearance of the gap could be ascribed to the reconnection inflow near the CS. Measuring the gap widths at different times allows us to look into the evolution in this region. We plot the width of the gap at five different times in Fig. 7, and obtain correspondingly four speeds of the gap shrinkage as indicated in the figure. Since this event occurred on the limb and the orientation of the disrupted magnetic configuration led to a CS just along LOS (i.e., edge-on) as illustrated in Fig. 2, these speeds were approximately the speed of the reconnection inflow near the CS.

We note here that the dark gap seen in Ly $\alpha$ image could be due to either high temperature or high speed of plasma flow perpendicular to LOS or both in that region. At the location shown in Fig. 6, on one hand, the emission of $\operatorname{Ly} \alpha$ comes from the resonant scattering of the photospheric emission by the neutral hydrogen atoms (H I), whose intensity is proportional to the density of H I. So Ly $\alpha$ emission may disappear if there are few H I atoms due to high temperature. On the other hand, the intensity of $\operatorname{Ly} \alpha$ emission also depends on the speed of $\mathrm{H}$ I perpendicular to LOS. Usually, if this speed exceeds $370 \mathrm{~km} \mathrm{~s}^{-1}$, the Ly $\alpha$ scattering profile is Doppler shifted away from the chromospheric emission profile, and the emission disappears in Ly $\alpha$. This phenomenon is known as the Doppler dimming (e.g., see Noci et al. 1987 and references therein). Generally, the Doppler dimming becomes apparent as the speed of $\mathrm{H}$ I atoms exceeds $200 \mathrm{~km} \mathrm{~s}^{-1}$.

Therefore, the bright spots in the image of the UVCS slit corresponded to the region where the H I density is high, the LOS speed is low (apparently $<370 \mathrm{~km} \mathrm{~s}^{-1}$ ), and temperature is in the range of $(0.7 \sim 3) \times 10^{5} \mathrm{~K}$. The dark gap between the two spots resulted 
Fig. 7 Width variations of the Ly $\alpha$ gap on the UVCS slit image for the 2003 November 18 event as seen in Fig. 6 versus time and the corresponding speeds of the reconnection inflow, $v_{i}$ near the CS (see also Figs. 5 and 11a of Lin et al. 2005). From Lin et al. (2007)

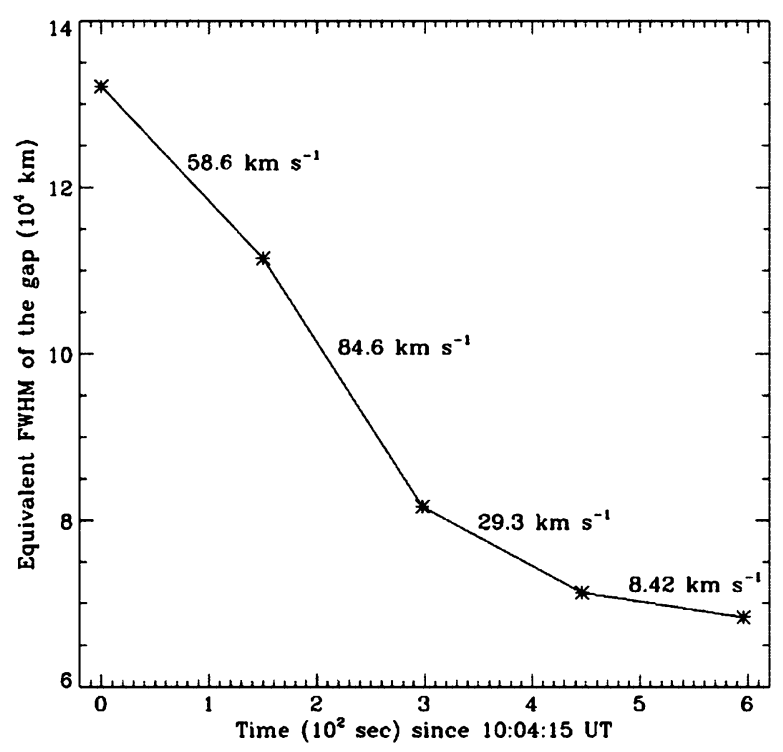

from either low density or high speed of H I atoms, or both. A low density of H I atoms means that the temperature is so high that $\mathrm{H} \mathrm{I}$ atoms have mostly ionized. Further investigation suggested that the temperature in that area may reach up to $6.7 \times 10^{6} \mathrm{~K}$, but it is also quite possible that the plasma speed is high there (see also discussions of Lin et al. 2005). No matter what happens, it always implies that the dark gap embeds the reconnecting CS connecting the CME to the associated flare. In other words, the dark Ly $\alpha$ gap marked the reconnection outflow that could result from the Petschek-type reconnection. Because the reconnection inflow and outflow regions are in close proximity to each other, the change in the size of the outflow region indicated by the decreasing width of the gap must be related to the reconnection inflow near the sheet. In another word, the decreasing width of the Ly $\alpha$ gap suggests squeezing of the outflow region, and indicates the reconnection inflow as well. We shall see later that the asymptotic behavior of the curve in Fig. 7 reveals more important information on the reconnection current sheet.

One more issue related to the work of Yokoyama et al. (2001) needs extra attention. They use $M_{A}=v_{i} / v_{A}$ to calculate $M_{A}$, but it is usually impossible to measure $v_{A}$ directly in reality. So they estimated it through an indirect approach from the soft X-ray emission measure by assuming that all the magnetic energy released during the eruption went to heating via reconnection, and that the depth $L$ of the source region along the LOS was on the same order of flare loop length, so that the source region of the SXR emission fills the whole volume of $L^{3}$ when calculating the electron density. These two assumptions introduce some uncertainty in their results: The first assumption obviously causes the magnetic field near the reconnection site to be underestimated because much (perhaps as much as half) of the released energy goes to kinetic energy of the reconnected plasma flows, and the second assumption leads the total thermal energy to being overestimated because the source region of soft X-ray could never fill the whole volume of $L^{3}$.

To avoid these uncertainties, Ko et al. (2003) and Lin et al. (2005) estimated the local Alfvén speed around the reconnection region by measuring the speed of the reconnection outflow directly. The standard theory of magnetic reconnection indicates that these two speeds are equal to one another (Priest and Forbes 2000). Because the current sheet developed by the event on November 18, 2003 was also observed edge-on, the features of the 

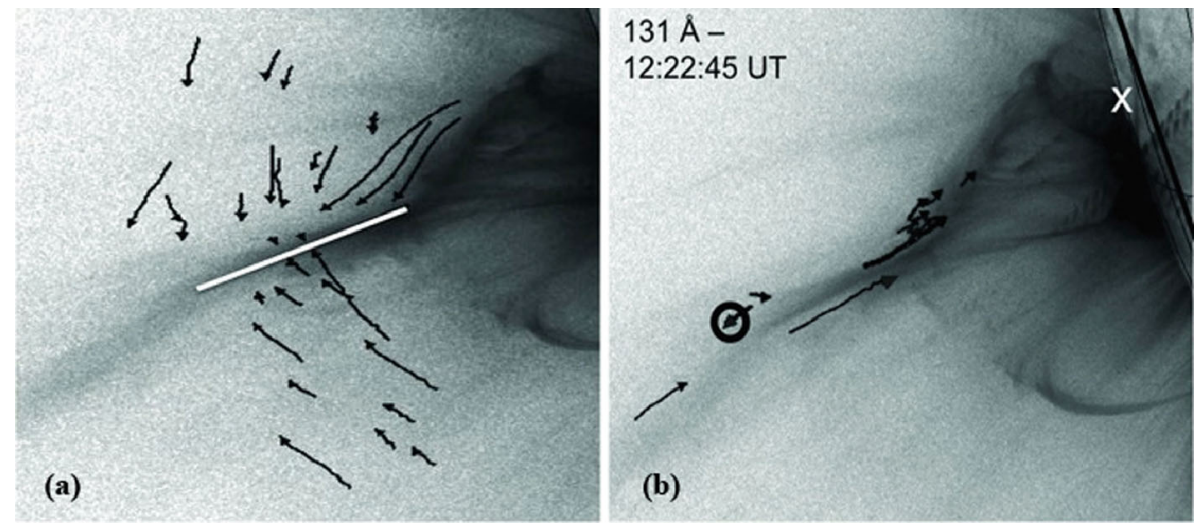

Fig. 8 (a) Composite image showing the inflow tracks from all four selected AIA filters (131 A, $211 \AA$, $193 \AA$, and $171 \AA$ ) overlaid onto a $131 \AA$ image (see also Fig. 9 of Savage et al. 2012b). (b) The sunward reconnection outflow tracks seen in $131 \AA$, with an anti-sunward flow track highlighted by a circle. The white " $\mathrm{X}$ " marks the footpoint of the magnetic configuration used to determine outflow heights. From Savage et al. (2012b)

reconnection outflow could be seen easily. Lin et al. (2005) identified several blobs in the outflow and speeds of five of them could be measured, and uncertainties in estimating the local Alfvén speed near the CS could be more or less avoided.

Later, Narukage and Shibata (2006) reported observations of reconnection inflows near the reconnection site after analyzing the EIT data for a set of events. They surveyed the events observed by EIT from 1996 to 2000 and recognized six events that apparently manifested reconnection inflow. The approach used by Yokoyama et al. (2001) to deduce the speed of the reconnection inflow was improved, and the inflow speed measured through the new method was larger than previous ones by a factor of 2.5. Correspondingly, Narukage and Shibata (2006) found that the rate of magnetic reconnection for the events studied, including that investigated by Yokoyama et al. (2001), varied from 0.001 to 0.07 . This result is consistent with those obtained by other authors (e.g., see Table 4 of Narukage and Shibata 2006).

In a work focusing on the reconnecting current sheet above the solar flare, Liu et al. (2010) studied the eruption of a trans-equatorial arcade on 2004 July 29, which resulted in a halo CME and a C2.1 flare. A bright elongated feature extending from the tip of the cusp structure to the CME was observed in the impulsive phase, and was identified with the reconnecting current sheet as predicted by Lin and Forbes (2000). The sheet was seen in the high temperature spectral line Fe XXIV whose emission peaks at $2 \times 10^{7} \mathrm{~K}$. Estimates of the plasma density ranged from $10^{6}$ to $10^{9} \mathrm{~cm}^{-3}$ between the upper and the lower tip of the sheet. These values are consistent with those found by Ko et al. (2003). Furthermore, a merging motion of the disrupting arcade legs was also noticed, and the corresponding inflow speed was about $6 \mathrm{~km} \mathrm{~s}^{-1}$ near the sheet, which is consistent with that obtained by Lin et al. (2005).

Similar to the event studied by Lin et al. (2005), the reconnection inflow was observed in the form of sweeping of the magnetized plasma into the CS region at the beginning of an event occurring on 2010 November 3 started with an erupting flux rope (Savage et al. $2012 \mathrm{~b}$ ). The reconnection outflow was soon observed no more than a minute after the inflow was detected (Fig. 8a). This event was observed by both SDO/AIA in several passbands and RHESSI simultaneously (see also Reeves and Golub 2011; Cheng et al. 2011; 
Bain et al. 2012; Glesener et al. 2013), and a bright and elongated linear feature, as marked in Fig. 5c of Savage et al. (2012b), that was referred to as the candidate CS was recognized within the first few minutes of the eruption. The RHESSI data indicated that the non-thermal component of X-ray emission ( $\geq 25 \mathrm{keV}$ ) peaked first and the thermal-dominated total emission $(\leq 25 \mathrm{keV})$ reached maximum 3 minutes after. The reconnection inflow was first seen in high temperature line AIA $131 \AA$ at speeds ranging from 660 to $690 \mathrm{~km} \mathrm{~s}^{-1}$, and that seen in a low temperature line, say $171 \AA$, did not appear until $4 \sim 7 \mathrm{~min}$ at apparently lower speed $\left(\sim 150-260 \mathrm{~km} \mathrm{~s}^{-1}\right)$. The local Alfvén speed near the CS is most likely in the range from 500 to $3000 \mathrm{~km} \mathrm{~s}^{-1}$, which allows for deviations due to magnetic field and density variations. Using this information, they found that, on average, $M_{A}$ decreased from 0.4 at the beginning to 0.1 about 14 min later, and that the reconnection process is more of the Petschek-type than Sweet-Parker, which indicated the occurrence of fast reconnection.

Features of the reconnection outflow inside the CS were also detected almost at the same time as the inflow was detected in the event. Unlike features of the inflow, however, only those observed in $131 \AA$ and $94 \AA$ channels were more likely to be the outflow along the CS (Fig. 8b), those seen in the 211 and $193 \AA$ bandpasses were not in the typical outflow (SAD or SAD loop [SADL]) morphology, but appeared more like density enhancements traveling down along the legs of newly formed loops. When overlaid on the $131 \AA$ image sequence, the flows in 211 and $193 \AA$ could be seen indeed to move along the loop legs. Because of less clarity, the information about the outflow seen in $94 \AA$ was not included in Fig. 8b. In total 12 outflows were recognized in each of the three AIA wavelengths, $131 \AA$, $211 \AA$, and $193 \AA$. They estimated outflow speeds averaged over 12 outflows (11 sunward and 1 antisunward; the anti-sunward speed $\sim 150 \mathrm{~km} \mathrm{~s}^{-1}$ ) on the plane-of-sky for the SADs/SADLs tracked in $131 \AA$ of $\sim 240 \mathrm{~km} \mathrm{~s}^{-1}$ versus a median value of the speed of $\sim 10^{3} \mathrm{~km} \mathrm{~s}^{-1}$ for the 12211 and $193 \AA$ outflows. It is interesting to note that the retracting structures like SADs and SADLs moved apparently slower than the reconnected plasma flows in the CS.

Recently, both the reconnection inflow and outflow were also reported in a compact flare. Su et al. (2013) studied a flare on 17 August 2011 that was created by collision of two coronal loop systems and observed in both EUV and X-ray emissions. The multi-wavelength EUV observations from SDO/AIA showed inflowing cool loops and newly formed, outflowing hot loops. RHESSI X-ray spectra and images simultaneously displayed the appearance of plasma heated to $>10^{7} \mathrm{~K}$ at the expected locations. The velocity of two loop systems approaching to one another varied from 10 to $70 \mathrm{~km} \mathrm{~s}^{-1}$. In this process, magnetic reconnection between two systems yielded the ejection of the newly formed loops at speed between 90 and $440 \mathrm{~km} \mathrm{~s}^{-1}$. Ignoring the projection effect and assuming the outflow speed to be identified with the local Alfvén speed, Su et al. (2013) eventually ended up with that $M_{A}$ is between 0.05 and 0.5 . These results are similar to those obtained by Takasao et al. (2012) who noticed that the inflow speed was between 12 and $90 \mathrm{~km} \mathrm{~s}^{-1}$, the outflow speed between 220 and $460 \mathrm{~km} \mathrm{~s}^{-1}$, and $M_{A}$ between 0.055 and 0.2 accordingly.

\subsection{Downward Reconnection Outflows}

Observations of plasma flowing out of the reconnection towards the Sun have also been made, and we discuss these results here. McKenzie and Hudson (1999) studied a longlived event that created a group of growing flare loops and a CME. This event was earlier observed by Yohkoh on 20 January 1999. For the first time, they recognized the apparent motions above the post-flare loops, and identified the motion with the reconnection outflow in the CS. Similar mass motions were later observed as well in the 2002 April 21 event by TRACE (e.g., see Innes et al. 2003a, 2003b; Sheeley et al. 2004; Reeves et al. 2008a; and 
detailed discussions below). Comparing the magnetic configurations around and/or related to these mass motions with that in Fig. 2, we believe that McKenzie and Hudson (1999) had observed a CS above the flare loops roughly face-on although observing a CS face-on in the Yohkoh era was not a trivial job. On the other hand, nowadays, with the observations from the SDO/AIA, currently the highest resolution solar observatory capable of coronal measurements, identifying a CS face-on has become less difficult (e.g., see Warren et al. 2011; Savage et al. 2012a, 2012b; McKenzie 2013; Hanneman and Reeves 2014; Doschek et al. 2014).

In the event observed by McKenzie and Hudson (1999), the CS manifested as a fan of spikelike "rays" above the loops. Morphological features of the similar type had been seen in many events by Yohkoh (Švestka et al. 1998), but in this case there was a much clearer view of mass motions in the region above the arcade. McKenzie and Hudson (1999) noticed that the motion indicated the field line retraction but did not see cusp structure on the top of flare loops. This is probably because they observed the CS face-on and the cusp feature could be seen only when the sheet is observed roughly edge-on (see also detailed discussions on this issue by Forbes and Acton 1996). In the late phase of the event, McKenzie and Hudson (1999) found that the downward motion was represented by a dark void in soft X-ray (SXR) moving at speed ranging from 100 to $200 \mathrm{~km} \mathrm{~s}^{-1}$, and that the temperature in the void reached up to $9 \times 10^{6} \mathrm{~K}$, which was slightly hotter than the surrounding fan-shaped supraarcade plasma, and the density was about $10^{8} \mathrm{~cm}^{-3}$. Later, McKenzie (2000) reported 11 long-life CME-associated events observed by Yohkoh/SXT that displayed similar sunward mass motions at speed ranging from 50 to $500 \mathrm{~km} \mathrm{~s}^{-1}$, which were also observed recently by the SDO/AIA (Savage et al. 2012a). Because of the weak signal of the X-ray data in the void, the temperature deduced for the dark void by McKenzie and Hudson (1999) was somewhat suspect.

In the work of Reeves et al. (2008a), these dark voids were also named as SADs. Now terms "SADs", "dark voids" and "dark lanes" are usually used in parallel. Spectroscopic observations indicated for the first time SADs to be voids (Innes et al. 2003a), and by analyzing the data from SDO/AIA with high signal level and increased temporal cadence, Savage et al. (2012a) argued that SADs were actually density depletions left in the wake of thin flux tubes retracting from a reconnection site in the supra-arcade region, and should no longer be considered cross-sections of newly reconnected, large evacuated flux tubes. Hanneman and Reeves (2014) further found that SADs usually had temperatures very similar to or lower than that of the surrounding flare arcades, which ranged from $3.1 \times 10^{6}$ to $1.3 \times 10^{7} \mathrm{~K}$, and that their emission measure was typically about an order of magnitude lower than the nearby arcade. Studies by McKenzie (2013) also displayed evidence of vorticities that varied in time, stagnation points (S-points), and numerous velocity shears in the flows observed in the hot $\left(\sim 10^{7} \mathrm{~K}\right)$ plasma above the flare arcades. By comparing observations with numerical experiments, Innes et al. (2014) suggested that SADs resulted from secondary instabilities of the Rayleigh-Taylor type at the head of reconnection jets.

We need to note here that S. Savage (2014, private communications) keeps pointing out that SADs and plasmoids usually appearing in numerical experiments (e.g., see Bhattacharjee et al. 2009; Huang and Bhattacharjee 2010; Bárta et al. 2011a; Shen et al. 2011; Mei et al. 2012; and references therein) are not the same thing. SADs are low-density regions, while plasmoids show up in simulations as high density structures, and plasmoids appear when we view CSs edge-on, while SADs appear when we view CSs face-on. There are a few papers that discuss theories behind SADs (Cécere et al. 2012; Cassak et al. 2013; Scott et al. 2013; Guo et al. 2014), though these are still not fully accepted.

Following McKenzie and Hudson (1999) and McKenzie (2000), Sheeley and Wang (2002) identified downward motions of the coronal mass at the heliocentrical distance from 
Fig. 9 Height-time maps of the plasma blobs produced in the event of 21 April 2002. The top panel was obtained for the path of the "tadpole" (indicated by arrow in Fig. 1 of Sheeley et al. 2004) and shows steep tracks rapidly decelerating to form new layers of the slowly descending cloud. The bottom panel shows a neighboring region without "tadpoles" and emphasizes the relation between the descending tracks of the plasma cloud and the rising ramp of post-flare loops. From Sheeley et al. (2004)

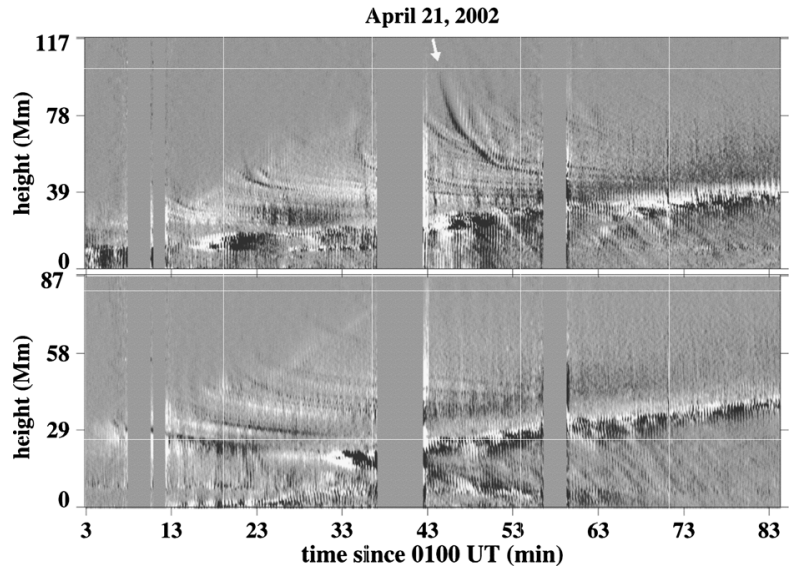

2 to $6 \mathrm{R}_{\odot}$, with each flow at speed varying from 50 to $100 \mathrm{~km} \mathrm{~s}^{-1}$. The event occurring above the west limb on April 21, 2002 provided us a good opportunity to study the sunward reconnection outflows (see Fig. 5 of Reeves et al. 2008a, which displayed an excellent example of the sunward outflow seen in the $195 \AA$ channel with TRACE. The high-temperature response of TRACE at this wavelength provided important information of the hot plasma above the flare loops). These reconnection outflows in the CS were observed in the early phase of the event, so both the temperature and the speed of the plasma flow were high and reached up to $10^{3} \mathrm{~km} \mathrm{~s}^{-1}$ and $10^{7} \mathrm{~K}$, respectively.

In addition to the flow itself, the deceleration of flow was also observed as a result of interaction with the closed flare loops below. Figure 9 displays the time profiles of the void location inside the current sheet, which shows the void motion and its rapid deceleration (see the dark void specified by the white arrow). This void moved at speed of about $600 \mathrm{~km} \mathrm{~s}^{-1}$, and then underwent a significant deceleration at up to $1.5 \times 10^{3} \mathrm{~m} \mathrm{~s}^{-2}$, which suggested a violent interaction between the reconnection outflow and the closed flare loops, and might further imply the formation of the termination shock on the top of the loop system (Aurass and Mann 2004; Aurass et al. 2006, 2011). So far, on the other hand, whether a termination shock could form as a result of interaction of the reconnection outflow with the flare loop is still an open question, and more detailed investigations are surely needed (e.g., see also discussions of Gao et al. 2014). We note further here that the deceleration of an outflow and the formation of a termination shock might also occur without the loop top obstacle, the increase in the plasma density in the related region may play the role of the obstacle.

Asai et al. (2004) presented a detailed examination of downward motions of the plasma above flare loops observed in the 2002 July 23 event. This event produced an X-4.8 flare and a fast CME at speed of $2.6 \times 10^{3} \mathrm{~km} \mathrm{~s}^{-1}$, which were observed by several instruments both in space and on the ground, including TRACE, RHESSI, NoRH, UVCS and LASCO. The EUV images obtained by TRACE showed dark downward flows (sunward motions) above the postflare loops, not only in the decay phase but also in the impulsive and main phases. They also found that the times when the downward flows started to be seen corresponded to the times when bursts of nonthermal emission in HXRs and microwaves were produced. This implies that the downward motions of the plasma occurred when magnetic energy was quickly released as a result of magnetic reconnection, and it is worth noting that the downflows in this flare was observed in the impulsive phase. To our knowledge, the downflows occurring in the impulsive phase are not often reported. 
Similar downward flows over the top of flare loops were also observed in both HXR (25 50 keV and 12 25 keV) and SXR (6 12 keV) by RHESSI in 3 events studied by Sui and Holman (2003) and Sui et al. (2004). In these events, high temperature loops or the loops observed in high energy bands $(25 \sim 50 \mathrm{keV}$ or $12 \sim 25 \mathrm{keV})$ were seen above the low temperature loops or the loops observed in low energy bands $(6 \sim 12 \mathrm{keV})$. Meanwhile, the loop system observed in both bands manifested downward motion in the HXR impulsive phase at speed ranging from 8 to $32 \mathrm{~km} \mathrm{~s}^{-1}$ with the loops seen in high energy band moving faster than those in the low energy band. The flare loops observed in the radio bands with frequencies ranging from 17 to $32 \mathrm{GHz}$ as well as those observed in $193 \AA$ by TRACE confirmed such a downward motion of the flare loop system in the early stage of the eruption (Li and Gan 2005, 2006). Veronig et al. (2006) saw the same feature when studying an X-3.9 flare such that the mean downward velocities range from $14 \mathrm{~km} \mathrm{~s}^{-1}$ in the RHESSI $10-15 \mathrm{keV}$ energy band to $45 \mathrm{~km} \mathrm{~s}^{-1}$ in the $25-30 \mathrm{keV}$ band, and that observed by the SXI instrument was $12 \mathrm{~km} \mathrm{~s}^{-1}$. Reeves et al. (2008b) studied a B-class and a C-class flare, and found that individual loops moved downward at speeds ranging from 5 to $48 \mathrm{~km} \mathrm{~s}^{-1}$ and from 2 to $4 \mathrm{~km} \mathrm{~s}^{-1}$, respectively. Their estimates showed that measurements of the shrinkage in these two events were between $17 \%$ and $27 \%$. Recent work by Liu et al. (2013) on an M7.7 flare showed that the speed of such a descent motion of the overall X-ray and EUV loop-top varied from 7 to $23 \mathrm{~km} \mathrm{~s}^{-1}$.

The downward motion observed in the above events could be a combination of the plasma flow and the shrinkage of flare loop system created by magnetic reconnection in the CS (e.g., see also Švestka et al. 1987; Lin et al. 1995; Forbes and Acton 1996; Lin 2004; Reeves et al. 2008a, 2008b; Liu 2013; Liu et al. 2013). Both of them are the products of magnetic reconnection, but the former is the motion of plasma and the latter is the motion of magnetic structures. Observationally, they mixed together and could hardly be distinguished from one another previously due to the low spatial resolution and time cadence of the instruments. SDO/AIA allows for unprecedented scrutiny of the SADs. Savage et al. (2012a) showed that SADs, which once were thought to be identifiable as the shrinking loops themselves, appeared to be "side effects" of the passage of the loops through the supra-arcade plasma, and demonstrated the observational difference of SADs and loop shrinkage.

The shrinkage of the loop system was observed by Sui et al. (2004) for the first time in HXR. It usually occurs within a short period at the beginning of the impulsive phase, and then the loop system displays apparent upward motion as usually seen in two-ribbon flares. Sui et al. (2004) suggested that this motion is due to the fact that the current sheet develops as a result of the collapse of the X-type neutral point. This scenario was first introduced by Dungey (1953) such that the X-type neutral point is locally unstable if the source region of the relevant configuration could move freely, and the X-point would consequently collapse and develop to a current sheet with a Y-type neutral point (or a reversed current and singularity) at each end (see also Figs. 2.1 and 2.2 of Priest and Forbes 2000). In this scenario, the formation of the reversed current may push the flare loop system to move downward at the very beginning of the event.

Considering the fact that the magnetic configuration is severely stretched in the eruptive process, we believe that the current sheet should be produced as the local magnetic structure of the X-point is stretched (see Fig. 2.10b of Priest and Forbes 2000, which demonstrated the bifurcation of an X-point in response to motions of sources that stretch the magnetic structure around the X-point) instead of collapses (see Fig. 2.10c of Priest and Forbes 2000 for the bifurcation of the X-point due to squeezing of the related structure). Analytic studies (Forbes and Isenberg 1991; Isenberg et al. 1993; Forbes and Priest 1995; Lin and Forbes 2000) indicated that the catastrophic loss of equilibrium in the coronal magnetic configuration that includes a current-carrying flux rope thrusts the flux rope outward, 
severely stretching the magnetic structure around the flux rope and resulting in the formation of an X-point and then a CS with a Y-point at each end. Numerical experiments of Mei et al. (2012) demonstrate clearly this process occurring in a disrupting magnetic configuration. Because stretching directly produces a low pressure region near the CS, and both magnetic field and plasma around the CS between the two Y-points are pushed toward the CS, forming the reconnection inflow all along the CS, by the pressure gradient. Since the pressure gradient is not just confined to the region near the Y-point, but exists all along the CS, the reconnection inflow is expected all along the CS. Because the CS is created by stretching, a CS with reversed current and singularity may not be produced, and the downward motion of the flare loop system at the beginning of the impulsive phase should be due to the other reasons. Lin (2004) ascribed this motion to the standard shrinkage of the newly formed closed flare loops.

In a two-ribbon flare process, individual flare loops do not stay at the height when they are newly formed, but decrease to a lower altitude at which their shape is more potential, and the earlier the loops are formed, the faster the loops shrink (Lin et al. 1995; Lin 2004). In the impulsive phase of HXR, the shrinkage of individual loops is much more significant than the growth of the loop system resulted from accumulations of individual loops such that the speed of the former could be close to $200 \mathrm{~km} \mathrm{~s}^{-1}$, and that of the latter is less than $100 \mathrm{~km} \mathrm{~s}^{-1}$. In reality, manifestations of the two motions are mixed with one another and the highly dynamic behavior of loops in the early stage of the flare makes distinguishing them from one another very difficult, so the component that is moving faster dominates the observational consequence. But this question is still open and more investigations in detail are necessary.

After having studied all the flare events collected, Savage and McKenzie (2011) identified 62 cases of sunward plasma flows above the flare loops. These events had been observed by Yohkoh, TRACE, SOHO/LASCO, SOHO/SUMER, and Hinode/XRT over almost 20 years. They looked into 35 events among which 10 displayed the mixture of the sunward flow and the loop shrinkage, and 25 showed the pure plasma flow in the current sheet. In individual events, the largest number of pure plasmoid motions identified in the sheet was 25 , and the largest number of the mixture of plasmoid motions and shrinkages was 60 . Overall, the average velocity of these motions was about $150 \mathrm{~km} \mathrm{~s}^{-1}$. Furthermore, Hara et al. (2011) found that the speed of the reconnection outflow near the top of the flare loop system was around $200 \mathrm{~km} \mathrm{~s}^{-1}$ by analyzing the data from Hinode/EIS, and Warren et al. (2011) obtained a speed close to $150 \mathrm{~km} \mathrm{~s}^{-1}$ from SDO/AIA data. Compared with the anti-sunward flow, the sunward flow is apparently slow. Shen et al. (2011) pointed out that this is generally true because the sunward flow is always slowed down by its interaction with the closed flare loops below. Studying the SDO/AIA data, Takasao et al. (2012) brought the speed of the anti-sunward flow to the range from 220 to $460 \mathrm{~km} \mathrm{~s}^{-1}$, and that of the sunward flow to the range from 250 to $280 \mathrm{~km} \mathrm{~s}^{-1}$.

\subsection{Upward Reconnection Outflows}

In this part of work, we discuss the upward (anti-sunward) outflows in the CS. After Ciaravella et al. (2002) confirmed observationally for the first time the existence of the reconnection region behind the CME where a CS should be located as predicted by Lin and Forbes (2000), Ko et al. (2003) performed the first quantitative study of the reconnection outflow leaving the Sun in the 2002 January 8 event although they were not the first to observe the anti-sunward reconnection outflow (e.g., see discussions by Webb et al. 2003; Simnett 2004 for details). The event studied by Ko et al. (2003) occurred on the east limb 
Fig. 10 Heights of five well-recognized blobs in the 2002 January 8 event versus time. The corresponding distances of two successive blobs are also indicated. From Lin et al. (2007)

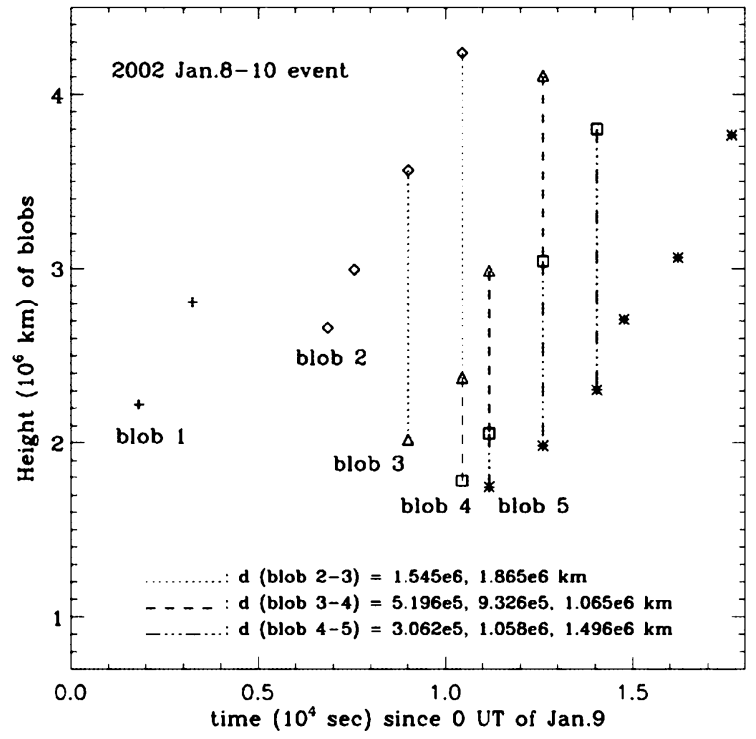

and produced a fast CME associated with a growing SXR flare loop system (the main part of the flare was behind the limb so it is hard to determine the class of the flare). The advantage of studying this event is that it occurred on the limb and the current sheet above the flare loop system could be observed edge on, so that enough emission measure from the sheet would be collected and reliable diagnostics of plasma properties of the sheet could be performed.

Combining the data from the UVCS, LASCO, EIT, and CDS on board SOHO, as well as those from the MLSO MK4 coronameter (Elmore et al. 2003), Ko et al. (2003) investigated the morphological and dynamical properties of this event, and the physical properties of the current sheet. The velocity and acceleration of the CME reached up to $1.8 \times 10^{3} \mathrm{~km} \mathrm{~s}^{-1}$ and $1 \mathrm{~km} \mathrm{~s}^{-2}$, respectively. The acceleration is found to occur mainly at the lower corona $\left(<2.76 \mathrm{R}_{\odot}\right)$. The post-CME loop systems showed behaviors of both postflare loops (upward motion with decreasing speed) and soft X-ray giant arches (upward motion with constant speed, or acceleration) according to the definition of Švestka et al. (1987). In the current sheet, the presence of highly ionized ions, such as [Fe XVIII] and [Ca XIV], suggested temperature as high as (3-6) $\times 10^{6} \mathrm{~K}$, and the plasma outflows had speeds ranging from 300 to $650 \mathrm{~km} \mathrm{~s}^{-1}$. Absolute elemental abundances in the CS showed a strong first ionization potential effect similar to that found in the active region streamers. The magnetic field strength in the vicinity of the CS was found to be of the order of $1 \mathrm{G}$.

In the images (movies) of LASCO, the anti-sunward plasma flows could be recognized by tracking a group of plasma blobs (or plasmoids) moving along the current sheet. Figure 18 of Ko et al. (2003) displays two examples, one showed accelerating motion pattern, and another one showed tendency of slowing down. Selecting 5 blobs with sharp edge that could be easily recognized and following their motions, Lin et al. (2007) plotted their heights at different times as shown in Fig. 10, and further deduced the velocity of each blob, which is between 300 and $650 \mathrm{~km} \mathrm{~s}^{-1}$. The distance of every two adjacent blobs is also marked in Fig. 10, and the importance of these distances will be discussed later.

In the event of 18 November 2003, many blobs were also recognized in the LASCO data (see Fig. 11), and the speeds of the plasma blobs moving away from the Sun along the current sheet were between 460 and $1075 \mathrm{~km} \mathrm{~s}^{-1}$ [see also Fig. 8 of Lin et al. 2005 that 
Fig. 11 LASCO C2 and C3 images. (a) A helmet streamer exists in the region that the CME will go through. With rapid expansion of the CME, (b) the helmet streamer is severely deflected from outside, and (c, d) the southern leg of the CME (disrupting arcade) merges with the helmet streamer, forming a new helmet streamer. A couple of blobs of the reconnected plasma flowing along the current sheet can also be recognized in (c) and (d). All times are UT. From Lin et al. (2007)
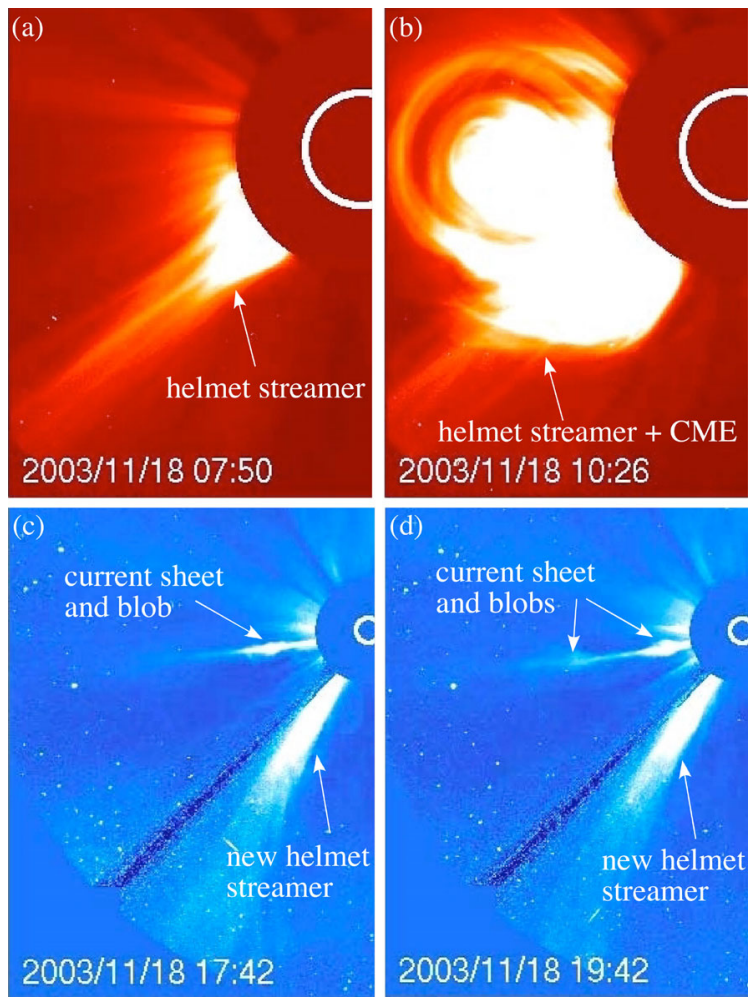

is a composite of LASCO C2 (at 17:50 UT), MK4 (17:49 UT), and EIT $195 \AA$ (17:48 UT) images of the event, clearly illustrating the positions of the post-flare/CME loops, cusp point, and extended current sheet, which resembles the typical Kopp-Pneuman configuration for major flares]. Figure 11 indicates that the CS could extend up to $20 \mathrm{R}_{\odot}$. According to Forbes and Lin (2000), Lin and Forbes (2000), and Lin (2002), the upper (outer) tip of the CS is right behind the CME rear edge, and the distance between these two locations depends on both the rate of magnetic reconnection inside the CS as well as the local Alfvén speed near the CS. Because of the observational limitations, the CS became too diffuse to be recognized far away from the Sun. Therefore, it is hard to tell where the CS terminates at the far end although it is now well known the lower tip or the near end of the CS terminates at the top of the associated flare loop system. Figure 11 shows that the far end of the CS disappeared at around $20 \mathrm{R}_{\odot}$, but it does not necessarily mean that it terminated there. More advanced techniques and higher sensitivity detectors are needed in order to resolve this problem in the future.

After having analyzed the LASCO data obtained in the time interval between 1996 and 2009, Song et al. (2012) found more plasma blobs flowing inside the post-CME CS. The speeds of these blobs spread over the range from 200 to $10^{3} \mathrm{~km} \mathrm{~s}^{-1}$. In recent works by Liu (2013) and by Liu et al. (2013) on an event that produced an M7.7 flare and a fast CME at speed $>10^{3} \mathrm{~km} \mathrm{~s}^{-1}$ (see also Patsourakos et al. 2013), the reconnection outflow was reported to leave the Sun along the current sheet over the flare loop system at speed up to $1.4 \times 10^{3} \mathrm{~km} \mathrm{~s}^{-1}$. These results are consistent with those of Ko et al. (2003) and Lin et al. (2005), and suggest a positive correlation of the outflow speed with the class of the associated flare. In addition, Song et al. (2012) also studied the plasma blobs flowing inside the 
helmet streamer, and noticed that these blobs are apparently slower than those inside the CME current sheet. They concluded that the difference in velocities of plasma blobs could be ascribed to the reconnection processes occurring in the different environments. Similar phenomenon was also observed in numerical experiments on the reconnection process taking place in two-ribbon flares (see also Forbes and Malherbe 1991; Riley et al. 2007; Shen et al. 2011).

We need to note here that the CS inside the helmet streamer is very different from the post-CME CS in their dynamic property although they are the same in the intrinsic physical property. The former is nearly stationary and could exist for a long time, but the latter is highly dynamic and is produced in the eruptive process. We note here that "stationary" means that the helmet streamer and the CS inside already existed before the CME and continued to exist after the CME [see the structure located at the polar angle (PA) of around $140^{\circ}$ in all the panels of Fig. 11], and that "dynamic" means that the post-CME CS did not exist beforehand, but was created and developed during the eruption (see the structure located around $\mathrm{PA}=100^{\circ}$ in Figs. $11 \mathrm{c}$ and $11 \mathrm{~d}$, which did not appear in Figs. 11a and 11b). Panels in Fig. 12 display LASCO C2 images of the same process that had been enhanced by the wavelet technique, which further indicates that discriminating a post-CME CS from a streamer CS is not difficult, although in both cases the tearing mode instability could take place in the same fashion (e.g., see also Einaudi et al. 2001) since the occurrence of the tearing mode depends on the length to thickness ratio of the CS (e.g., see Furth et al. 1963; Loureiro et al. 2007; Ni et al. 2010; Shen et al. 2011; Bárta et al. 2011a, 2011b; Mei et al. 2012) no matter whether the CS is stretched by the expanding solar wind, or by the disrupting coronal magnetic field.

In addition, observations in the radio band revealed dynamical features of plasma blobs inside the CS. When studying the trans-equatorial coronal loop eruption occurring on 29 July 2004, Liu et al. (2010) found that a current sheet was produced at the early stage of the event, and that radio observations made in space and on the ground revealed important information on the CS and dynamic features of plasma inside the sheet. First of all, a set of DPSs at metric frequencies was detected (see Fig. 2 of Liu et al. 2010). Compared with those usually observed at the decimetric frequency (Kliem et al. 2000), the DPS observed in this event was obviously produced at higher altitudes and displayed the pattern of the anti-sunward motion in group, which was suggestive of tearing of the sheet and the resultant magnetic islands moving away from the Sun along the sheet. The fine details included in DPSs indicated interactions of energetic electrons trapped in the magnetic islands (or plasma blobs). In the associated LASCO data, the motion of plasma blobs was also recognized at speed of $\sim 360 \mathrm{~km} \mathrm{~s}^{-1}$. Second, a type III radio burst was detected at frequencies ranging from 1.08 to $13.83 \mathrm{MHz}$, which implied the escape of some energetic electrons from trapping to the high corona and interplanetary space.

\subsection{Bi-Directional Reconnection Outflows Observed in a Single Event}

Conservation of the momentum of the plasma flow determines that the bi-directional outflow is the ubiquitous consequence of the magnetic reconnection process no matter in what type of reconnection. As we clarified earlier, the term "current sheet" used in this and our previous works possesses a general meaning such that it refers to the region where fast dissipation/reconnection of the magnetic field takes place. In most cases, we are able to observe the reconnection outflow in one direction, and it is usually difficult to identify bi-directional flows simultaneously in a single event. This is because the bifurcation point of the reconnection outflow usually appears at low altitude, say lower than $1 \mathrm{R}_{\odot}$ as suggested by Shen et al. 

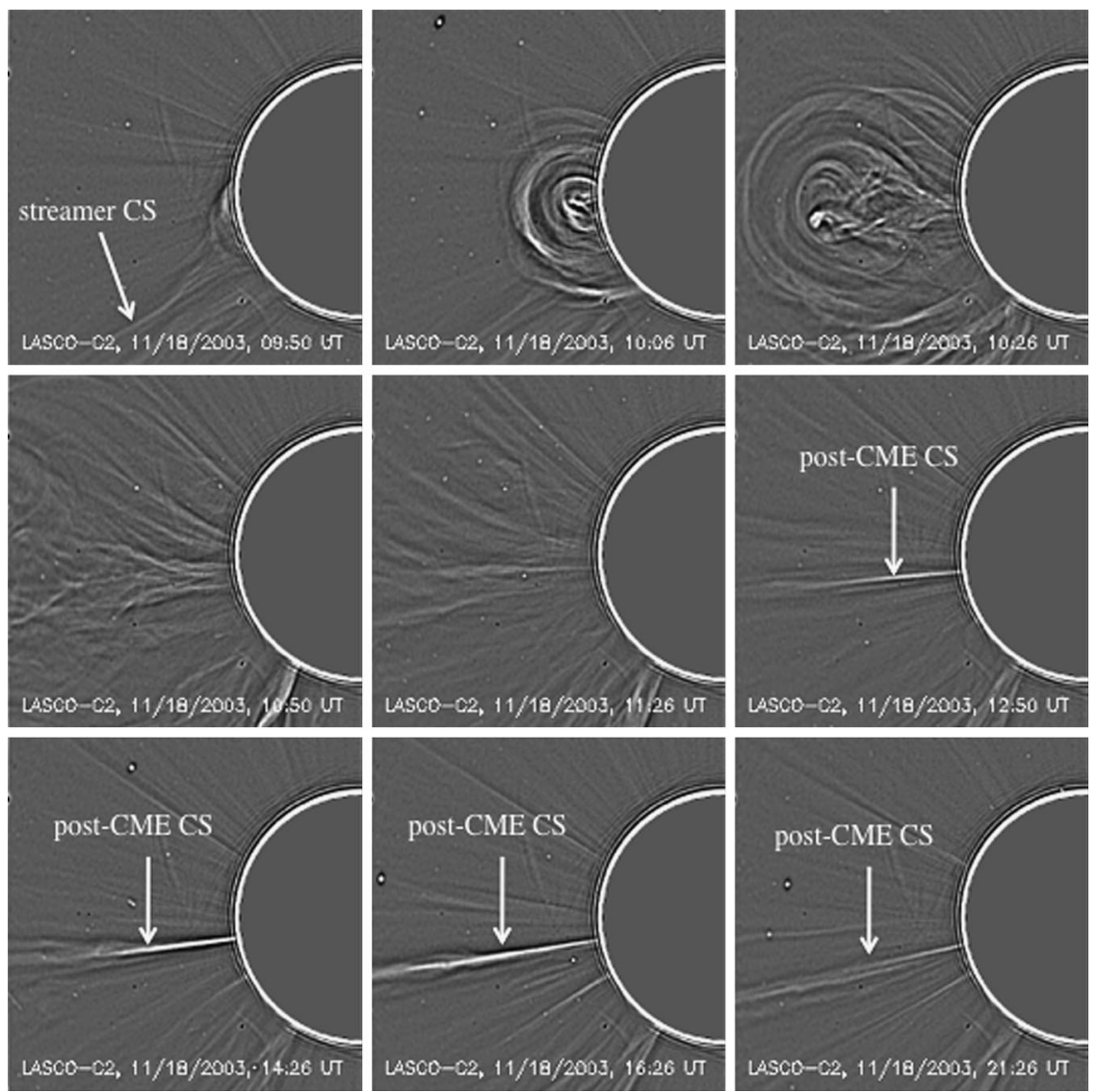

Fig. 12 LASCO C2 images of the 2003 November 18 event that have been enhanced by the wavelet technique, which is good at emphasizing the fine features of large-scale structures. The leading edge, cavity, and core of the CME are easily recognizable. The post-CME and streamer CSs have been apparently specified. From Lin et al. (2005)

(2011). So the sunward flow in the eruptive event usually cannot be observed by a coronagraph with a large occulting disk like LASCO/C2 and C3, while instruments with FOV near the solar surface can only recognize the anti-sunward motions. In the streamer detachment event, on the other hand, the bi-directional reconnection outflows have indeed been reported with LASCO data and defined as "in/out pairs" by Sheeley and Wang (2007). This indicates that, unlike that in the eruptive event, the bifurcation point of the reconnection outflows in the streamer detachment event is located high in the corona.

To our knowledge, the eruptive event on April 9, 2008 is the first one in which both sunward and anti-sunward reconnection outflows were observed simultaneously (e.g., see Savage et al. 2010). This event took place over the active region AR 10989, and produced an $\mathrm{X}$-class flare and a CME. The active region was located about $23^{\circ}$ behind the west limb when the event occurred, and several instruments in space observed both the flare and the associated CME. These instruments include XRT on Hinode, TRACE, SECCHI on STEREO A, and LASCO on SOHO. GOES did not classify the flare because most of the flare was located behind the limb. Occulting of the brightest structures of the flare by the solar limb allows the 
Fig. 13 The CS in the region between two arrows developed in the "cart-wheel" event on April 9, 2008. The figure in the left panel was taken at 11:08:42 UT, and that in the right panel was taken at 17:31:55 UT. The change in the orientation of the current sheet resulted from the self-adjustment of the associated magnetic configuration in the eruption at speed of around $4^{\circ} \mathrm{h}^{-1}$. From Savage et al. (2010)

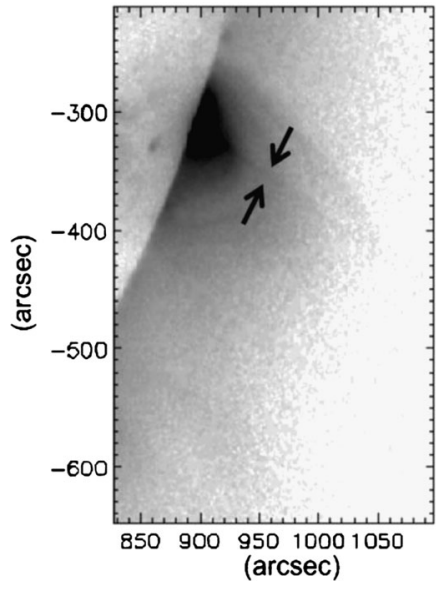

less bright structures above the active region to be recognized more easily. As the eruption was in progress, the CME moved away from the Sun like a cart wheel, so this CME is also known as the "cart-wheel CME".

Focusing on the data from XRT and LASCO, and using the data from SECCHI for reference, Savage et al. (2010) found a large amount of mass seen in EUV and SXR to be expelled from the Sun at speed ranging from 80 to $180 \mathrm{~km} \mathrm{~s}^{-1}$ in the time interval from 09:16 and 10:11 UT. Structures of the associated flare that could be seen were about $7 \times 10^{4} \mathrm{~km}$ above the limb. The resultant white-light CME entered the FOV of LASCO at 11:06 UT and swept the FOV of LASCO C2 at speed of $\sim 450 \mathrm{~km} \mathrm{~s}^{-1}$. Landi et al. (2010) studied the initial acceleration phase of the CME in the same event, analyzed the physical properties of the CME core, and their data set included all other manifestations associated a CME: flare loops, CS, and coronal dimming. Landi et al. (2012) extended the work of Landi et al. (2010) to the diagnoses of plasma behind the CME, including that in the CS, to complement the works by Savage et al. (2010) and Patsourakos and Vourlidas (2011) on the same event.

After the CME left the FOV of XRT, a set of the enhanced images (or movies) displayed sunward and anti-sunward plasma flows, together with the shrinkage of flare loops. Savage et al. (2010) noticed that the plasma flows were along a thin bright feature (see Fig. 13), which became more apparent after 11:00 UT and displaced $25^{\circ}$ southward within 6.5 hours at speed of about $4^{\circ} \mathrm{h}^{-1}$. The rotation of the Sun at the corresponding latitude is at speed of $0.6^{\circ} \mathrm{h}^{-1}$, which could not account for the displacement, so the motion of the thin structure should be due to the self-adjustment of the disrupting magnetic configuration as suggested by Ko et al. (2003).

In the XRT FOV, this structure was thin and located between the CME and the flare. Comparing it with those similar ones observed previously (Ciaravella et al. 2002; Ko et al. 2003; Webb et al. 2003; Lin et al. 2005; Bemporad et al. 2006), we are able to identify this structure with the $\mathrm{CME} /$ flare current sheet. What is especially interesting was that a set of plasma blobs flowing in the sheet were seen between 12:18 and 12:35 UT. These blobs appeared to move bi-directionally. The blobs that were selected for further analyses were recognized by naked eyes because of the lower signal-noise ratio of the data. From 11:11 to 17:24 UT, totally 16 plasma blobs were identified with 3 moving sunward and 13 anti-sunward.

Analyzing trajectories and motions of these 16 blobs moving along the current sheet, Savage et al. (2010) found that the velocity of these blobs ranged from 21 to $165 \mathrm{~km} \mathrm{~s}^{-1}$ 
with the mean value of $120 \mathrm{~km} \mathrm{~s}^{-1}$ and an average of $109 \mathrm{~km} \mathrm{~s}^{-1}$ for both sunward and antisunward motions after correcting the projection effects. Overall, the anti-sunward motion was faster than the sunward one, and all the sunward motions were slower than the free-fall speed, which indicated that the motion of the plasma blob was somehow blocked. As we mentioned earlier in this work, slowing down of the downward reconnecting outflow could be due to interactions of the flow with the forward plasma of increasing density, and the turbulence produced by such an interaction might impede the flow as well.

On the other hand, the observed downward motion may as well include a component that comes from the shrinkage of flare loops below the current sheet. Savage et al. (2010) pointed out that the SAD motion at the slowest velocity of $21 \mathrm{~km} \mathrm{~s}^{-1}$ in the event they studied could be of the shrinkage of the flare loops below the current sheet (see also Lin et al. 1995; Lin 2004) rather than the downward reconnection outflows. (This issue seems more complex than expected. In another flare event studied by Savage et al. 2012a, SADs were found to be the "side effects" of the passage of loops through the supra-arcade plasma. More discussions on this issue can be found in Savage et al. 2012b.) Similar events were analyzed by Sui et al. (2005) and Saint-Hilaire et al. (2009), in which the X-ray emission sources in the corona appeared to move both downwards and outwards with time. The outgoing blobs with HXR emission, indicating a very hot source of $\sim 10^{7}-10^{8} \mathrm{~K}$, were identified with the plasma flows, and those moving downward were ascribed to the shrinkage of the newly formed flare loops. Recent work by Liu et al. (2013) indicated that the speed of the loop shrinkage could reach up to $300 \mathrm{~km} \mathrm{~s}^{-1}$, which might be an important issue that is worth extra attention when we study the supra-arcade down flows as a result of magnetic reconnection.

For the blobs moving away from the Sun, tracking their motion becomes difficult after they have left the FOV of XRT, but is still doable by combining the data from LASCO C2 and C3. Savage et al. (2010) performed an extrapolation from the XRT images, estimated roughly where a specific blob might appear in LASCO images, and then located that blob in LASCO images. Therefore, the information from XRT and LASCO could be put together, giving a comprehensive scenario of the blob motion along the current sheet in a long period. Savage et al. (2010) noticed that velocities of blobs entering LASCO FOV from XRT FOV remained nearly unchanged.

Similar plasma flows due to reconnection in the eruption were continuously observed later. Takasao et al. (2012) reported observations and studies of a C4.2 flare occurring on 2010 August 18 on the northwest limb. Flare loops developed in this event could be seen in both hot $\left(>7 \times 10^{6} \mathrm{~K}\right)$ and cool $\left(4 \times 10^{5} \mathrm{~K}\right)$ temperature spectral lines, and a sheet structure could be recognized to extend from the top of flare loops to the outermost corona, and bi-directional plasma flows were identified in both low and high temperatures images, which suggested the wide range of the plasma temperature inside the sheet as observed in previous events (e.g., see McKenzie and Hudson 1999; Innes et al. 2003a; Asai et al. 2004). Speeds of the anti-sunward flow were found between 220 and $480 \mathrm{~km} \mathrm{~s}^{-1}$, those of sunward flow were between 250 and $280 \mathrm{~km} \mathrm{~s}^{-1}$, and significant deceleration of the sunward flow was also detected as the flow approached to flare loops. Interactions of the flow with flare loops eventually slowed the flow to the speed of $70-80 \mathrm{~km} \mathrm{~s}^{-1}$. Observations by different instruments in various wavelengths, for example Yohkoh/SXT (Kolomański and Karlický 2007) and RHESSI (Milligan et al. 2010), as well as numerical experiments revealed the same information (see also Shen et al. 2011; Mei et al. 2012).

In addition to the above observations at optical, EUV, and X-ray wavelengths, together with the associated numerical experiments, it is worthy to note that Kliem et al. (2000) suggested that plasma blobs inside a CS could be "seen" in the radio band via some specific radio characteristics. Theses characteristics include impulsive structures with slowly drifting 
property on the radio dynamic spectrum, which results from energetic particles trapped in the magnetic islands, or plasma blobs, inside CS. Recent studies by Aurass et al. (2011) and Gao et al. (2014) confirmed the idea of Kliem et al. (2000). Karlický et al. (2010) noticed that the coalescence of plasmoids could even account for the occurrence of DPSs observed in solar flares. Gao et al. (2014) investigated the spectral fine structure of a solar radio burst in a CME/flare event on 2004 December 1. The data displayed zebra-like emission patterns with weak polarization, including positive, negative and zero frequency drifting stripes, during the impulsive phase of the event. They noticed that the process of the formation and the motion of plasma blobs inside reconnecting CS was similar to what occurred in the event observed by Aurass et al. (2011).

\subsection{Outflows Observed During Laser-Driven Reconnection}

Before ending our discussions on the reconnection outflow, we turn to the topic of the laserdriven reconnection process taking place in the laboratory to look into detailed features and physical properties of the reconnection region. Since performing in situ measurements for various parameters and the energetic particles in the reconnection region of the solar flare is impossible (at least in the foreseeable future) although performing the similar measurements in the Earth's magnetosphere environment has been done for decades (see Eastwood et al. 2007; Retinó et al. 2007, 2008; Huang et al. 2012; and references therein), conducting alternative laboratory experiments becomes a very viable approach.

Recently, the field of laboratory astrophysics has quickly developed (Remington et al. 2006). In this field, experiments are performed in laboratories, simulating various astrophysical processes, probing important features and key properties of these processes, to allow us to examine the physics of these processes. Although systems in different environments possess very different scales that can vary over a huge range, scaling laws of plasma and MHD processes allow us to relate them to the same process in many cases (Ryutov et al. 2000). Laser-driven magnetic reconnection (LDMR, Nilson et al. 2008; Li et al. 2007; Zhong et al. 2010) is one of the most significant topics of the laboratory astrophysics, booms and draws extensive attention lately.

Referring to solar X-ray observations, some very similar images were also found in a recent LDMR experiment. Faraday rotation method (Stamper and Ripin 1975) measured the magnetic field in laser-produced plasmas, which can be up to a megaGauss. At that time, the structure of magnetic field was not very clear until the proton radiography technique became available. With this technique, the magnetic field is produced in the plasma bubble due to the Biermann battery effect (see Biermann 1950; Roxburgh 1966; Widrow 2002) can be measured/detected. Such a magnetic field resulting from the anisotropic pressure in the plasma when a solid target is intensively irradiated by the long pulse laser and evaporates (nanosecond laser, with intensity of around $10^{15} \mathrm{~W} \mathrm{~cm}^{-2}$ ). Regarding the Biermann battery effect, interested readers are referred to a review by Widrow (2002) for more details.

Yates et al. (1982) realized the occurrence of magnetic reconnection when an unexpected $\mathrm{X}$-ray emission was observed between two laser spots in a multi-beams experiment. As the two bubbles expanded laterally and encountered each other with oppositely directed magnetic fields, reconnection took place and the field lines were topologically rearranged in the diffusion region. Later, with the proton radiography technique, Nilson et al. (2006) and Li et al. (2007) diagnosed the LDMR, and some striking features were found, such as the collimated jets and magnetic null point in the diffusion region.

With long pulse lasers, Zhong et al. (2010) constructed LDMR to model the loop-top $\mathrm{X}$-ray source and outflows, which are often observed in solar flares. In their experiments, 


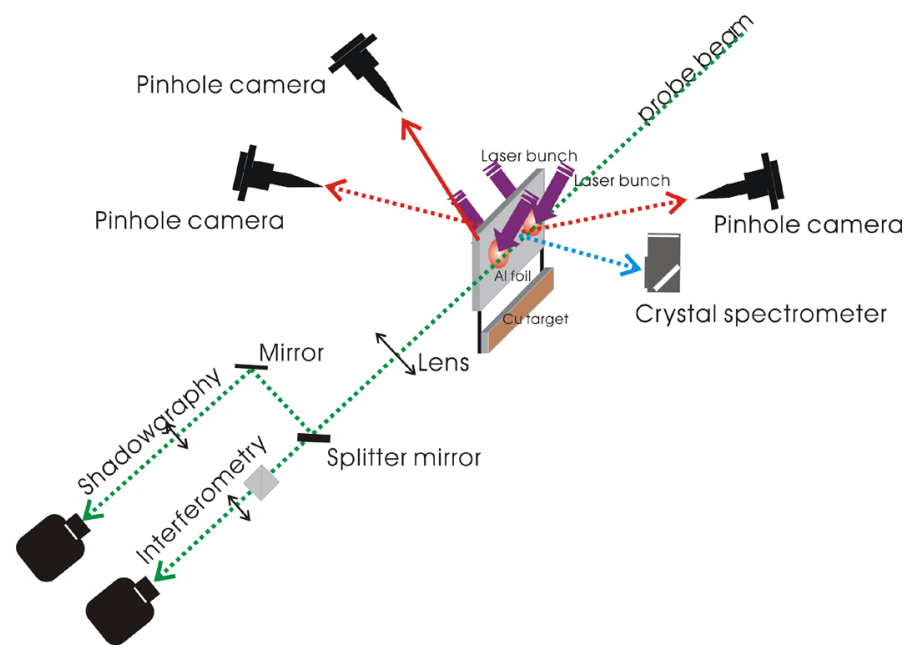

Fig. 14 Experimental set-up for laser-driven magnetic reconnection performed by Zhong et al. (2010). Four bunches of long pulse ( $1 \mathrm{~ns}$ ) lasers are focused on both sides of a thin Al foil target. Shadowgraphy and interferometry are used to diagnose the plasma evolution with a short pulse (120 ps) probe beam (shown as a green dotted line). The MR occurs between the two laser focus spots, and is detected by three X-ray pinhole cameras. The reconnection outflow/jet can thus interact with a pre-set $\mathrm{Cu}$ target. X-ray spectra from the heated plasma are recorded by a crystal spectrometer set in front of the target. From Zhong et al. (2010)

two aluminum $(\mathrm{Al})$ foil targets were placed on the same plane, and a copper $(\mathrm{Cu})$ target was placed right below the Al targets with its plane perpendicular to the Al plane (Fig. 14). Two plasma blobs were created as the Al targets were irradiated by two intense laser beams. Magnetic fields of opposite polarity associated with the plasma blobs moved toward each other as the blobs expanded, and magnetic reconnection took place between them. Two outflows were observed in the experiments. Downward outflow directly collided the $\mathrm{Cu}$ target and produced a very hot X-ray spot, which was used to model the loop-top source of the X-ray emission observed in the two-ribbon flare (Fig. 15). The flow velocity was directly measured to be $400 \mathrm{~km} \mathrm{~s}^{-1}$, which agrees well with the typical Alfvén speed in the lab environment.

However, it is not straightforward to extrapolate directly the phenomena occurring in laboratory to those observed on the Sun because of the huge difference in scales between two environments. By applying the MHD scaling laws, on the other hand, they found that the physical parameters in solar flare and laser plasma systems are very similar, such that the characteristic length scales change from thousands of kilometers to hundreds of micrometers. Therefore, the output of their experiments as shown in Fig. 15 is considered a laboratory counterpart of the solar flare. Another important point drawn from experiments was that the reconnection process could be easily controlled by adjusting the experimental parameters. Namely, they produced inclined outflows with two asymmetrically arranged lasers, which could accordingly produce asymmetrical magnetic fields. Therefore, a "benchtop solar flare" could always be created in the laboratory according to the requirement and duplicate various important processes and/or phenomena that may take place in the solar eruption, and even those occurring in the deep universe.

In addition to helping study the solar flare, the experiment of the laser-driven magnetic reconnection may help us look into details of coalescence of plasmoids inside the reconnecting CS as well because the hot X-ray spot appearing in the experiment of Zhong et al. 

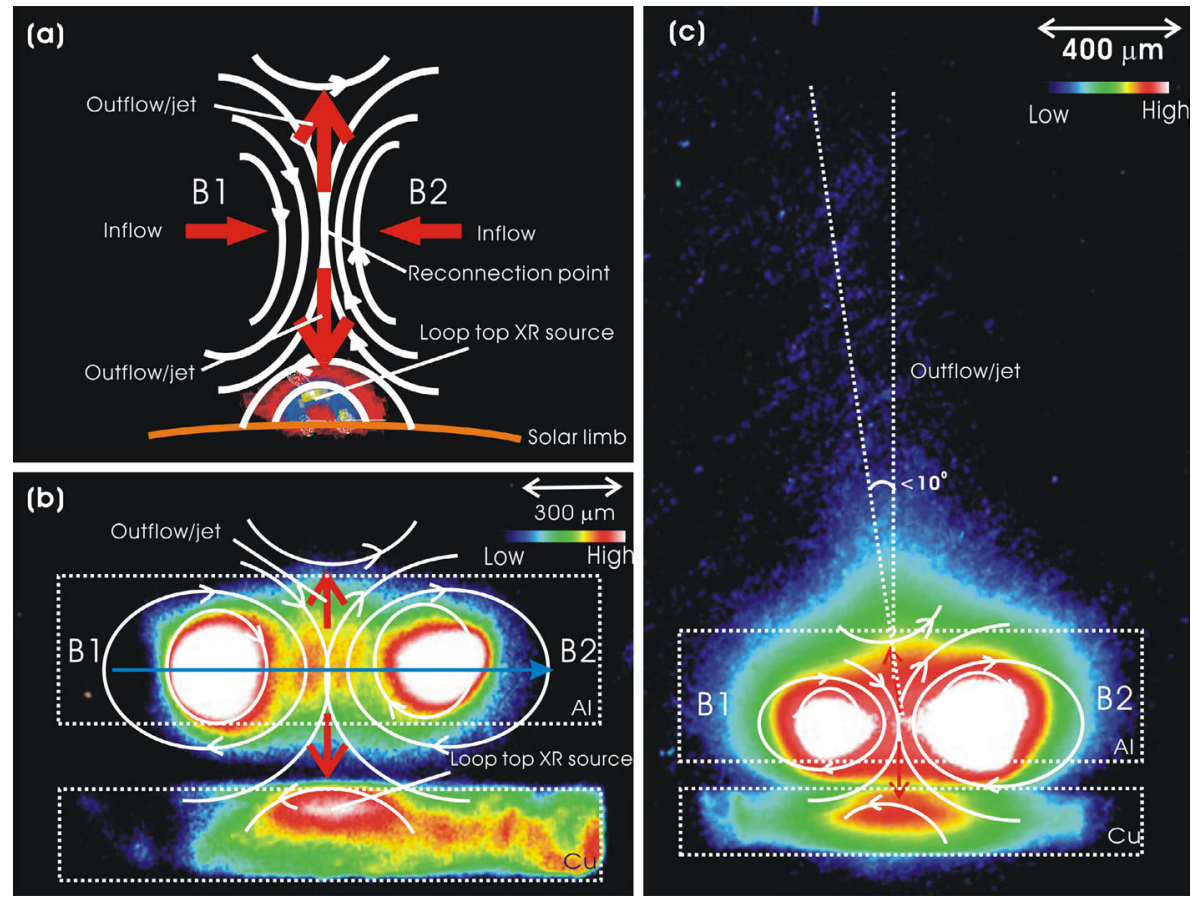

Fig. 15 Loop-top-like X-ray source and outflows observed in the laboratory. (a) Magnetic reconnection model for the loop-top X-ray source in an eruptive solar flare, with a sketch depicting the X-ray observation scheme of Masuda et al. (1994). (b) The pinhole X-ray image observed forward of the Al foil target. Magnetic field lines are illustrated based on the flux surface of the plasma bubbles. The $\mathrm{Al}$ and $\mathrm{Cu}$ targets are the rectangles enclosed by white dotted lines. The red arrows indicate outflow/jet directions. (c) X-ray image with two laser spots separated by $400 \mu \mathrm{m}$ and with a foil thickness of $10 \mu \mathrm{m}$. The asymmetry of the laser intensity on the Al target causes an imbalance of the laser spots as well as of the magnetic fields B1 and B2, and further induces the inclination of the upward outflow. The downward outflow impinges on the $\mathrm{Cu}$ target and results in a hot X-ray source. From Zhong et al. (2010)

(2010) could be treated as a plasmoid. Although a hot X-ray spot and a plasmoid are produced in different ways in different environments, they are both the magnetized plasma blobs, and magnetic reconnection could take place when a hot spot (plasmoid) approaches to another spot (plasmoid). This indicates that the results of Zhong et al. (2010) confirmed from the point of view of the laboratory experiment that the coalescence of plasmoids in the CS causes the secondary magnetic diffusion, which partly explains why fast magnetic reconnection could still occur in a thickness CS during the major eruption.

Figure $15 \mathrm{~b}$ also shows the existence of the outflow jets with an opening angle of $10^{\circ}$, leading to a feature becoming broader with increasing distance from the center of the CS, which is similar to what is observed for CS on the Sun. We note that the scale of the system in lab is only a few $\mathrm{mm}$, so we may not necessarily be able to expect a very long CS in such a system. On the other hand, regarding the scales of the reconnection process occurring in different environments, the magnetic configuration shown in Fig. 15 has a scale of about $2 \mathrm{~mm}$, and the CS is about $0.3 \mathrm{~mm}$ in length. According to Table 1 of Zhong et al. (2010), which displays the similarity of solar flares to laser-produced plasmas, $1 \mathrm{~mm}$ in lab could be equivalent to up to $10^{10} \mathrm{~cm}$ on the Sun, therefore the CS of $0.3 \mathrm{~mm}$ long in lab could be scaled to $3 \times 10^{9} \mathrm{~cm}$ on the Sun, which may not be considered short. 


\section{Internal Structures and Thickness of Current Sheets Created in Solar Eruptions}

The high electrical conductivity and force-free environment in the corona confine the reconnection region to a thin layer, so a long and thin current sheet is expected to develop in major eruptive processes. Such features of the reconnecting current sheet allow us to treat the current sheet as an infinitely thin line when the main purpose is to study the global behavior of the eruption (e.g., Lin and Forbes 2000). Although indirect evidence indicates the existence of the current sheet in the eruption (e.g., see Forbes and Acton 1996 for a brief review), direct observation of a thin current sheet is difficult. This is because both size and emission of the current sheet are easily dominated by other large-scale structures nearby (e.g., see Ko et al. 2003 for detailed discussions).

It is often assumed that the current sheet is too thin to be observable since its thickness, $d$, is believed to be limited by the proton Larmor radius (Litvinenko 1996; Wood and Neukirch 2005; and references therein), which is tens of meters in the coronal environment. This view is based on information about small-scale magnetic reconnection in laboratory (with the size of meters). When the sheet forms in the solar eruption, on the other hand, it could develop in length at speed of a few hundred $\mathrm{km} \mathrm{s}^{-1}$ (Forbes and Lin 2000; Lin and Forbes 2000; Lin 2002). In such a highly dynamical process, the Larmor radius of particles cannot govern the scale of the magnetic structure. Instead various plasma instabilities will likely occur and play an important role in diffusing magnetic field and in governing the scale of the sheet (Strauss 1986, 1988).

Lin et al. (2007) analyzed a set of unique data for three eruptions observed by UVCS and LASCO. They found that, in some circumstances, the current sheets are observable, and their thickness in real events may range from $10^{4}$ to $10^{5} \mathrm{~km}$ ! This led to a very large effective electric resistivity, $\eta_{e}$, of the plasma inside the current sheet, which could reach up to $5 \times 10^{5} \mathrm{ohms} \mathrm{m}$, about $12-13$ orders of magnitude larger than Spitzer's classical resistivity in the quiet corona, and 4-5 orders of magnitude larger than the anomalous resistivity that is due to interactions between electrons and low frequency ion-acoustic turbulence (e.g., see Priest 1982, pp. 80-81).

These results imply that large-scale plasma turbulence operates within the sheets during the eruption, and poses a serious challenge to the existing understanding and theories of reconnection and particle acceleration in the sheet. In sections below, we discuss observations and consequent results of the thickness of the CS, and relations of these results to the theories mentioned above.

\subsection{Observed Apparent Thickness of Current Sheets}

As we mentioned earlier, the dark Ly $\alpha$ gap observed in the 2003 November 18 event marks the reconnection outflow region, which is close to the inflow region. So the decrease in the width of the dark gap in Ly $\alpha$ images displayed by Fig. 7 means the shrinkage of the outflow region in size, and depicts the reconnection inflow outside the CS as well. In addition, the plot also shows the asymptotic feature, which suggests the ceasing of the reconnection inflow at the edge of the current sheet. Therefore, we can deduce the apparent thickness, $d$, of the sheet according to the tendency of the curve, and obtain $d=6.4 \times 10^{4} \mathrm{~km}$. Because of the projection effects, this value is considered the upper limit to the actual value of $d$ (see also discussions of Lin et al. 2007 in detail).

Similarly, we are also capable of deducing the apparent value of $d$ via studying the image of the UVCS slit in [Fe XVIII]. As shown by Fig. 15 of Ko et al. (2003), Figs. 1 and 2 of 
Fig. 16 Distributions of the [Fe XVIII] intensity along the UVCS slit in two time intervals during the event occurring in the period from January 8, 2002 to January 11, 2002. Upper panel: 20:46 UT to $23: 19$ UT on January 10, and lower panel: 23:21 UT on January 10 to $03: 18$ UT on January 11. Histograms in both panels are the observational data, and the dotted curves are Gaussian profiles fitted to the data. The apparent value of $d$ is determined by the FWHM of the Gaussian profile. From Lin et al. (2009)
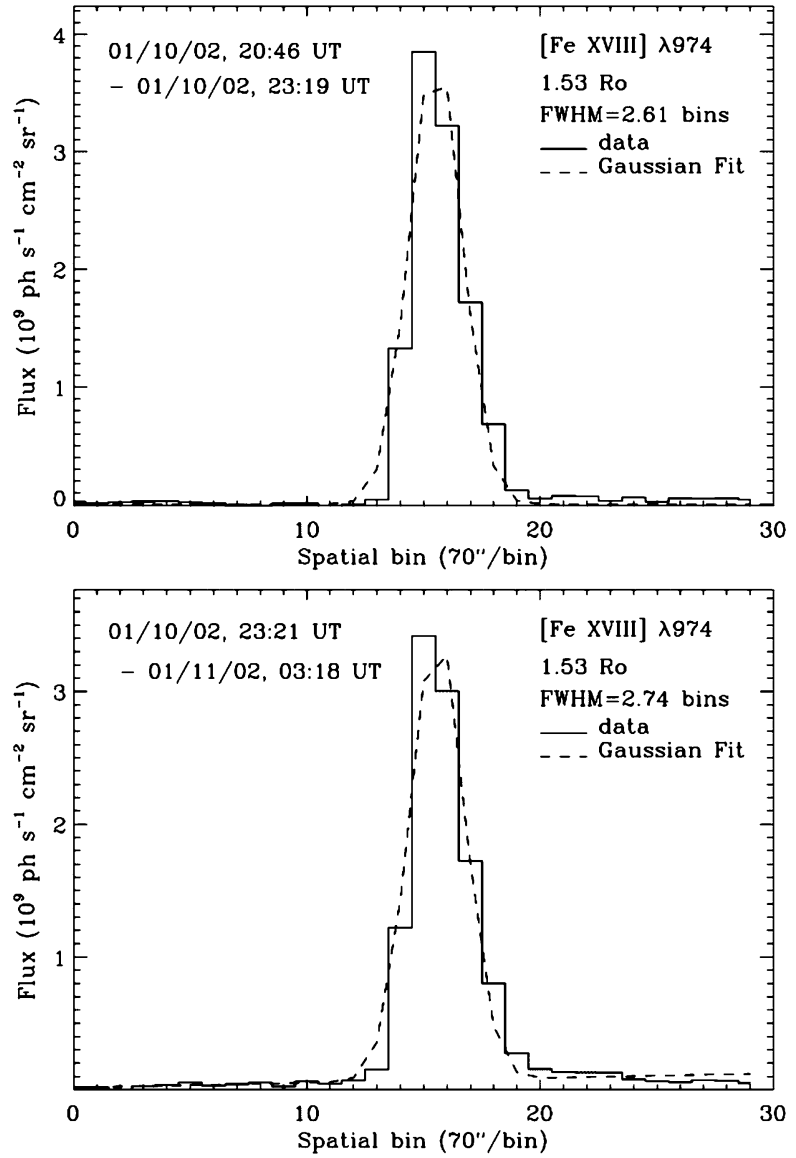

Ciaravella and Raymond (2008), and Fig. 4 of this work, the bright spots in [Fe XVIII] on the UVCS slit approximately showed the location and the extent of the CS in space because the occurrence of [Fe XVIII] emission indicates heating as a result of reconnection that is taking place or has taken place in the CS. Plot the intensity of the [Fe XVIII] bright spot along the slit, we obtain the full-width at half-maximum (FWHM) of the intensity distribution, which in fact gives the apparent value of $d$. Applying this method to the intensity of [Fe XVIII] emission shown in Fig. 4, Lin et al. (2009) obtained $d=1.1 \times 10^{5} \mathrm{~km}$ for the event of 21 March 1998. Figure 16 showed two distributions of the [Fe XVIII] intensity along the UVCS slit in two time intervals in the event occurring in the period between January 8, 2002 and January 11, 2002. Histograms in both panels are the observational data, and the dotted curves are Gaussian profiles fitted to the data. The apparent value of $d$ is determined according to the FWHM of the Gaussian profile, which gives $d=1.3 \times 10^{5} \mathrm{~km}$.

We may need to note here that the possibility exists that the apparent value of $d$ seen in [Fe XVIII] might contain the component contributed by a so-called "thermal halo" around the CS. Numerical experiments performed by Yokoyama and Shibata $(1998,2001)$ indicated that if the heat conduction was included in the simulation, the thermal energy inside the CS could spread out of the CS because of the difference in temperature between the CS and the inflow region, heating the plasma outside the CS and forming a thermal halo around the CS. Seaton and Forbes (2009) and Reeves et al. (2010) confirmed this result in the numerical 
experiments with higher resolution and in the spherically symmetric configuration. Spreading of the thermal energy could result in a CS embedded in the thermal halo, and what we would observe directly is actually the halo and the apparent value of $d$ is in fact the scale of the halo instead of the CS. We shall discuss this issue in detail later.

The CS is also often observed by LASCO on SOHO, studying the LASCO white-light images may also reveal the information of apparent $d$. In addition to analyzing the UVCS data, Lin et al. (2009) also investigated the size of the current sheet developed in the above two events via studying the LASCO/C2 and C3 data by an approach known as limb synoptic maps (LSMs; Li et al. 2000). An LSM is made by extracting a strip around the solar limb at a fixed altitude from an image, and then aligning the strips in time sequences. It displays the position and/or displacement of an object in polar angle (PA) at various altitudes and times, helps determine the scale (especially $d$ ) of some smaller features like the current sheets in a simple and straightforward fashion, and makes it easy to look into the variation of $d$ in space. In this approach, the intensity distribution of the current sheet along the strip is first displayed, and then the apparent value of $d$ is determined by the FWHM of the Gaussian profile used to fit the observational distribution. Lin et al. (2009) found that analyzing the LASCO data by LSMs still brought the apparent value of $d$ to the range from $10^{4}$ to $10^{5} \mathrm{~km}$.

When studying the event of 26 June 2005 and performing the relevant plasma diagnoses, Vršnak et al. (2009) also re-investigated the LASCO white-light data of the events of 8 January 2002 and 18 November 2003. They noticed that the current sheet could be wavy and be of multi-ray-like features, which implied complex internal structures of the CME/flare current sheet. Their results confirmed that the apparent value of $d$ could be between a few $10^{4}$ and a few $10^{5} \mathrm{~km}$.

In addition to plasma flows inside the current sheet in the wake of the cart-wheel CME, Savage et al. (2010) measured the apparent thickness of the current sheet (see Fig. 13), and found that $d \leq 4 \times 10^{3} \mathrm{~km}$. Re-studying the same event, Landi et al. (2012) later measured $d$ again, and obtained that $d$ was at least $1.3 \times 10^{5} \mathrm{~km}$. It might be difficult in judging which result is more reliable than another one, but it is clear that both values are huge compared to the skin depth of the coronal plasma. This means the properties of the CME/flare CS that always involves in a highly dynamic fashion are governed by the global behaviors of the large scale structures nearby and the associated dynamical evolution. We may also note that Savage et al. (2010) and Landi et al. (2012) used different data to study the scale of the CS, the former used the HINODE/XRT data and the latter used HINODE/EIS data. Therefore, the thermal halo effect could probably play a role in governing the apparent values of $d$.

Most recently, Ling et al. (2014) studied the current sheet formed in the eruption of 7 September 2005. The source region of this event was active region AR 10808, which was likely associated with a number of fast halo CMEs from late August to September 5. When it was on the visible disk, this region produced $25 \mathrm{M}$-class, and $10 \mathrm{X}$-class flares over ten days. The eruption of 7 September 2005 produced the last extremely energetic flare of solar cycle 23 (optical class 3B, X-ray class $>17$, saturating GOES instrument), which led to a severe radio blackout affecting communication and navigation systems, and a fast CME at speed of about $2500 \mathrm{~km} \mathrm{~s}^{-1}$, which was the fastest ever observed by MK4. As expected, the event developed a long current sheet connecting the CME to the associated flare.

This event is probably the first one of which only the data obtained by the ground-based instrument were available for studying the CME/flare CS because SOHO was not operating during the event. What is nice about the work of Ling et al. (2014) is that they used data obtained by the MLSO K-coronameters, which have been continuously improved in resolution at low altitudes, and the radius of the occulting disk has been continuously decreased; therefore the FOV of the present MK4 extends from 1.08 to $2.86 \mathrm{R}_{\odot}$ in heliocentrical distance, 

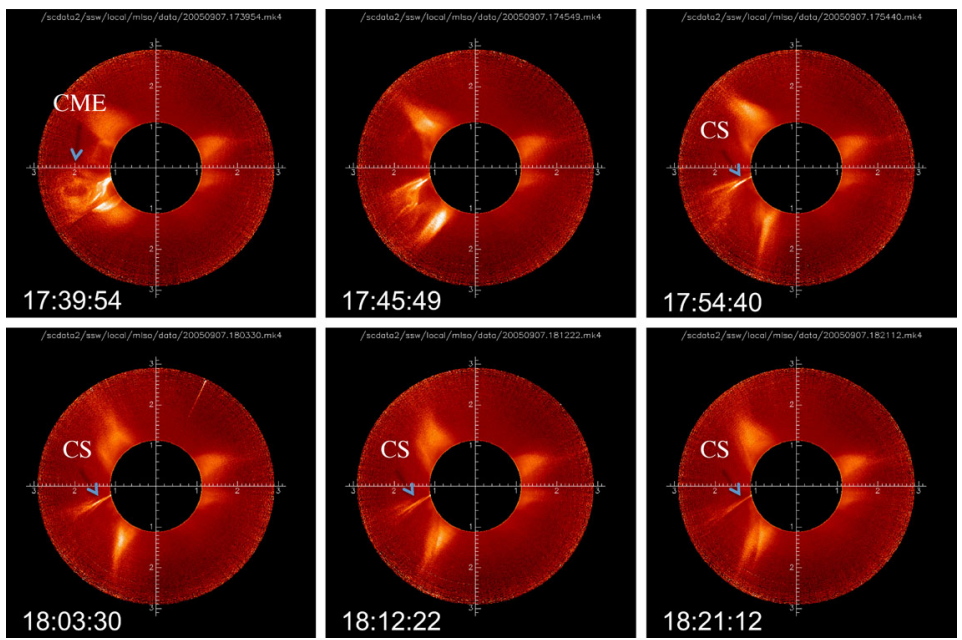

Fig. 17 MK4 white light vignetted data in rectangular coordinates showing the progression of the CME and its trailing ray on 2005 September 7. The observation times (UT) are indicated on the images. CME and CS are specified in each panel. From Ling et al. (2014)

which allows us to observe the objects in the very low corona in white-light. Usually, the region below $1.5 \mathrm{R}_{\odot}$ is very important for investigating the connection of the current sheet to the flare loop system as well as for looking into the lower part of the sheet itself since the high temperature flare loops and the associated cusp region in a typical eruptive process often appear above the altitudes $\geq 4 \times 10^{4} \mathrm{~km}$ (e.g., see Lin 2002 and references therein).

Observations of the event on September 7, 2005 by MK4 provided a very good opportunity for studying the early evolution of the current sheet at low altitudes (Fig. 17), including the formation and development as well as physical properties of the current sheet although only white-light data are available. Furthermore, the small radius of the occulting disk of MK4 even allows us to see the lower tip of the CS in the FOV of the coronagraph. Consistent with indications by Table 2 that the flare loops in the major eruption could usually exceed the height from the solar surface up to $0.14 \mathrm{R}_{\odot}$, the lower tip of the CS developed by this event indeed appeared in the MK4 FOV.

From the MK4 data, it is straightforward to determine the apparent value of $d$ by directly measuring the FWHM of the brightness distribution of the sheet in the direction perpendicular to the sheet extension, and $d$ as a function of the distance from the solar center could be further obtained. Results of Ling et al. (2014) showed that $d$ varied with both distance and time such that the smallest value of $d$ was obtained near the sheet base, and widened with height and time. After the measurement became possible, the narrowest region of the sheet had the width of $\sim 7 \times 10^{3} \mathrm{~km}$, which was measured near the base of the sheet, and $d$ had the typical value of $2.8 \times 10^{4} \mathrm{~km}$ at most locations on the CS. This result is consistent with those deduced from the LASCO data that the apparent width of the CS becomes larger with height and time. We need also note here that, on the other hand, in the framework of the traditional Petschek-type reconnection, the jet of the reconnected plasma from the diffusion region may become wider at higher altitudes (cf. Fig. 15). In this sense, the feature in the region that was observed to increase in the width might not be the CS, but a collimated plasma jet.

Before we end this section, it is interesting to note that the value of $d$ in different cases observed by various instruments does not differ from one another very much. Observations 
by LASCO and UV spectroscopy gave the similar results for $d$ in the lower corona ranging from $6.4 \times 10^{4}$ to $2.1 \times 10^{5} \mathrm{~km}$, and this value almost doubled at higher altitudes of $4-5 \mathrm{R}_{\odot}$. At even lower altitudes, say between 1.1 and $1.2 \mathrm{R}_{\odot}$, it is remarkable that observations of the ground-based instrument (for example, MK4) and those of the space-borne instruments (for example, Hinode/XRT) indicated that the width of the white-light current sheet $(7.0 \times$ $\left.10^{3} \mathrm{~km}\right)$ and that of the X-ray current sheet $\left(4-5 \times 10^{3} \mathrm{~km}\right.$, see also Savage et al. 2010) possessed almost the same apparent values at similar or slightly different altitudes, but the associated CMEs had quite different speeds $\left(2.5 \times 10^{3} \mathrm{~km} \mathrm{~s}^{-1}\right.$ compared to $\left.450 \mathrm{~km} \mathrm{~s}^{-1}\right)$.

\subsection{Impact of Projection Effects on Apparent Values of $d$}

Because of projection effects and possible complex morphology of the current sheet and the associated magnetic field, the above values for $d$ are upper limits of the true values of $d$. Suppose the current sheet is a plate of true thickness $d$ and true depth $D$, and LOS is in the $x$-direction (Fig. 18). Generally, the emission measure (EM) of the CS observed in a given spectral line is related to the density inside the CS, $n_{e}$, and the path length in CS along LOS, $D^{\prime}$, in the way of $n_{e}^{2} D^{\prime}$; and the brightness of white-light images or that of the scattered Ly $\alpha$ lines, $I_{s c}$ is proportional to $n_{e} D^{\prime}$. As a result of the modest EM of the current sheets in eruptive events compared to that of the nearby corona, on the other hand, the current sheet may be faint and difficult to detect if it is not observed roughly edge-on. Below, we discuss how projection effects impact measurements of $d$ (see also Lin et al. 2009).

All the images we analyzed above are projections of the emission from an optically thin three-dimensional structure onto the two-dimensional plane of a detector. Since the intensity recorded by the detector is an integration of the total emission along LOS, the intensity level is related to both the density and the column depth along LOS. Therefore, viewing a bright object at various angles may yield different impressions (see detailed discussions of Forbes and Acton 1996). For a current sheet of given $d$ and extent in other dimensions, seeing it edge-on results largest integral path and thus the largest EM, and seeing it face-on, on the other hand, results in the shortest path and the lowest EM.

In the case of a bright sheet appearing in the dark background, as in the LASCO images, the brightness, $b$, of the sheet is linearly dependent on the length of LOS inside the sheet. For simplicity, the plasma density in the background is set to be zero. If the current sheet is observed edge-on (Fig. 18a) and LOS is parallel to the long axis of the sheet, the observed thickness of the sheet is its true thickness, $d$, and its brightness (or the EM) $b$ is proportional to the path along LOS in the sheet $s=D$, namely $b \propto s$. If the current sheet is tilted by an angle $\theta$, the apparent sheet thickness becomes $d^{\prime}$ and the path along LOS becomes $s=D^{\prime}$ (Figs. 18b and 18c).

Lin et al. (2009) noticed that, suppose the uniform distribution of the mass inside the sheet, in the case of small $\theta$ such that $\tan \theta \ll d / D$ (Figs. 18b), $d^{\prime}$ is related to $d$ in the way of $d^{\prime} / d \leq 2$, which indicates that the increase in the apparent thickness caused by the projection effects is within the factor of 2 . With increase in $\theta$, the apparent brightness of the sheet, $b^{\prime}$, is related to $b, d$, and $\theta$ such as $b^{\prime} / b=\left(d^{\prime} / d-\cos \theta\right)^{-1}$. Here, $d^{\prime} / d$ is always larger than unity, and $\cos \theta$ is between 0 and 1 . So the impact of $\cos \theta$ could be ignored as $d^{\prime} / d$ gets big enough, and the brightness of the sheet is roughly inversely proportional to the observed thickness of the sheet. This implies only a limited impact of projection effects on measuring $d$.

In reality, direct observations of the current sheet are also affected in an important way by the signal-to-noise ratio of instruments themselves in addition to being affected by brighter structures nearby. Combining these effects leads to that the current sheet becomes hard to be 
Fig. 18 Schematic diagram of orientations of the sheet and the corresponding parameters for the sheet properties. (a) The sheet is observed edge-on; (b) the sheet is observed at a small angle; and (c) the sheet is observed at a large angle. From Lin et al. (2009)

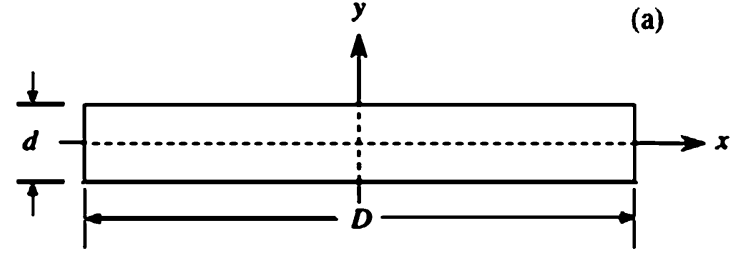

(b)

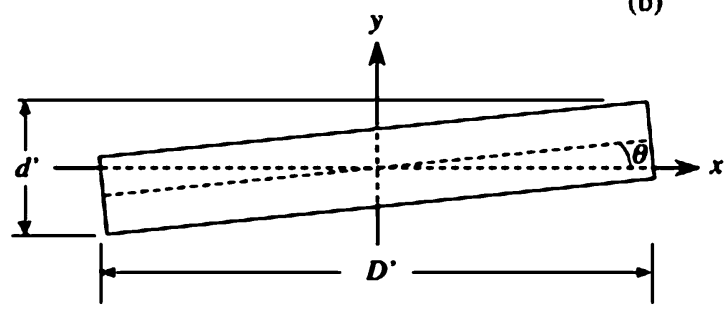

(c)

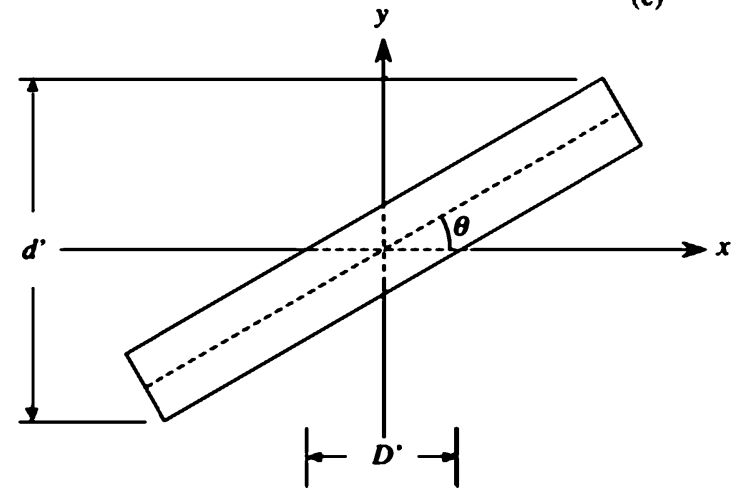

identified as $d^{\prime} / d$ exceeds 10 (see also Forbes and Acton 1996). According to the relation of EM and $I_{c s}$ to $n_{e}$ and $D^{\prime}: \mathrm{EM} \sim n_{e}^{2} D^{\prime}, I_{c s} \sim n_{e} D^{\prime}$, we have $n_{e} \sim \mathrm{EM} / I_{s c}$ and $D^{\prime} \sim I_{s c}^{2} / \mathrm{EM}$. With $D^{\prime}$ deduced this way, $d^{\prime}$ could be measured directly from observations. Comparing $D^{\prime}$ and $d^{\prime}$ could help us evaluate the impact of the projection effects. Consulting Fig. 18, if the projection effects could be ignored, $d^{\prime}$ should be small compared to $D^{\prime}$ (Figs. 18a and 18b); and a serious consideration of projection effects is needed if $d^{\prime}$ and $D^{\prime}$ are comparable to each other (Fig. 18c). Using this method, Ciaravella and Raymond (2008) found that $d^{\prime} / d$ is between 2.5 and 5 for the current sheet developed in the eruption of 2003 November 4 .

In addition to the case of planar CS as shown in Fig. 18, the CS in reality may be very likely to have a wavy configuration (see Fig. 2 of Vršnak et al. 2009, which depicted a CS that looks very like a curtain) as well. In this case, the complex internal structure could account for "multi-ray" features of the post-CME CS that were also often observed (see Ko et al. 2003; Vršnak et al. 2009; Ciaravella and Raymond 2008; Ling et al. 2014), and brings more uncertainties in determining the geometric size of the CS in a specific case since it is hard to tell how many folders a wavy CS could have. So it is worth investing more effort in investigating this issue in the future. Such effort is two-fold: enhancing the resolution of observations in multiple wavelengths so that fine structures in the CS could be well recognized and studied, and performing the corresponding numerical experiments so that we could understand the physics governing manifestations of the CS. 


\subsection{Reasonableness of Large Values of CS Thickness and Resistivity}

Results and discussions given above indicated that a CS developed in the solar eruptive events is recognizable and the apparent value of $d$ could be easily obtained when the plane in which the CS extends is roughly parallel to LOS, namely it is observed edge-on. Because it is difficult to recognize a CS and to measure its thickness as the CS is observed face-on, most of the CSs we have recognized and studied were observed edge-on although recognizing a face-on CS is much less difficult in the SDO era (e.g., see Warren et al. 2011; Savage et al. 2012a, 2012b; McKenzie 2013; Doschek et al. 2014; Hanneman and Reeves 2014).

The existing observations and the related results confirm that the apparent values of $d$ could be up to $10^{3}-10^{5} \mathrm{~km}$ although these observations were made for different events by different instruments, and impact of the projection effects on the measurements turned out to be limited. But our expectation is to completely remove this impact in order to get the true values of various observables. For the time being, on the other hand, it is impossible to remove technically the impact of the projection effects completely on the apparent values of observables although observations by STEREO could help partly resolve this problem (e.g., see Isavnin et al. 2013 and references therein). To reach a more meaningful conclusion about the true value of the CS thickness, the corresponding lower limits need to be figured out by combining the well-known theory and observations.

Forbes and Malherbe (1991), Y. Fan (2005, private communication), and Riley et al. (2007) suggested approaching this problem by interpreting the plasma blobs flowing along a CS as the magnetic islands resulting from the tearing mode. According to our previous discussions, we realized that those blobs of various sizes appearing in the reconnecting CS are very likely to be the products of different types of plasma instabilities and the consequent turbulence, as well as the resultant small structures (see Kliem et al. 2000; Shibata and Tanuma 2001; Bárta et al. 2011a; Shen et al. 2011; and references therein). The existence of internal small structures of the CS could be indirectly indicated by the location of the HXR source region above the flare loop (Sui et al. 2006), or by the sub-second pulse of the HXR emission (see Aschwanden 2004 and references therein) as well as the time-profile of the quasi-periodic reversals of radio polarization at $17 \mathrm{GHz}$ (Huang and Lin 2006). These results suggest that particle acceleration occurs inside reconnecting CS. Plasma instabilities and the associated consequences could be one source of these structures, and the inhomogeneities of plasma and magnetic field in both space and time during the reconnection process could be another one. Interested readers are referred to Kliem et al. (2000), Shibata and Tanuma (2001), Lin et al. (2006), Liu et al. (2010), and so on.

The tearing mode instability is probably the mode of the most importance of the various possible instabilities that can act in the CS (see discussions of Priest 1988; Priest and Forbes 2000, pp. 177-185). The other possible instabilities that may occur in a structure like CS are gravitational and rippling modes instabilities. The former exists when a gravitational (or equivalent) force acts transverse the CS to produce a density stratification, and the latter takes place as the magnetic diffusivity is non-uniform across the CS that is usually caused by the temperature structure in the CS. As showed by Furth et al. (1963), the tearing mode could be excited as long as the resistivity exists and the aspect ratio (namely the length divided by the thickness) of the CS exceeds $\sim 2 \pi$, without requiring gravitational and resistivity gradients. The tearing mode drives turbulence leading to the release of magnetic energy and reorganization of the magnetic field after topology changes. Because reconnection in the corona is often patchy (e.g., Guidoni and Longcope 2011), the turbulence is likely to be intermittent. The tearing mode and the resulting turbulence largely govern both the geometric features of the CS and many of the important properties of magnetic reconnection. We are able to deduce the lower limit of the CS thickness by relating 
Fig. 19 Interior structure of the current sheet in which the tearing mode instability develops. Thick arrows show plasma flow, and thin arrows are for magnetic field lines. (Courtesy of E.R. Priest)

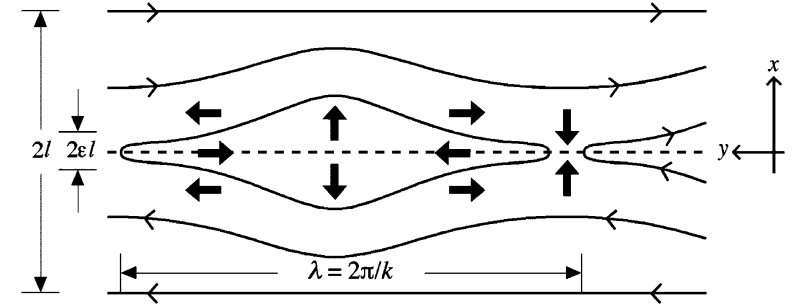

observational consequences of the turbulence due to the tearing mode to the corresponding theories.

Long current sheets can easily become unstable to the tearing mode. This is shown by both theories (e.g., Furth et al. 1963) and numerical experiments (Ambrosiano et al. 1988). In two dimensions, the current sheet is torn into many small-scale magnetic islands (see Figs. 6.1 and 6.3 of Priest and Forbes 2000, which described possible modes of instabilities that may develop in the CS, and more detailed features of a torn CS, respectively). In three dimensions, the current sheet develops flux ropes which are the counterpart of twodimensional magnetic islands (compare to Daughton et al. 2011). The turbulence developing from the tearing mode enhances diffusion of the large-scale magnetic field. The turbulence converts part of the magnetic energy into the kinetic energy of the perturbation, which in turn creates perturbed small-scale fields via the dynamo process (Bhattacharjee and Yuan 1995). The growth of the perturbed field is also governed by the tearing mode (see Strauss 1986, 1988), and small-scale field stochasticity and braiding produced in this process yields an effective perpendicular momentum transport, which leads to an anomalous electron viscosity (see also discussions of Biskamp 1993, p. 22). This results in a double diffusive process and causes more efficient diffusion in the reconnecting current sheet. If the process operates in a CME CS, then there is likely to be reasonably fast reconnection even in a fairly thick CS.

When the tearing mode develops (Fig. 19) with the growth rate slower than the hydromagnetic rate but faster than the resistive diffusion rate, its wavenumber $k$ is related to $l$ such that $S^{-1 / 4}<k l<1$ (e.g., see Furth et al. 1963), where $l$ is the half thickness of the CS (namely $d=2 l$, referring to Fig. 19), $S=\tau_{d} / \tau_{A}$ is the Lundquist number of the CS, and $\tau_{A}=l / v_{A}$ and $\tau_{d}=l^{2} / \eta$ are the times at which the Alfvén wave and the resistive diffusion traverse the CS, respectively. We note here that $S$ is the local Lundquist number of the CS, not the global one of the whole system, and $V_{A}$ is the local Alfvén speed near the CS. Therefore, $S=\tau_{d} / \tau_{A}=v_{A} l / \eta=v_{A} / v_{i}=1 / M_{A}$ according to the standard theory of magnetic reconnection (see Priest and Forbes 2000).

If the plasma in the CS has sufficiently high conductivity such that both $S$ and $S^{1 / 4}$ are large compared to unity, magnetic dissipation or reconnection will be confined to a very thin CS and the tearing mode develops with very long wavelengths (e.g., see Furth et al. 1963; Priest and Forbes 2000). In this case, $k l \ll 1$ holds so that the turbulence could grow at the rate between $1 / \tau_{d}$ (the resistive diffusion rate) and $1 / \tau_{A}$ (the hydromagnetic rate). For the events so far observed to take place in the solar atmosphere, on the other hand, $M_{A}(S)$ varies from $10^{-3}\left(10^{3}\right)$ to $10^{-1}$ (10) (e.g., Yokoyama et al. 2001; Ko et al. 2003; Lin et al. 2005; Liu et al. 2010; Savage et al. 2010). So although $S \gg 1$ did hold for these events, $S^{1 / 4} \gg 1$ did not. Therefore, $k l$ possesses a lower limit of finite value, providing us an approach to determining the lower limit to the apparent value of $d$ such that 


$$
S^{-1 / 4}<k l, \quad l>M_{A}^{1 / 4} / k, \quad l_{\min }=\lambda M_{A}^{1 / 4} /(2 \pi),
$$

where $\lambda=2 \pi / k$ is the wavelength of the turbulence.

We may also note here that Loureiro et al. (2007) analyzed the relation of $l$ to the Lundquist number in a different way. They investigated the dependence of $k l$ on the global Lundquist number, $S_{L}$, for the whole system instead of the local one, $S$, for the CS, as the rate of reconnection reached maximum. They started with a CS configuration described by the Dawson function, and found that at the maximum reconnection rate, $k l \sim S_{L}^{-1 / 8}$. If the plasma inside the CS undergoes turbulence in a more general sense, the minimum rate of reconnection would depend on $S_{L}$ in a little more significant way, $M_{A} \sim S_{L}^{-3 / 16}$ (see Lazarian and Vishniac 1999), and $k l$ is roughly proportional to the speed of plasma blobs compared to the local $v_{A}$ (e.g., see also Lazarian et al. 2014 and references therein), which is a factor of around 3 less than $M_{A}$ according to Shen et al. (2013). This means that $k l \sim S_{L}^{-3 / 16}$ in the turbulent reconnection process. We see that $S_{L}$ could be as large as $10^{12}$, but $S_{L}^{1 / 8} \approx 31.6$ and $S_{L}^{3 / 16} \approx 178$, which are not very large, and the corresponding value of $l$ may not be very different from that of $l$ determined by (1). Later, Bhattacharjee et al. (2009) studied the reconnection process starting from a Harris sheet, and brought the dependence of $k l$ on $S$ to $k l \sim S^{-1 / 4}$ as the fastest reconnection took place. Therefore, the possible values of $l$ governed by scaling laws for the turbulent reconnection have roughly the same order of magnitude no matter how the process is initiated. More discussions about the dependence of features and properties on $S$ and/or $S_{L}$ manifested by the turbulent reconnection can be found in the introduction of Murphy et al. (2013) as a brief review.

With $M_{A}$ and $\lambda$ given, we are able to estimate $l_{\min }$ according to (1). As suggested by Ko et al. (2003) and Lin et al. (2005), the plasma blobs observed to flow along the CS were identified with the magnetic islands (turbulent eddies) resulted from the tearing mode instability and the consequent turbulence, so the value of $\lambda$ in Eq. (1) could be identified with the distance between the two adjacent blobs (see also Fig. 10). Usually, the value of $M_{A}$ could be obtained via different approaches (see also Yokoyama et al. 2001; Ciaravella et al. 2002; Ko et al. 2003; Lin et al. 2005; Bemporad et al. 2006; Ciaravella and Raymond 2008; Savage et al. 2010; Liu et al. 2010, 2013; Liu 2013; Su et al. 2013), and then estimate $l_{\text {min }}$ according to (1) is straightforward. For example, the minimum of the distances of every two adjacent blobs observed in the 2002 January 8 event is $3.062 \times 10^{5} \mathrm{~km}$ (see Fig. 10), namely $\lambda=3.062 \times 10^{5} \mathrm{~km}$, and $M_{A}$ ranged from 0.01 and 0.03 (Ko et al. 2003). Taking $M_{A}=0.01$ gives $l_{\min }=1.7 \times 10^{4} \mathrm{~km}$ and $d_{\min }=3.4 \times 10^{4} \mathrm{~km}$, which is different from $d_{\max }=1.5 \times 10^{5} \mathrm{~km}$ by a factor of 4 . This is consistent with the result of Ciaravella and Raymond (2008).

Further investigations indicated that a more general expression relating $l_{\min }$ to $M_{A}$ and $\lambda$ exists such that

$$
l_{\min }=M_{A}^{\alpha} \lambda /(2 \pi),
$$

for different boundary conditions and different modes of the tearing (for instance, double or even multiple tearing, Pritchett et al. 1980; Priest 1985). Equation (2) is generally valid for different cases with $\alpha$ being a constant between $1 / 7$ and 1 (see also Loureiro et al. 2007; Lin et al. 2009). Taking $\alpha=1 / 7$ for the instability in a CS with a periodic internal structure (see also Bobrova and Syrovatskii 1980 for more details), we have larger $l_{\min }$ than the above results; taking $\alpha=1 / 4$ for the "constant- $\psi$ " approximation (namely fluctuations perpendicular to the CS are small) results in the scaling law given in Eq. (1); and taking $\alpha=1$ for the case that the "constant- $\psi$ " approximation breaks down (see also Pritchett et al. 1980; Priest 1985) brings $l_{\min }$ to the range from $7.4 \times 10^{2}$ to $4.55 \times 10^{3} \mathrm{~km}$, which is 
$10 \sim 100$ times smaller than the apparent values of $l$. This difference is surely larger than 4 that we have just mentioned shortly, but apparently much less than the difference between $10^{4} \mathrm{~km}$ and a few meters or a few $\mathrm{km}$ ! This indicates that the true value of $d$ should not differ very much from the apparent value as long as the CS could be recognized in observations.

Before ending this part of work, we point out that many authors have inferred observationally the presence of turbulence inside the post-CME CS, which could account for the large $d$ and $\eta_{e}$, from the detection of significant non-thermal line broadening in EUV/UV spectral lines. For instance, Ciaravella et al. (2002) deduced that the turbulent motion had speed less than $60 \mathrm{~km} \mathrm{~s}^{-1}$ in the CS (see also discussions of Kohl et al. 2006); Ciaravella and Raymond (2008) measured [Fe XVIII] line widths in a CS that were $100 \mathrm{~km} \mathrm{~s}^{-1}$ larger than the thermal widths as a result of a combination of bulk motions and turbulence. The Petschek-type and turbulent reconnection processes must work together to account for observations (e.g., see also discussions of Mei et al. 2012 from a theoretical view point). Susino et al. (2013) noticed significant non-thermal broadening in the CS as a result turbulent motions when analyzing the EUV data from UVCS. Bemporad (2008) further pointed out that the post-CME current sheet is an assembly of multiple small-scale structures, which result from the turbulence and could be responsible for both high temperature and large thickness of the CS. Doschek et al. (2014) found 20-60 $\mathrm{km} \mathrm{s}^{-1}$ non-thermal motions with Fe XXIII and Fe XXIV lines above flare loop in EIS spectra.

\section{CS and Its Internal Fine Structures Observed in Numerical Experiments}

Numerical simulations and experiments provide us a very important and practical approach to studying these instabilities and their impact on the magnetic reconnection. There have been a large number of works and results of magnetic reconnection in the fields of plasma physics, geophysics and space sciences, stellar and interstellar physics, and interested readers are referred to the books by Priest and Forbes (2000) and by Biskamp (2000), or works of Shay et al. (2011) and Opher et al. (2011), or reviews by Cassak and Shay (2012) and Lazarian et al. (2014). We are focusing in the present work on reconnection occurring in the CS during solar eruptions, as well as the physical properties of magnetic reconnection and the CS revealed by these results.

An inevitable question related to our discussions in previous sections is: How can fast magnetic reconnection take place in such a thick CS? A leading explanation is that the occurrence of the tearing mode in the CS results in turbulence and that leads to small scale structure that enhances the diffusion of the magnetic field, and thus allows the magnetic energy to be converted into heat and kinetic energy at a reasonably fast rate in a thick CS. The question following this is then in a real solar eruptive process, what is the smallest size of these fine structures so that the energy conversion could be considered fast? Ichimaru (1975) noted long ago that a basic property of magnetic reconnection is that it is turbulent, and a reconnecting CS should contain a wide spectrum of structures at various sizes. Shibata and Tanuma (2001) argued that, in an environment of high magnetic Reynolds number, like the solar corona and else where in astrophysics, magnetic reconnection must take place in a fractal CS, which is produced by the tearing mode instability. Recent observations from SDO/AIA have also shown clear evidence for turbulence in supra-arcade plasma sheets (Reeves and Golub 2011; McKenzie 2013; Doschek et al. 2014). In such a CS, island-like magnetic structures of various scales relate 
the large scale features of the CS itself and those out of the CS to the micro-scale structures inside the CS in a self-consistent way.

For a CS with large aspect ratio, say larger than $2 \pi$, the tearing mode is linearly unstable (e.g., see Furth et al. 1963; Priest and Forbes 2000). In this stage, the large CS fragments into many structures (namely islands) of intermediate sizes. Continuous evolution in the system leads to the non-linear development of the instability. Smaller islands form in between islands that appeared earlier and are moving apart, which manifests the scenario of filamentation of the CS and has also been discussed by Shibata and Tanuma (2001) and Loureiro et al. (2007). [The instability with flow was considered by Loureiro et al. 2007 as well.] This process yields the successive decrease in the size of magnetic islands until the size of islands approaches the kinetic size of the system so that fast dissipation or fast reconnection is allowed to take place. In this process, the magnetic energy in large scale structures continuously cascades to small scales at which the magnetic field is quickly diffused.

Shibata and Tanuma (2001) found that, in the typical coronal environment, fast reconnection may occur as the number of cascading steps reaches 6 . They noticed that the tearing mode develops slowly in the linear stage, it takes around $3 \times 10^{4} \sim 10^{6} \mathrm{~s}$ for the first island to form after the instability commences. The system then evolves non-linearly, and the formation of magnetic islands accelerates such that the second island appears 100 1000 s after the first one, and the CS quickly fragments. On the other hand, recent numerical experiments indicated that these times depend on the background of the system and the initial configuration of the reconnecting magnetic field. Shen et al. (2011) used an initial configuration commonly applied to model two-ribbon flares with the magnetic field line-tied to the lower boundary and with the other boundaries open. They found that the first island appears $1.6 \times 10^{3} \mathrm{~s}$ after the system starts to evolve as a result of perturbation equivalent to $0.03 V_{A}$ at the open boundary. This time shortens to $900 \mathrm{~s}$ if the perturbation is enhanced to $0.1 V_{A}$ in a similar initial configuration (L. Ni 2014, private communication).

In the simulation of an eruption invoked by the catastrophic loss of equilibrium that produces both CME and flare, the first blob appeared $290 \mathrm{~s}$ after the disruption took place in the relevant magnetic configuration, the second one was created $79 \mathrm{~s}$ after the first one and the third one appeared $21 \mathrm{~s}$ following the second (Mei et al. 2012). In these and other similar numerical simulations (e.g., see Skender and Lapenta 2010; Bárta et al. 2011a; and references therein), the reconnection process always accelerates after the formation of the first plasmoid, which manifests the non-linear property of the tearing mode in the later stage and provides strong support of the result of the analytic studies by Shibata and Tanuma (2001). The non-linear stage of the tearing mode is also known as the plasmoid instability (e.g. see also Bhattacharjee et al. 2009; Huang et al. 2010; Huang and Bhattacharjee 2010; Shen et al. 2011; Murphy et al. 2013; Shen et al. 2013 and references therein).

Forbes and Priest (1982) started working on the magnetic reconnection process taking place in the CS of two-ribbon flares, confirmed the kinematic features of post-flare loops predicted by the Kopp-Pneuman model (Kopp and Pneuman 1976), and investigated the rising of the post-flare loop and the interaction between the sunward reconnection outflow and the flare loop top. They noticed the occurrence of the slow mode shock extending downward from the principal reconnecting region in the CS (see also recent works by Shen et al. 2011; Mei et al. 2012) and the special upside-down Y-shape structure of the two-ribbon flare, and that it took a while for the fast reconnection to commence in the CS.

Forbes and Priest (1983) further studied the non-linear evolution of the reconnecting magnetic configuration. Two new features, which had not been considered by the existing models of the flare loop, appeared in their numerical experiments. These two features were the fast mode shock located between the top of flare loops and a pair of slow mode shocks 
extending from the lower tip of the CS, and the formation and the annihilation of magnetic islands in the CS above the flare loop (see also discussions of Bárta et al. 2011a; Shen et al. 2011; Mei et al. 2012). In the process of magnetic reconnection, some islands move upward and some downward, and they pointed out that the annihilation resulted from the coalescence instability.

We now know that the first phenomenon noticed by Forbes and Priest (1983) is the socalled termination shock on the top of flare loops, and that it results from the interaction of the reconnection outflow with flare loops, namely the product by the high speed flow when meeting an obstacle. It is the same in both the origin and physical properties as the bow shock on the top of the magnetosphere (see discussions of Priest and Forbes 2000, pp. 322-324; Lin et al. 2008), and as the termination shock near the edge of the heliosphere. In the reconnection process, the speed of the outflow is roughly the local Alfvén speed near the principal X-point (e.g., see detailed discussions of Lin et al. 2008; Shen et al. 2011), at which the reconnection rate reaches its maximum. On the other hand, the sunward outflow is usually slowed down by the closed flare loops (see Table 2 of Shen et al. 2011), which could result in the termination shock, and the stationary type II radio burst of nearly zero frequency drift reported by Aurass et al. (2002) constituted a possible evidence of the termination shock. Forbes and Malherbe (1986) further studied the properties of the termination shock, and realized that the termination shock may cause the accumulation of the thermal energy on the flare loop top, enhancing the formation of flare loops below the CS. On the basis of the works by Forbes and Priest (1983) and Forbes and Malherbe (1986), Yokoyama and Shibata $(1997,2001)$ investigated the impact of anisotropic heat conduction and chromospheric evaporation on magnetic reconnection and the consequent formation of flare loops, revealing much information that is more realistic and enriching our knowledge about the relevant issues.

The second feature noticed by Forbes and Priest (1983) has continuously been displayed and duplicated in recent numerical experiments with higher and higher resolutions (Riley et al. 2007; Bárta et al. 2008, 2010, 2011a, 2011b; Shen et al. 2011, 2013; Murphy et al. 2013). These experiments also confirmed that it is inevitable for magnetic islands to form in the CS developed in the coronal environment of high $S$, and that small islands have a tendency to merge into bigger islands. Theoretical and numerical studies by Karlický and Bárta (2011) further indicated that collisions or interactions between two adjacent islands may produce energetic particles (see also Nishizuka and Shibata 2013), and contribute to the HXR and the radio emissions as well.

Subsequent works turned to pay more attention to the small scale structures inside the reconnecting CS as well as the possible observational consequences. In the spirit of Ko et al. (2003) and Lin et al. (2005) regarding the motion of plasma blobs moving in the CS observed in white-light, Riley et al. (2007) simulated evolutionary features of the CS and kinematic behaviors of magnetic islands in the CME/flare CS for the first time, specifying that the motion of the plasma blobs bifurcates somewhere in the CS, and that one group of blobs moved sunward and another one moved anti-sunward. They noticed that the location where the bifurcation took place was not far from the solar surface, and was usually within one solar radius from the solar surface. All the blobs, together with the associated reconnection outflows, observed above this location move anti-sunward, and those below this location move sunward (see also the movies attached to the papers by Ko et al. 2003; Lin et al. 2005; Savage et al. 2010). This explained why the plasma blobs appearing in FOVs of the LASCO C2 and C3 were always observed to leave the Sun, and why those appearing at lower altitudes were always observed to move sunward (e.g., see the events and the associated references listed in Table 2 of Shen et al. 2011). 
In addition to the above behaviors, the results of Shen et al. (2011) revealed that the occurrence of the tearing mode, and thus the formation of magnetic islands, depends on the value of $S$ : magnetic reconnection undergoes smoothly and the CS is dissipated gradually without any small structure appearing in the CS at $S<900$, and the situation changes apparently with the formation of magnetic islands at $S>1000$. As a follow-up of Forbes and Malherbe (1986), Shen et al. (2011) re-investigated the reconnection process taking place in the two-ribbon flare CS in the same magnetic configuration, even using the same code, but with much higher grid resolution and even adaptive mesh refinement (AMR) because of the significant improvement in calculation techniques and resources. So not only did Shen et al. (2011) duplicate all the phenomena observed by Forbes and Malherbe (1986), but they obtained much richer information than before on the reconnection process in the flare CS.

First of all, evolution in the system with asymmetric boundary conditions (namely, the line-tied boundary condition on the bottom side and the open boundary on the other three sides) does not immediately cause the tearing mode to occur although the aspect ratio of the CS exceeds $2 \pi$ greatly at the very beginning, which is the criterion deduced from the linear theory of the tearing mode (e.g., see also Furth et al. 1963; Priest and Forbes 2000). This is consistent with the result of the simulations with symmetric boundary conditions (e.g., see Loureiro et al. 2007; Ni et al. 2010; Mei et al. 2012). Two reasons lead to the difference between analytic solutions and numerical experiments: One, it turned out that the most significant consequence of the tearing mode will not appear until non-linear effects become dominant; and two, comparing with the magnetic configuration studied by Furth et al. (1963), those studied in the numerical experiment contain a reasonably large amount of mass, and could be as close to realistic as possible.

In these numerical experiments, the system commences to evolve as the two magnetic fields of opposite polarity and plasmas at either side of the CS approach one another, most of mass in the CS is expelled, and the CS gets thinner and thinner yielding a larger and larger aspect ratio. Loureiro et al. (2007) found that the first magnetic island, thus the tearing mode instability, occurs after the aspect ratio exceeds 50, and $\mathrm{Ni}$ et al. (2010) noticed that the threshold value of this ratio could be even larger than 100. The threshold value of the ratio in the case of Shen et al. (2011) was not available since they investigated the KoppPneuman configuration (e.g., see Kopp and Pneuman 1976) in which the CS extended from the solar surface to infinity. However, their results clearly showed that the tearing mode did not appear at the very beginning although the aspect ratio of the CS was infinity until the CS was squeezed to be thin enough. Figure 20 displayed space distributions of plasma density $\rho$, electric current density $J, x$-component of velocity $v_{x}$, and $z$-component of velocity $v_{z}$, respectively, at two times of the system in evolution. As expected, many blobs followed the first one to appear in the CS successively with the tearing mode commencing to take place, and then the CS thickness gradually approached a constant (see solid curve in Fig. 21 with $L_{0}$ being the scale of the simulation domain).

Second, the electric current density in the CS gradually increases before the appearance of the first plasma blob, and a significant increment took place following the formation of the first blob. After it reached maximum, the current density roughly stayed constant for a while until a large amount of magnetic field and the associated energy had been dissipated (see dashed curve in Fig. 21 with $J_{0}$ being the characteristic value of the electric current density).

The third important result of Shen et al. (2011) is that a special X-point exists inside the CS as indicated by observations in the CME/flare CS and in the magnetotail CS (see discussions of Lin et al. 2008 and references therein). The reconnection rate, $M_{A}$, is higher at this special X-point than at the other X-points inside the CS, and the sunward and antisunward reconnection outflows bifurcate around this point. This special X-point is known 

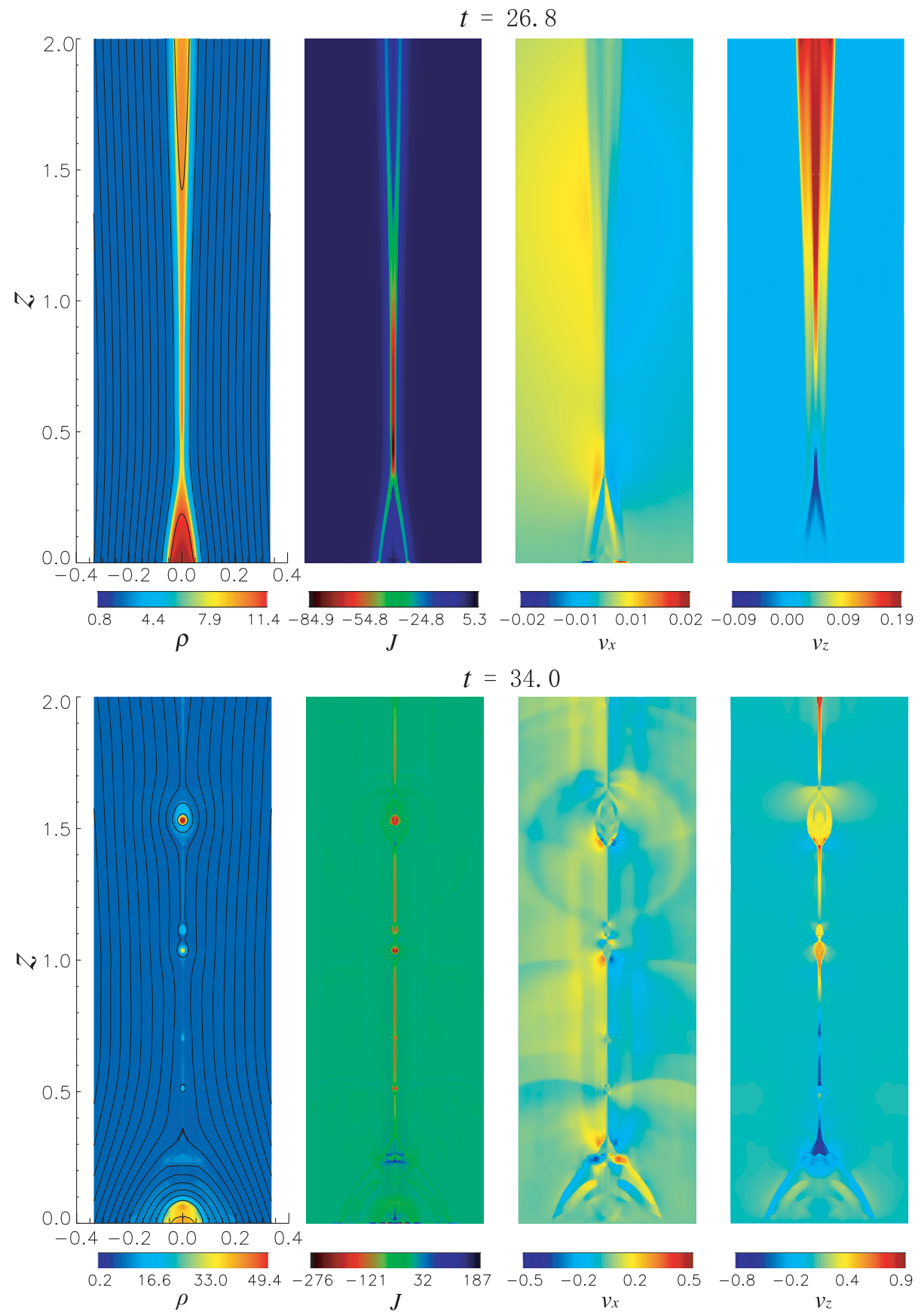

$$
t=34.0
$$

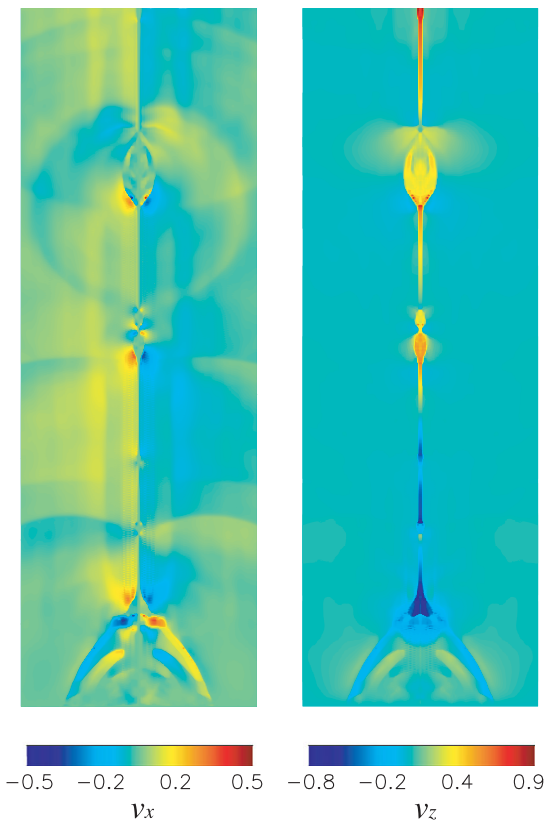

Fig. 20 Evolution of various parameters (plasma density $\rho$, electric current density $J, x$ - and $z$-components of velocity) in the magnetic configuration including a semi-infinitely long CS as magnetic reconnection progresses. Upper row: reconnection is in the Sweet-Parker stage; lower row: reconnection is in the turbulent stage. From Shen et al. (2011) 
Fig. 21 Variations of the half-width $w$ (solid curve) and the electric current density $J$ (dotted curve) of the CS near the PX-point versus time. The arrow specifies the time $t=26.8$ when the first magnetic island (O-point) starts to form. Here $L_{0}$ is the length scale of the simulation domain, and $J_{0}$ is the characteristic value of the electric current density. From Shen et al. (2011)

Fig. 22 Rate of magnetic reconnection $M_{A}$ near the PX-point as a function of time. The solid line shows the instant value and the dotted line is for the corresponding average value. The arrow indicates time $t=26.8$ when the first magnetic island forms. From Shen et al. (2011)
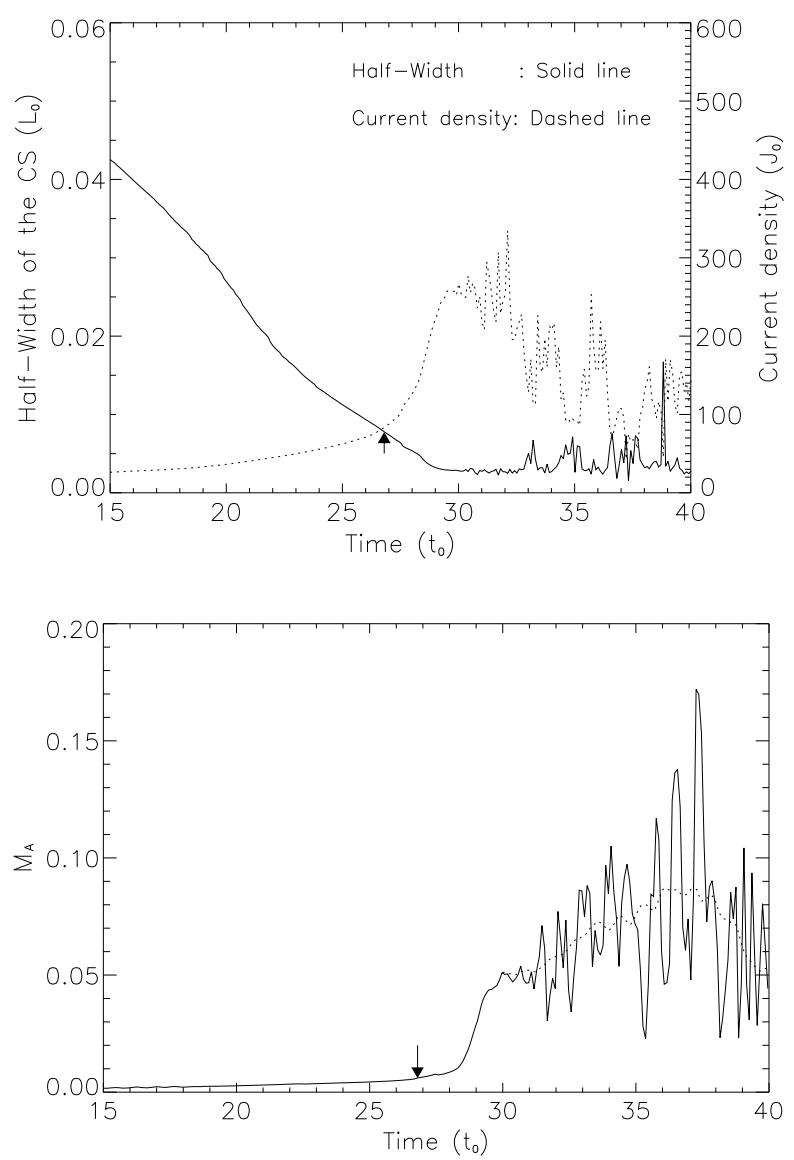

as the principal X-point (or PX-point). As shown by Fig. 20, at an earlier time, $t=26.8$, the reconnection process is at the Sweet-Parker stage, we see a narrowest location at the CS, where the plasma flows bifurcate (see the right panel in the first row); at a later time, $t=$ 34.0, the process evolves to the turbulent stage, many magnetic islands (blobs) associated with many X-points appear, and we see again that motions of this blobs, together with the reconnection outflow, bifurcate at a special point (see the right panel in the second row). This figure shows how the PX-point looks and how it distinguishes from the other X-points. Interested readers are referred to Fig. 6 of Shen et al. (2011), which clearly demonstrates the time-variations of the heights of different X-points. Further studies of the value of $M_{A}$ at the PX-point showed that $M_{A}$ remained small but kept increasing gradually after the first plasma blob was produced in the CS. The consequent evolution in $M_{A}$ manifested an apparent growth and the oscillating behavior, with the highest value close to 0.1 (see the dashed curve in Fig. 22) following the formation of a group of magnetic islands and the associated X-points.

Furthermore, Shen et al. (2011) noticed that a fluid stagnation point (S-point), at which the fluid speed was roughly zero, existed near the PX-point. They were very close to one another, but not co-located with one another in space. They were moving anti-sunward slowly and their relative positions switch back and forth as reconnection progresses. Looking into detailed features of plasma blobs and these two special points reveals that the direction in 
which a plasma blob moves is related to the relative positions of the S- and PX-points. When the S-point is above the PX-point, newly formed blobs move upward. When the S-point is below the PX-point, new blobs move downward. No blob was observed to appear between the S-point and PX-point, this implies that plasma blobs never go through either of the two points.

Plasmoids are not limited to the CS between the CME and the flare arcade. Studies involving the breakout reconnection configuration have shown that X-and O-type nulls and associated plasmoids also appear in the CS that forms ahead of the eruption, referred to as the breakout current sheet. Karpen et al. (2012) found that the shape of the X-points early in the reconnection is very flat and sheet-like, and that they change shape to X-points with broader opening angles soon after the appearance of reconnection jets in the breakout sheet. Such a change in shape indicates a transition from a form of slow tearing in the CS to a faster and strongly driven reconnection process. This metamorphosis in null-point shape is mirrored in the X-point nulls that appear in the flare CS later in the eruption, indicating a similar evolution in the reconnection there. Karpen et al. (2012) also noticed that the number of X-and O-type nulls in the breakout CS are strongly but not perfectly correlated with the number of nulls in the flare CS during the explosive eruption phase, indicating that there is feedback between the two locations where reconnection is occurring.

Recently, Guo et al. (2013) investigated plasmoid instability and its consequences by comparing the distribution of plasmoids obtained from LASCO data in a CME event by Ko et al. (2003) with those deduced from a resistive MHD numerical experiment. They found that the plasmoid scale distribution of their experiment as well as the theoretical and numerical results of Huang and Bhattacharjee (2012) were consistent with observations of Ko et al. (2003). In addition, based on the nonlinear scaling law of the plasmoid instability, they inferred a lower bound on the CS width, assuming the underlying mechanism of CS broadening is resistive diffusion. They noticed that the lower limit to the CS width deduced from the non-linear scaling law was around $3 \times 10^{3} \mathrm{~km}$, which is apparently lower than those obtained by Lin et al. $(2007,2009)$. The difference was ascribed to the fact that Lin et al. $(2007,2009)$ used the linear scaling law and they used non-linear scaling law that seemed more appropriate for studying a turbulent CS (see also Huang and Bhattacharjee 2010).

From an alternative point of view, Bárta et al. (2008) performed a simple statistical analysis for the existing observational results, and found the hypothesis of Kliem et al. (2000) that internal features could be visualized in the radio spectra supported by observations. By using a 2.5-D MHD model, they simulated reconnection in a Kopp-Pneuman configuration with an inhomogeneous and gravitationally stratified atmosphere, and demonstrated the formation and subsequent ejection of plasmoids inside the CS. In their experiments, they tracked the magnetic islands created by the tearing mode as a trap of energetic particles, and calculated the relevant parameters of these islands. According to the results of the experiments, they duplicated the dynamic spectra of the plasma emission from magnetic islands that were consistent with observations.

In several radio bands, on the other hand, observations indicated the occurrence of very fast pulses, but their synthetic dynamic spectra did not display these features. This is probably because the successive production of magnetic islands of various sizes could occur naturally in reality, but small scale structures may not be able to appear in the numerical experiments due to the numerical diffusion as a result of the finite size of the grid. Another reason that causes the difference could be the fact that the plasma beta is large, say $\beta=0.1$, in numerical experiments compared to that in the realistic coronal environment, say $\beta \leq 10^{-3}$. 
Karlický and Bárta (2011) further investigated the production and the coalescence of plasma blobs inside the CS. They found that the free electrons could be apparently accelerated by the coalescence of blobs to very high energy that is enough to produce HXR emission on the top of flare loops. In addition, they noticed that a small CS was created and then got fragmented as two plasma blobs coalesced into one another in the large scale flare CS (see also Nishizuka and Shibata 2013), and particles accelerated in the small CS might be responsible for narrow band decimetric spikes. Further studies and experiments were also performed for the observational consequences of the above process seen in $\mathrm{H} \alpha$, and good agreement was obtained (e.g., see Bárta et al. 2011a).

On the basis of their previous works (Bárta et al. 2008), Bárta et al. (2011b) enhanced the resolution of grids in their experiments, and studied in detail how the magnetic field could be quickly dissipated in the reconnection process. Their results support the theory of Shibata and Tanuma (2001), indicating that the energy associated with large scale structures was successively transferred to that associated with small structures until the kinetic process dominates the energy conversion. This process is similar to that occurring in an incompressible fluid, like water, in which the vortices are created so that the large scale kinetic energy of the fluid flow could be quickly dissipated. Bárta et al. (2011b) found that the coalescence of magnetic islands was as important as the tearing mode instability itself. This is because of the further dissipation of magnetic field by reconnection as plasma blobs coalesce in the CS (see also experiments of Zhong et al. 2010 and the consequences). As the two processes balanced each other, they noticed that, a cascading process, which is suggested by the power-law distribution of the energy versus blob sizes, was eventually set up (Fig. 23). This confirmed both qualitatively and quantitatively the turbulent property of the magnetic reconnection process in the CS, in which large scale structures fill with small structures (e.g., see also detailed discussions of Bemporad 2008).

Statistical descriptions for magnetic islands formed in CS have recently been developed. Using the flux contained in the magnetic island and the area it encloses as two parameters, Fermo et al. $(2010,2012)$ studied the distribution functions of the islands. Their results indicated that the distribution function was determined by the generation of secondary islands, the growth rate of islands, and their merging (or coalescence). The exponential tail of the distribution function as a function of flux was found in their numerical experiments. Numerical simulations of Loureiro et al. (2012) confirmed the inverse square law found by Uzdensky et al. (2010) between the self-similar distribution function of plasmoid size and fluxes. Loureiro et al. (2012) specified that the plasmoid flux and half-width distribution functions scale as an inverse square law.

Later, Shen et al. (2013) performed a set of two dimensional resistive MHD simulations to study the statistical properties of the fine structure and the dependence of the spectral energy on these properties in the two-ribbon flare CS on the basis of their previous work (Shen et al. 2011). They found that the flux and size distribution functions of plasmoids roughly follow inverse square power laws at large scales (Fig. 24a), that the mass distribution function was steep at large scales and shallow at small scales (Fig. 24b), and that the size distribution also showed that plasmoids were highly asymmetric soon after being formed, while older plasmoids tended to be more circular (Fig. 24c). Their results indicated that spectral profiles of magnetic and kinetic energy inside the current sheet were both consistent with a power law. The corresponding spectral indices were found to vary with the Lundquist number $S$ of the system, but tended to approach a constant for large $S\left(>10^{5}\right)$. It may be worth noting that SADs also manifested round and elongated geometric characteristics (e.g., see Hanneman and Reeves 2014) although plasmoids and SADs are different from one another. 
Fig. 23 One-dimensional scale analysis of magnetic field structure along the line $x=0$. (a) Magnetic field lines and current-density structure (green) in the computational domain at $t=316$. The $z$-axis shows positions both in units of LA (top) and in kilometers according to the scaling adopted in Sect. 2. (b) Profile of the $B_{x}$ component of magnetic field along the line $x=0$. (c) Fourier power spectrum of the $B_{x}$ profile.

(d) Wavelet power spectrum of the $B_{x}$ profile. From Bárta et al. (2011b)
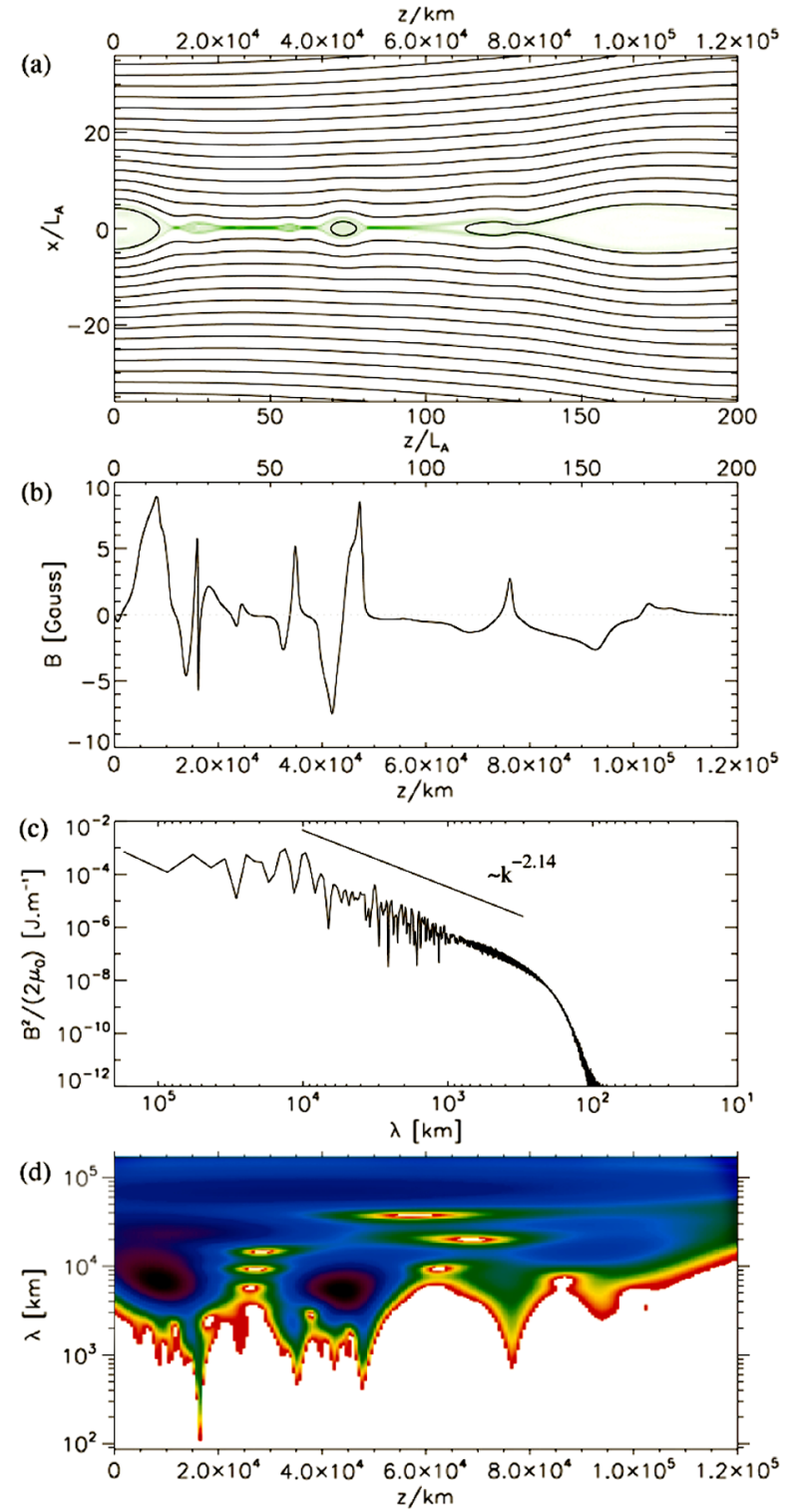

The motion and growth of blobs changed the spectral index such that the growth of new islands and the coalescence of islands caused the power spectrum to steepen, but it became shallower when old and large plasmoids left the computational domain. A very important result obtained by Shen et al. (2013) is that cascading not only happens to the magnetic energy, but happens to the kinetic energy of the plasma flow in the CS as well, which implies a double-diffusive process in which dissipations in both magnetic field and plasma flow take place simultaneously, and the reconnection process occurring in such an environment would diffuse the magnetic field and convert the associated energy into the other types of energy in a significantly efficient way. 

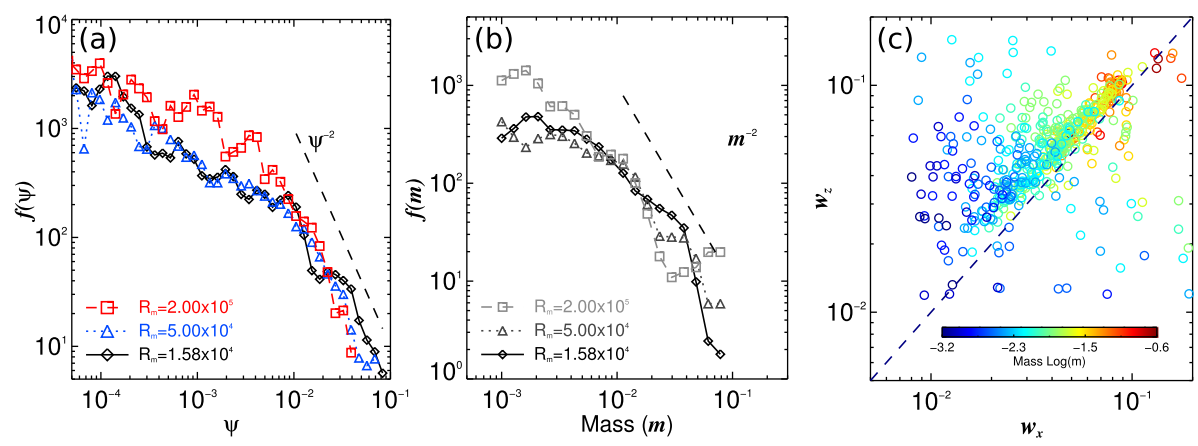

Fig. 24 (a) Plasmoid flux distribution function $f(\Psi)$ for different $R_{m}$. The dashed straight line represents $f(\Psi) \sim \Psi^{-2}$. (b) Plasmoid mass distribution function $f(m)$ for different $R_{m}$. Here, $m$ is the amount mass contained inside each plasmoid. The dashed straight line shows an $m^{-2}$ power law for reference. (c) Plasmoid width distribution in two directions for the case of $R_{m}=5 \times 10^{4}$. Here, $w_{z}$ and $w_{x}$ are the widths of each plasmoid along the $z$ - and $x$-directions, respectively. Color-bar indicates the mass contained in each plasmoid. From Shen et al. (2013)

Similar motion patterns and behaviors of plasmoids inside the flare CS have also reported previously by Forbes and Priest (1982), Riley et al. (2007), Bárta et al. (2011a, 2011b), and so on, cascading of the magnetic energy was also found by Bárta et al. (2011a, 2011b). Meanwhile, Ni et al. (2012b, 2013) performed similar analyses for the magnetic reconnection process in the Harris sheet with $R_{m}$ up to $10^{6}$, and noticed the same cascading feature of the kinetic energy as well. Hence, cascading in the kinetic energy should be a natural and common phenomenon of magnetic reconnection that takes place in the environment of large $R_{m}$.

In addition to the complex pattern that consists of plasma blobs and secondary turbulence due to the blob interactions (Fig. 25a), Mei et al. (2012) noticed the formation of the Petschek slow mode shocks that were located near the edge of the CS. Looking into the plasma flow and magnetic structures around the CS, they recognized the characteristics of Petschek-type reconnection, together with the plasma blob and the consequence of the interaction among blobs (Fig. 25b). The plasma flow pattern and the magnetic field in that region manifested clearly the slow-mode shock of the Petschek type. This was, however, different from the result of the standard theory of the Petschek reconnection, which predicted the angle spanning between two slow shocks of about $7^{\circ}$, the angle measured in their experiments ranged from $6 .{ }^{\circ} 5$ to $14 .^{\circ} 1$. They ascribed this further expansion of the region between the slow shocks to the turbulence of the plasma in the region. Therefore, broadening of the reconnecting CS could be due to the combined effect of the turbulence and the development of the slow-mode shock (see also discussions of Lin et al. 2008, 2009).

No matter whether the detailed physical properties of the Petschek-type reconnection have been fully understood, the work of Mei et al. (2012) revealed that a CME/flare CS possesses much more complex internal structures than expected. The role of these structures are two-fold. First, they are small compared to the whole CS, which apparently enhances the diffusion in the CS; and second, they provide extra pressure to balance that of the reconnection inflow, allowing the CS to have a finite thickness. Therefore, the rate of magnetic reconnection could remain high even in a thick CS, and a reasonably high rate of energy conversion required for the major eruption could be guaranteed. More important, Mei et al. (2012) found that the reconnection outflow between a pair of Petschek shocks is faster than that near the shock, therefore, the magnetic field inside the CS manifests a W- or M-shape 


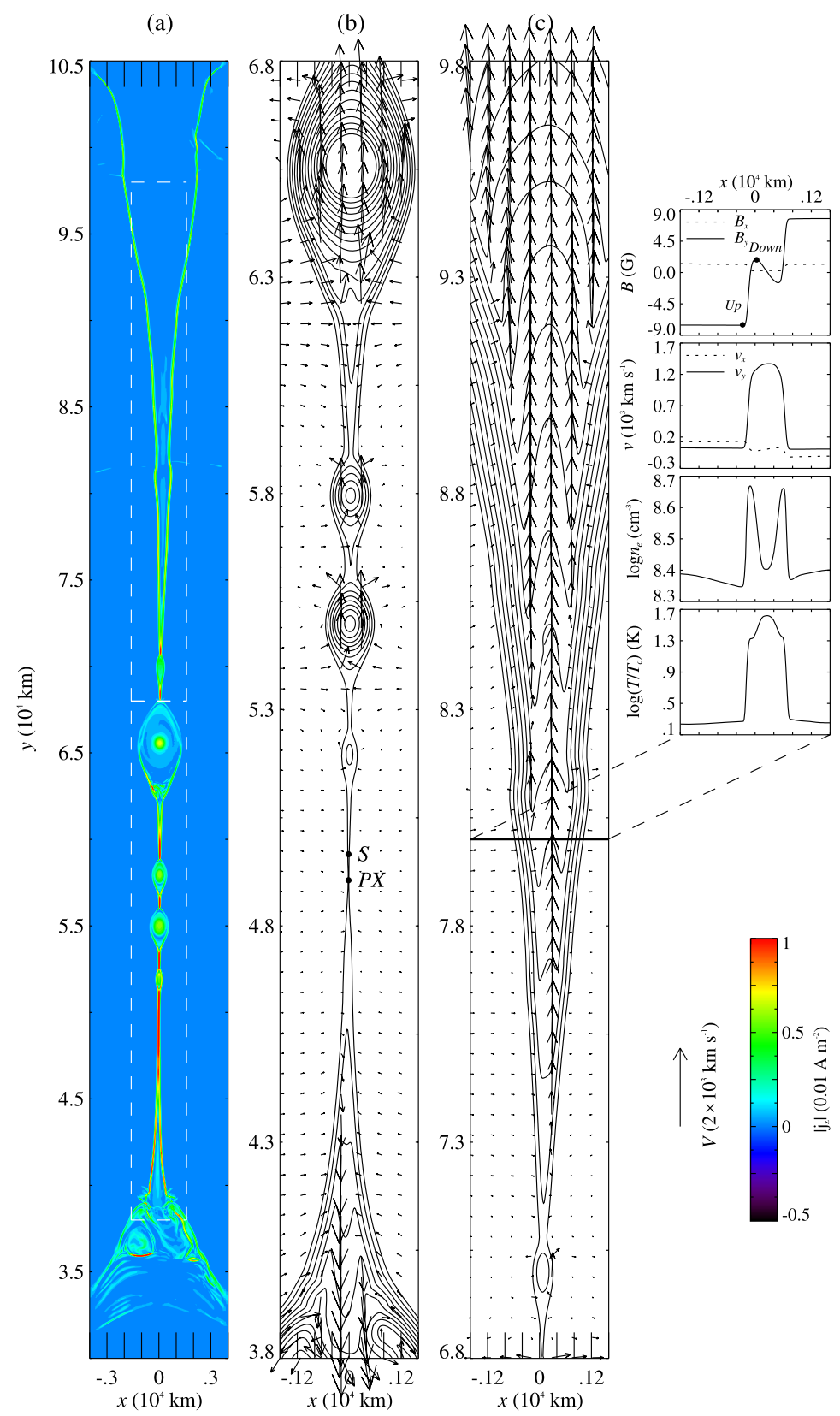

Fig. 25 Detailed features of various parameters around the CS seen at $t=29 \mathrm{~min}$ in the experiment of Mei et al. (2012). Distribution of the electric current is shown in (a), and two smaller rectangular regions surrounded by dashed lines are specified for studying the other parameters in more details. The lower region (b) includes several magnetic islands, the post-flare loop system, as well as the S- and PX-points, which manifests a scenario of the turbulent reconnection; the upper region (c) displays two slow-mode shocks at either side of the outflow region, and shows typical features of the Petschek-type reconnection. The distributions of parameters along a cut marked on (c) are shown by an inset at the upper right-hand corner. From Mei et al. (2012) 
configuration (Fig. 25c) and the magnetic field near the CS center does not monotonically vanish, instead it shows a small overshoot with its amplitude compared to that in the inflow region being roughly $M_{A}$ (T.G. Forbes 2012, private communications). The W-shape magnetic field inside a CS was predicted several decades ago by Vasyliunas (1975) in theory, but had never been confirmed by the numerical experiment before Mei et al. (2012).

All of these facts suggest that the CS is a complex assembly that includes magnetic islands (see also Drake et al. 2006; Lazarian et al. 2012), turbulent flows and termination shocks produced as islands collide with one another and with the closed magnetic structures near two tips of the large CS (e.g., see also Mei et al. 2012; Ni et al. 2012b; and references therein), respectively, and even the slow mode shocks of the Petschek-type in some segments of the CS (e.g., see also theoretical discussions of Vasyliunas 1975; and experimental results of Mei et al. 2012). These macro and micro structures could give rise to important observational consequences manifesting in various aspects of either the CS itself (e.g., see discussions of Bemporad 2008; Lin et al. 2007, 2009; Vršnak et al. 2009) or the output of the reconnection process, namely rapid energy release that results in the intensive heating and acceleration of the plasma as well as production of energetic particles (e.g., see Martens and Young 1990; Litvinenko 1996; Li and Lin 2009; Shibata and Magara 2011; Zharkova et al. 2011; Li et al. 2013b; Nishizuka and Shibata 2013; and references therein).

Before ending this part of work, we need to note here that we are mainly focusing on $2 \mathrm{D}$ and $2.5 \mathrm{D}$ models dealing with the formation of the plasmoid in the CS, and we did not discuss the full 3D scenario in detail. This is because the three-dimensional nature of the tearing mode and related plasmoid instability is not well understood in the context of the solar atmosphere although several groups have made some progress in developing fully 3D MHD models. Analytically, Baalrud et al. (2012) used reduced MHD to investigate the oblique nature of the 3D plasmoid instability. Beresnyak (2013) presents 3D MHD resistive simulations in which turbulence driven by reconnection inhibits the development of plasmoids and flux ropes. Wyper and Pontin (2014a, 2014b) present simulations of null point reconnection that show the formation of flux ropes which are the 3D analogs of 2D magnetic islands. Fully kinetic 3D simulations by Daughton et al. (2011) show that the current sheet develops into a tangled web of interconnected flux ropes, which is likely to also occur in 3D resistive MHD simulations. Over all, developing 3D model is the inevitable tendency, it is worth investing more effort in this issue in the future.

\section{Summary}

We briefly reviewed and looked back on studies of the CS occurring in solar eruptive events in both theory and observations, especially those performed after the catastrophe model for the solar eruption was developed and the CS was identified for the first time in observations. [We may need to point out here as well that observations of Aurass et al. (2013) and Aurass (2014) showed the occurrence of magnetic reconnection both at the top of the disrupting magnetic configuration and above the post-flare loops. This suggests that the large-scale CS could also develop in the fashion of the break-out model. But these observations are very difficult, and only possible if the eruptive process is near but not at the limb.] Further studies through observations of the CS region revealed that the CME/flare CS could be as thick as a few times $10^{4} \mathrm{~km}$, even up to $10^{5} \mathrm{~km}$ (Lin et al. 2007)! This result was not expected previously from the traditional theory of magnetic reconnection and the current sheet. We discussed the question what modifications are needed in the theory and in data interpretation to consider the observed broad tail behind CMEs as a signature of the predicted long CS. 
Traditionally, it was believed that the thickness of the CS should be as small as the skin depth of the plasma, which is about several meters, not bigger than $1 \mathrm{~km}$, in the coronal environment because the energy conversion or the magnetic field dissipation occurring inside the CS must be efficient enough to support the fast energy release in a typical solar eruption that produces both CME and solar flare. To our surprise, the measurement of the CS thickness, $d$, by Lin et al. (2007) indicated that the value of $d$ could be up to several tens of thousand $\mathrm{km}$, which is significantly larger than what was previously expected. More interesting and more important, one always ended up with the similar results for $d$ for different events no matter in which way the value of $d$ was deduced, and no matter in which wavelength the CS was observed as long as it could be recognized (e.g., see Ciaravella et al. 2013; Ling et al. 2014; and references therein). We do not know yet whether this "agreement" could reveal physically valuable information, we shall keep an eye on this issue, and continue to look into it in more detail from different viewpoints in the future. Studies of the projection effects indicated that the impact of the projection effects on the direct measurement of $d$ could be limited, and might not be able to result in an apparent value of $d$ that is more than, at least, 4 orders of magnitude larger than the true value. This is suggestive of that an observed thick CS may not be due to projection effects and complex structures of the $\mathrm{CS}$, but due to the intrinsic physical properties of the reconnection process.

Both observational features of a CS and the related theoretical investigations indicated that the reconnection process is governed by the turbulence and the resultant small structures (also known as the fractal CS) inside the CS. So the thickness of the CS and the rate of reconnection could not be determined by dynamic behaviors and properties of individual particles, but should be controlled by the tearing mode, together with the resultant plasmoid instability. The magnetic energy cascades from large scale turbulent eddies (or magnetic islands) to smaller ones until the diffusion becomes significant. In addition, vorticities, S-points, and velocity shears were also seen in the reconnection outflow (McKenzie 2013), and the secondary instabilities of the Rayleigh-Taylor type has also been used to explain observational features of the CS (Innes et al. 2014).

On the other hand, collisions, or coalescences, among magnetic islands and/or between magnetic islands and the other kind of magnetic structures nearby take place as well (see Mei et al. 2012), which constitutes an inverse cascading process, in the CS. This is not a simple merging of two fluid elements, but accompanied with reconnection between the magnetic fields within different islands, which results in the secondary dissipation of the magnetic field inside the CS. A dynamical balance of cascading and coalescence is usually reached in the reconnection process (e.g., see Bárta et al. 2011a, 2011b). Analyses by Shen et al. (2013) also revealed that the similar cascading behavior occurred to the kinetic energy of the plasma in the CS simultaneously, which implies that the dissipation exists in the fluid motion as well. The laser-driven magnetic reconnection experiments could be considered as possible confirmation of plasmoid collisions and consequences (Zhong et al. 2010) that take place in the flare CS. Furthermore, Mei et al. (2012) found that a CME/flare CS is in fact a complex assembly that includes not only small structures of various sizes but also even the slow mode shocks! We therefore reach the conclusion that the occurrence of various diffusive structures inside the CS allows the reconnection process to take place at a reasonably fast rate even if the CS is thick.

Therefore, it is not easy and straightforward, for the time being, to predict what specific type of reconnection and associated structures of the reconnection would occur in a specific event, or even at a specific stage of an event. The reconnection process could be of either Sweet-Parker, or Petschek, or turbulent, or the combination of these fashions. More effort needs to be invested in looking into detailed processes of magnetic reconnection and the 
physical properties of the CS in the future, and we have identified the region in which the magnetic diffusion is taking place or has taken place with the CS in a more general sense in our previous and the present works.

The above results improved our knowledge about the internal structure and physical properties of the CME/flare CS in an obvious way, and have apparent significance to works in the other fields. First of all, a long and thick CS is favorable for charged particle acceleration. Currently, the scale of the CS is small, of order a few meters thick and tens of meters long, in existing models of the particle acceleration in the CS (e.g., see Litvinenko 1996; Wood and Neukirch 2005; Dauphin et al. 2007). Particles cannot remain long in such a CS, say $\sim 10^{-5}$ s for electrons and $<10^{-3}$ s for protons, so the efficiency of acceleration is fairly low and it is difficult to fulfill the requirement for the rate of production of energetic electrons (> $10 \mathrm{keV}, 10^{35} \mathrm{~s}^{-1}$ ) in a typical solar flare (Martens 1988). Recent studies by Mann et al. (2009) found that observations of the 2003 October 28 event by RHESSI indicated that up to $10^{36}$ electrons with energies $20 \mathrm{keV}$ need to be produced every second to provide a power of about $10^{22} \mathrm{~W}$ to drive a major flare.

In the case of a thick CS that includes turbulence, on the other hand, both electrons and the other charged particles would have larger space to move and stay in the acceleration region longer than in the case of a thin CS, which could enormously enhance the acceleration efficiency, and the turbulence would modulate the particle motion and result in the spectra that are often observed. Detailed features of the particle acceleration in a CS are still open question, and more effort is needed in further and deeper research (e.g., see Dauphin et al. 2007; Li and Lin 2009, 2010, 2012, 2013a, 2013b). The role of small scale structures due to turbulence of the CS in particle acceleration has recently studied by Nishizuka and Shibata (2013) who found that the interaction of these structures provides an approach to Fermi acceleration for particles. Mann and Warmuth (2011) pointed out that, in a medium class flare, if about $8 \%$ of the all electrons brought into CS by reconnection inflow are accelerated, they are able to bring about $12 \%$ of the total released energy into the flare region; in a major flare, on the other hand, up $60 \%$ total inflow electrons need to be accelerated to the energy $>20 \mathrm{keV}$ to account for $60 \%$ the total released energy observed in the flare region. In both cases, they found that the slow-mode shock plays a role in heating and the termination shock plays a role in particle accelerations.

In addition, our previous understanding of the plasma instabilities and turbulence is on the scale of laboratory or the skin depth of protons, which is usually several meters in the coronal environment (Drake et al. 2006). However, observations of the CME/flare $\mathrm{CS}$ indicate that the similar processes or phenomena could take place on the scale from $10^{4}$ to $10^{5} \mathrm{~km}$, which implies that some theories and understandings on the basis of the lab scale need to be modified and improved. Bemporad (2008) provided observational evidence for such works that have already been started (e.g., see the recent serial of the works by Bárta and co-workers, Shen et al. 2011; Mei et al. 2012; Shen et al. 2013). Meanwhile, the work by Shibata and Tanuma (2001) deserves more attention and some issues discussed by them are also worth further investigation.

The CME/flare CS is a relatively new topic that started drawing our attention about 15 years ago. Research on many important issues have commenced just recently, and our knowledge on the internal structure and the corresponding properties is still fairly preliminary. Among the issues that draws most debate is probably the value of the CS thickness. Although observations continue to confirm the significantly large thickness of the CS, all the results obtained so far were the FWHM of the brightness profile of the CS, not the FWHM of the electric current in the CS. This is because all we can currently observe is the plasma emission, not the electric current sheet. Therefore, there are effects like the thermal halo 
that could create a sheath of plasma around the CS, yielding uncertainties in determining $d$ if the measurement is on the basis of the brightness at a specific wavelength that also depends on the plasma temperature. However, we may need to note here as well that the brightness in white-light depends on the electron density only, and hence the measurement of $d$ on the basis of the white-light data is not affected by the thermal halo. Furthermore, Ciaravella and Raymond (2008) measured $d$ based on the WL data and the UVCS data that were taken simultaneously at the same location. Their results show that the values of $d$ measured from WL and UVCS data are the same, which implies that if a thermal halo exists, the gas in this region is less dense than that inside the CS and may not contribute much to the EM. Hence the thermal halo is unlikely to have a big effect on measurement of $d$.

There is currently no existing technique that allows us to directly measure or detect the distribution of the electric current (density or intensity) inside the CS. So for the time being, studying the brightness distribution is the only approach via which more or less, we are able to look into the geometric structure and physical properties of the CS. In addition to the thermal halo, it is possible that, like the case of the helmet streamer, the CME/flare CS itself is actually very thin and embedded in a thick plasma sheet that could be recognized according to the brightness, and the CS thickness is actually much smaller than that of the plasma sheet (e.g., see also Song et al. 2012).

But in the framework of Lazarian and Vishniac (1999), magnetic reconnection is turbulent, and the reconnection region fills with turbulent eddies, which results in a broad reconnection region, so the plasma sheet and the CS fill the same region in space. Kowal et al. (2009) studied numerically the effects of turbulence on magnetic reconnection using three-dimensional direct numerical simulations, and tested the model of fast magnetic reconnection developed by Lazarian and Vishniac (1999). The numerical simulations duplicated the Sweet-Parker CS in the absence of turbulence. Kowal et al. (2009) noticed that the effect of the Ohmic dissipation and that of the Hall MHD disappeared as turbulence was turned on in the CS. Further investigations of the internal structures of CS were performed by Vishniac et al. (2012), and the fact was noticed that peaks of the electric current were distributed throughout the reconnection region. This confirmed that the CS and the reconnection region (or the plasma sheet) are nearly co-located in space with one another, and are filled with turbulent structures. But their results were not tested for the case of weak turbulence, which could be a weakness of the study (e.g., see also Lazarian et al. 2014).

Overall, the geometric structure and the internal features of the CME/flare CS is still an open question, and the true value of $d$ as well as correct approaches to measuring $d$ constitutes an important topic that needs us to invest more effort in both observational and theoretical studies. Therefore, we note here that the terms "diffusion", "dissipation", and "current sheet" have actually been used in a more general sense in this and our previous works than were used traditionally to refer to any process that causes magnetic diffusion and any region where such diffusion occurs (e.g., see also discussions of Lin and Forbes 2000). In this sense, some parameters, like $\eta_{e}$ and $d$ determined by either high temperatures observed by UVCS or high densities observed by coronagraphs, should be considered effective and average.

Different from our previous concepts that the cascading process transfers the energy associated with large scale structures to that associated with small scales until the kinetic process dominates, recent numerical experiments for magnetic reconnection revealed that an inverse process, namely merging or coalescence of magnetic islands, takes place simultaneously (e.g., see Bárta et al. 2011a, 2011b; Shen et al. 2011, 2013; Mei et al. 2012; Ni et al. 2012a, 2012b, 2015). It is probably this inverse process that causes the energy spectra, 
which is usually a power law, to possess an index steeper than that of the Kolmogorov value of $5 / 3$. The indices of the energy spectra appearing in these experiments usually ranges from 2 to 6 . More attention has been theoretically and numerically drawn to this issue recently, and further and deeper understanding about it also requires high quality and high resolution observations. Great progress could be expected should the small scale features of a CS and the associated properties be recognized and understood in the future.

Here the high quality observations first refer to the direct measurement of the coronal magnetic field with which we are able to perform the spectral analyses about the magnetic energy in the CS as we have done for the corresponding numerical results, second to the 3D measurements of various parameters for the CS so that the kinetic energy of plasma blobs can be obtained and the similar spectral analyses about the kinetic energy could be performed, and third to spending more time and effort in analyzing and studying the existing data in order to acquire important information that might have been missed in previous works; the high resolution observations refers to the observations of the CS with spatial resolution, velocity resolution, and time cadence like those obtained with the Interface Region Imaging Spectrograph (IRIS, De Pontieu et al. 2014), or even better. IRIS provides simultaneous spectra and images of the photosphere, chromosphere, transition region, and corona with $0.33-0.4$ arcsec spatial resolution, $2 \mathrm{~s}$ time cadence, and $1 \mathrm{~km} \mathrm{~s}^{-1}$ velocity resolution over a FOV up to $175 \times 175 \operatorname{arcsec}^{2}$.

Another important remaining topic for future work is the nonlinear evolution of the tearing and plasmoid instabilities in three dimensions. Particle-in-cell (PIC) simulations of small-scale reconnection by Daughton et al. (2011) show that plasmoids and magnetic islands in 2D correspond to highly structured flux ropes in 3D. In the future, it will be important to couple these small-scale, fully kinetic simulations to larger scales where the resistive MHD approximation is more appropriate. The nonlinear evolution of the plasmoid instability in 3D has important implications on the interpretation of blobs observed in current sheets (e.g., Ko et al. 2003; Guo et al. 2013).

Last but not least, we note that the CS in the real eruptive process forms and quickly develops in the early stage of the solar flare, which is the most dynamic phase of the eruption, and magnetic reconnection takes place in a region that itself is during a violently changing process. However, most numerical experiments on reconnection so far known were started with an pre-existing CS that starts in mechanical and thermal equilibrium, and the reconnection process commences after a perturbation to the system is introduced. To our knowledge, Riley et al. (2007) and Mei et al. (2012) are probably so far the known numerical works that paid significant attention to the details of the interior of the CS that was created in a disrupting magnetic configuration. In the work of Reeves et al. (2012), formation of plasma blobs in the CS that was developed dynamically were noticed, but no further investigation on internal structures of the CS was performed. Therefore, our knowledge about magnetic reconnection occurring in a dynamically developing CS must be improved and/or renewed. We need to study in the future the occurrence of the plasma instabilities and the resultant turbulence in a fast evolving CS, to look into their properties that might be different from those appearing in a static CS, and to investigate possible implications of these features to particle acceleration in the CME/flare CS as well.

Acknowledgements JL thanks the organizer of the Hinode-7 Science Meeting for invitation to give an overview on the related topic, and the main content of this paper was reported at the meeting. This work was supported by Program 973 grant 2013CBA01503, NSFC grants 11203069, 11273055, 11333007, and 11403100, CAS grant XDB09040202, Yunnan Province grant 2011FB113, as well as NSF SHINE grants AGS-1156076 and AGS-1358342. 
Open Access This article is distributed under the terms of the Creative Commons Attribution 4.0 International License (http://creativecommons.org/licenses/by/4.0/), which permits unrestricted use, distribution, and reproduction in any medium, provided you give appropriate credit to the original author(s) and the source, provide a link to the Creative Commons license, and indicate if changes were made.

\section{References}

T. Amari, J.F. Luciani, J.J. Aly, Astrophys. J. 629, L37 (2005)

T. Amari, J.J. Aly, Z. Mikić, J. Linker, Astrophys. J. 717, L26 (2010)

J. Ambrosiano, W.H. Matthaeus, M.L. Goldstein, D. Plante, J. Geophys. Res. 93, 14383 (1988)

S.K. Antiochos, Astrophys. J. 502, L181 (1998)

S.K. Antiochos, C.R. DeVore, J.A. Klimchuk, Astrophys. J. 510, 485 (1999)

A. Asai, T. Yokoyama, M. Shimojo, K. Shibata, Astrophys. J. 605, L77 (2004)

M.J. Aschwanden, Astrophys. J. 608, 554 (2004)

H. Aurass, Sol. Phys. 289, 4517 (2014)

H. Aurass, G. Mann, Astrophys. J. 615, 526 (2004)

H. Aurass, B. Vršnak, G. Mann, Astron. Astrophys. 384, 273 (2002)

H. Aurass, G. Mann, G. Rausche, A. Warmuth, Astron. Astrophys. 457, 658 (2006)

H. Aurass, F. Landini, S. Braune, G. Poletto, Astron. Astrophys. 506, 901 (2009)

H. Aurass, G. Mann, P. Zlobec, M. Karlicky, Astrophys. J. 730, 57 (2011)

H. Aurass, G. Holman, S. Braune, G. Mann, P. Zlobec, Astron. Astrophys. 555, A40 (2013)

S.D. Baalrud, A. Bhattacharjee, Y.-M. Huang, Phys. Plasmas 19, 022101 (2012)

H.M. Bain, S. Krucker, L. Glesener, R.P. Lin, Astrophys. J. 750, 44 (2012)

M. Bárta, M. Karliký, R. Žemlička, Sol. Phys. 253, 173 (2008)

M. Bárta, J. Büchner, M. Karliký, Adv. Space Res. 45, 10 (2010)

M. Bárta, J. Büchner, M. Karliký, P. Kotrč, Astrophys. J. 730, 47 (2011a)

M. Bárta, J. Büchner, M. Karliký, J. Skála, Astrophys. J. 737, 24 (2011b)

H. Baty, E.R. Priest, T.G. Forbes, Phys. Plasmas 16, 060701 (2009)

A. Bemporad, Astrophys. J. 689, 572 (2008)

A. Bemporad, G. Poletto, S.T. Suess et al., Astrophys. J. 638, 1110 (2006)

A.R. Beresnyak, arXiv:1301.7424 (2013)

A. Bhattacharjee, Y. Yuan, Astrophys. J. 449, 739 (1995)

A. Bhattacharjee, Y.M. Huang, H. Yang, B. Rogers, Phys. Plasmas 16, 112102 (2009)

L. Biermann, Z. Naturforsch. 5a, 65 (1950)

D. Biskamp, Phys. Fluids 29, 1520 (1986)

D. Biskamp, Nonlinear Magnetohydrodynamics (Cambridge University Press, Cambridge, 1993), p. 22

D. Biskamp, Magnetic Reconnection in Plasmas (Cambridge University Press, Cambridge, 2000), p. 256

N.A. Bobrova, S.I. Syrovatskii, Sov. J. Plasma Phys. 6, 1 (1980)

H. Carmichael, in AAS-NASA Symp. Phys. Solar Flares (1964), p. 451

A. Caspi, R.P. Lin, Astrophys. J. 725, L161 (2010)

P.A. Cassak, M.A. Shay, Space Sci. Rev. 182, 283 (2012)

P.A. Cassak, J.F. Drake, J.T. Gosling, T.D. Phan, M.A. Shay, L.S. Shepherd, Astrophys. J. 775, L14 (2013)

M. Cécere, M. Schneiter, A. Costa, S. Elaskar, S. Maglione, Astrophys. J. 759, 79 (2012)

P. Chen, K. Shibata, D.H. Brooks, H. Isobe, Astrophys. J. 602, L61 (2004)

X. Cheng, J. Zhang, Y. Liu, M.D. Ding, Astrophys. J. 732, L25 (2011)

A. Ciaravella, J.C. Raymond, Astrophys. J. 686, 1372 (2008)

A. Ciaravella, J.C. Raymond, J. Li, P. Reiaer et al., Astrophys. J. 575, 1116 (2002)

A. Ciaravella, D.F. Webb, S. Giordano, J.C. Raymond, Astrophys. J. 766, 65 (2013)

W. Daughton, V. Roytershteyn, H. Karimabadi, L. Yin, B.J. Albright, B. Bergen, K.J. Bowers, Nat. Phys. 7, 539 (2011)

C. Dauphin, N. Vilmer, A. Anastasiadis, Astron. Astrophys. 468, 273 (2007)

J.A. Davies, R.A. Harrison, A.P. Rouillard, N.R. Sheeley Jr., C.H. Perry, D. Bewsher et al., Geophys. Res. Lett. 36, L02102 (2009)

C.J. Davis, J.A. Davies, M. Lockwood, A.P. Rouillard, C.J. Eyles, R.A. Harrison, Geophys. Res. Lett. 36, L08102 (2009)

B. De Pontieu, A.M. Title, J.R. Lemen, G.D. Kushner, D.J. Akin, B. Allard et al., Sol. Phys. 289, 2933 (2014)

P. Démoulin, J.C. Henoux, E.R. Priest, C.H. Mandrini, Astron. Astrophys. 308, 648 (1996)

G.A. Doschek, D.E. McKenzie, H.P. Warren, Astrophys. J. 788, 26 (2014)

J.F. Drake, M. Swisdak, K.M. Schoeffler et al., Geophys. Res. Lett. 33, L13105 (2006) 
J.W. Dungey, Philos. Mag. 44, 725 (1953)

J.P. Eastwood, T.-D. Phan, F.S. Mozer, M.A. Shay, M. Fujimoto, A. Retinó et al., J. Geophys. Res. 112, 6235 (2007)

G. Einaudi, S. Chibbaro, R.B. Dahlburg, M. Velli, Astrophys. J. 547, 1167 (2001)

D.F. Elmore, J.T. Burkepile, J.A. Darnell, A.R. Lecinski, A.L. Stanger, in Polarimetry in Astronomy, ed. by S. Fineschi. Proceedings of the SPIE, vol. 4843 (2003), p. 66

Y. Fan, S.E. Gibson, Astrophys. J. 668, 1232 (2007)

R.L. Fermo, J.F. Drake, M. Swisdak, Phys. Plasmas 17, 010702 (2010)

R.L. Fermo, J.F. Drake, M. Swisdak, Phys. Rev. Lett. 108, 255005 (2012)

T.G. Forbes, Geophys. Astrophys. Fluid Dyn. 62, 15 (1991)

T.G. Forbes, L.W. Acton, Astrophys. J. 459, 330 (1996)

T.G. Forbes, P.A. Isenberg, Astrophys. J. 373, 294 (1991)

T.G. Forbes, J. Lin, J. Atmos. Sol.-Terr. Phys. 62, 1499 (2000)

T.G. Forbes, J.M. Malherbe, Astrophys. J. 302, L67 (1986)

T.G. Forbes, J.M. Malherbe, Sol. Phys. 135, 361 (1991)

T.G. Forbes, E.R. Priest, Sol. Phys. 81, 303 (1982)

T.G. Forbes, E.R. Priest, Sol. Phys. 84, 169 (1983)

T.G. Forbes, E.R. Priest, Rev. Geophys. 25, 1583 (1987)

T.G. Forbes, E.R. Priest, Astrophys. J. 446, 377 (1995)

T.G. Forbes, E.R. Priest, P.A. Isenberg, Sol. Phys. 150, 245 (1994)

T.G. Forbes, J.A. Linker, J. Chen, C. Cid, J. Kota, M.A. Lee et al., Space Sci. Rev. 123, 251 (2006)

H.P. Furth, J. Killeen, M.N. Rosenbluth, Phys. Fluids 6, 459 (1963)

P.T. Gallagher, Sol. Phys. 210, 341 (2002)

G. Gao, M. Wang, J. Lin, N. Wu, C. Tan, B.H. Kliem, Y. Su, Res. Astron. Astrophys. 14, 843 (2014)

L. Glesener, S. Krucker, H.M. Bain, R.P. Lin, Astrophys. J. 779, L29 (2013)

N. Gopalswamy, S. Yashiro, G. Michalek, G. Stenborg, A. Vourlidas, S. Freeland, R. Howard, Earth Moon Planets 104, 295 (2009)

S.E. Guidoni, D.W. Longcope, Astrophys. J. 730, 90 (2011)

L.J. Guo, A. Bhattacharjee, Y.M. Huang, Astrophys. J. 771, L14 (2013)

L.-J. Guo, Y.-M. Huang, A. Bhattacharjee, D.E. Innes, Astrophys. J. 796, L29 (2014)

W.J. Hanneman, K.K. Reeves, Astrophys. J. 786, 95 (2014)

H. Hara, T. Watanabe, L.K. Harra et al., Astrophys. J. 741, 107 (2011)

Y.M. Huang, A. Bhattacharjee, Phys. Plasmas 17, 062104 (2010)

Y.M. Huang, A. Bhattacharjee, Phys. Rev. Lett. 109, 265002 (2012)

G. Huang, J. Lin, Astrophys. J. 639, L99 (2006)

Y.M. Huang, A. Bhattacharjee, E.G. Zweibel, Phys. Plasmas 17, 055707 (2010)

S.Y. Huang, A. Vaivads, Y.V. Khotyaintsev, M. Zhou, H.S. Fu, A. Retinó et al., Geophys. Res. Lett. 39, L11103 (2012)

S. Ichimaru, Astrophys. J. 202, 524 (1975)

D.E. Innes, D.E. McKenzie, T.J. Wang, Sol. Phys. 217, 267 (2003a)

D.E. Innes, D.E. McKenzie, T.J. Wang, Sol. Phys. 217, 247 (2003b)

D.E. Innes, L. Guo, A. Bhattacharjee, Y.M. Huang (2014)

A. Isavnin, A. Vourlidas, E.K.J. Kilpua, Sol. Phys. 284, 203 (2013)

P.A. Isenberg, T.G. Forbes, P. Demoulin, Astrophys. J. 417, 368 (1993)

M. Karlický, F. Fárník, S. Krucker, Astron. Astrophys. 419, 365 (2004)

M. Karlický, P. Zlobec, H. Mészárosová, Sol. Phys. 261, 281 (2010)

M. Karlický, M. Bárta, Astrophys. J. 733, 107 (2011)

M. Karlický, B. Kliem, Sol. Phys. 266, 71 (2010)

J.T. Karpen, S.K. Antiochos, C.R. DeVore, Astrophys. J. 760, 81 (2012)

B. Kliem, T. Török, Phys. Rev. Lett. 96, 255002 (2006)

B. Kliem, M. Karliký, A.O. Benz, Astron. Astrophys. 360, 715 (2000)

B. Kliem, M.G. Linton, T. Török, M. Karliký, Sol. Phys. 266, 39 (2010)

B. Kliem, J. Lin, T.G. Forbes, E.R. Priest, T. Török, Astrophys. J. 789, 46 (2014)

Y.-K. Ko, J.C. Raymond, J. Lin, G. Lawrence, J. Li, A. Fludra, Astrophys. J. 594, 1068 (2003)

Y.-K. Ko, J.C. Raymond, B. Vršnak, E. Vujić, Astrophys. J. 722, 625 (2010)

J.L. Kohl, R. Esser, L.D. Gardner, S. Habbal, P.S. Daigneau, E.F. Dennis et al., Sol. Phys. 162, 313 (1995)

J.L. Kohl, N. Giancarlo, S.R. Cranmer, J.C. Raymond, Astron. Astrophys. Rev. 13, 31 (2006)

S. Kolomański, M. Karlický, Astron. Astrophys. 475, 685 (2007)

R.A. Kopp, G.W. Pneuman, Sol. Phys. 50, 85 (1976)

G. Kowal, A. Lazarian, E.T. Vishniac, K. Otmianowska-Mazur, Astrophys. J. 700, 63 (2009) 
S. Krucker, M. Battagalia, P.J. Cargill, L. Fletcher, H.S. Hudson, A.L. MacKinnon et al., Astron. Astrophys. Rev. 16, 155 (2008)

R.M. Kulsrud, Earth Planets Space 53, 417 (2001)

E. Landi, J.C. Raymond, M.P. Miralles, H. Hara, Astrophys. J. 711, 75 (2010)

E. Landi, J.C. Raymond, M.P. Miralles, H. Hara, Astrophys. J. 751, 21 (2012)

A. Lazarian, E.T. Vishniac, Astrophys. J. 517, 700 (1999)

A. Lazarian, L. Vlahos, G. Kowal, H. Yan, A. Beresnyak, E.M. de Gouveia Dal Pino, Space Sci. Rev. 173, 557 (2012)

A. Lazarian, G. Eyink, E. Vishniac, G. Kowal, arXiv:1407.6356 (2014)

Y. Li, W. Gan, Astrophys. J. 629, L137 (2005)

Y. Li, W. Gan, Astrophys. J. 644, L97 (2006)

Y. Li, J. Lin, Acta Astron. Sin. 50, 271 (2009)

Y. Li, J. Lin, Chin. Astron. Astrophys. 34, 48 (2010)

Y. Li, J. Lin, Sol. Phys. 279, 91 (2012)

J. Li, D. Jewitt, B. LaBonte, Astrophys. J. 539, L67 (2000)

C.K. Li, F.H. Seguin, J.A. Frenje, J.R. Rygg, R.D. Petrasso, R.P.J. Town et al., Phys. Rev. Lett. 99, 055001 (2007)

G. Li, X. Kong, G. Zank, Y. Chen, Astrophys. J. 769, 22 (2013a)

Y. Li, H.D. Winter, N.A. Murphy, J. Lin, N. Wu, Publ. Astron. Soc. Jpn. 65, 101 (2013b)

J. Lin, Chin. J. Astron. Astrophys. 2, 539 (2002)

J. Lin, Sol. Phys. 222, 115 (2004)

J. Lin, T.G. Forbes, J. Geophys. Res. 105, 2375 (2000)

J. Lin, W. Soon, New Astron. 47, 53 (2004)

J. Lin, T.G. Forbes, E.P. Priest, T.N. Bungey, Sol. Phys. 159, 275 (1995)

J. Lin, T.G. Forbes, P.A. Isenberg, P. Démoulin, Astrophys. J. 504, 1006 (1998)

J. Lin, A.A. van Ballegooijen, T.G. Forbes, J. Geophys. Res. 107, 1438 (2002)

J. Lin, W. Soon, S.L. Baliunas, New Astron. Rev. 47(2), 53 (2003)

J. Lin, J.C. Raymond, A.A. van Ballegooijen, Astrophys. J. 602, 422 (2004)

J. Lin, Y.K. Ko, L. Sui et al., Astrophys. J. 622, 1251 (2005)

J. Lin, S. Mancuso, A. Vourlidas, Astrophys. J. 649, 1110 (2006)

J. Lin, J. Li, T.G. Forbes, Y.K. Ko, J.C. Raymond, A. Vourlidas, Astrophys. J. 658, L123 (2007)

J. Lin, S.R. Cranmer, C.J. Farrugia, J. Geophys. Res. 113(A11), A11107 (2008)

J. Lin, J. Li, Y.K. Ko, J.C. Raymond, Astrophys. J. 693, 1666 (2009)

A.G. Ling, D.F. Webb, J.T. Burkepile, E.W. Cliver, Astrophys. J. 784, 91 (2014)

J.A. Linker, Z. Mikić, R. Lionello, P. Riley, T. Amari, D.J. Odrstrcil, Plasma Phys. 10(5), 1971 (2003)

Y. Litvinenko, Astrophys. J. 462, 997 (1996)

R. Liu, Mon. Not. R. Astron. Soc. 434, 1309 (2013)

R. Liu, J. Lee, T. Wang et al., Astrophys. J. 723, L28 (2010)

Y.H. Liu, J.F. Drake, M. Swisdak, Phys. Plasmas 19, 022110 (2012)

W. Liu, Q. Chen, V. Petrosian, Astrophys. J. 773, 166 (2013)

D.W. Longcope, T.G. Forbes, Sol. Phys. 289, 2091 (2014)

N.F. Loureiro, A.A. Schekochihin, S.C. Cowley, Phys. Plasmas 14, 100703 (2007)

N.F. Loureiro, R. Samtaney, A.A. Schekochihin, D.A. Uzdensky, Phys. Plasmas 19, 042303 (2012)

B.J. Lynch, S.K. Antiochos, P.J. MacNeice, T.H. Zurbuchen, L.A. Fisk, Astrophys. J. 617, 589 (2004)

B.J. Lynch, S.K. Antiochos, C.R. DeVore, J.G. Luhmann, T.H. Zurbuchen, Astrophys. J. 683, 1192 (2008)

B.J. Lynch, Y. Li, A.F.R. Thernisien, E. Robbrecht, G.H. Fisher, J.G. Luhmann, A. Vourlidas, J. Geophys. Res. 115, A07106 (2010)

G. Mann, A. Warmuth, Astron. Astrophys. 528, A104 (2011)

G. Mann, A. Warmuth, H. Aurass, Astron. Astrophys. 494, 669 (2009)

P.C.H. Martens, Astrophys. J. 330, L131 (1988)

P.C.H. Martens, N.P.M. Kuin, Sol. Phys. 122, 263 (1989)

P.C.H. Martens, A. Young, Astrophys. J. Suppl. Ser. 73, 333 (1990)

S. Masuda, T. Kosugi, H. Hara, S. Tsuneta, Y. Ogawara, Nature 371, 495 (1994)

D.E. McKenzie, Sol. Phys. 195, 381 (2000)

D.E. McKenzie, Astrophys. J. 766, 39 (2013)

D.E. McKenzie, H.S. Hudson, Astrophys. J. 519, L93 (1999)

Z. Mei, C. Shen, N. Wu et al., Mon. Not. R. Astron. Soc. 425, 2824 (2012)

Z. Mikić, J.A. Linker, Astrophys. J. 430, 898 (1994)

Z. Mikić, D.C. Barnes, D.D. Schnack, Astrophys. J. 328, 830 (1988)

R.O. Milligan, R.T.J. McAteer, B.R. Dennis, C.A. Young, Astrophys. J. 713, 1292 (2010)

R.L. Moore, A.C. Sterling, H.S. Hudson, J.R. Lemen, Astrophys. J. 552, 833 (2001) 
N.A. Murphy, A.K. Young, C.C. Shen, J. Lin, L. Ni, Phys. Plasmas 20, 061211 (2013)

N. Narukage, K. Shibata, Astrophys. J. 637, 1122 (2006)

L. Ni, K. Germaschewski, Y.M. Huang et al., Phys. Plasmas 17, 052109 (2010)

L. Ni, I.I. Roussev, J. Lin, U. Ziegler, Astrophys. J. 758, 20 (2012a)

L. Ni, U. Ziegler, Y.M. Huang, J. Lin, Z. Mei, Phys. Plasmas 19, 072902 (2012b)

L. Ni, J. Lin, N.A. Murphy, Phys. Plasmas 20, 061206 (2013)

L. Ni, B. Kliem, J. Lin, N. Wu, Astrophys. J. 799, 79 (2015)

P.N. Nilson, L. Willingale, M.C. Kaluza, C. Kamperidis, S. Minardi, M.S. Wei et al., Phys. Rev. Lett. 97, 255001 (2006)

P.N. Nilson, L. Willingale, M.C. Kaluza, C. Kamperidis, S. Minardi, M.S. Wei et al., Phys. Plasmas 15, 092701 (2008)

N. Nishizuka, K. Shibata, Phys. Rev. Lett. 110, 051101 (2013)

G. Noci, J.L. Kohl, G.L. Withbroe, Astrophys. J. 315, 706 (1987)

M. Opher, J.F. Drake, M. Swisdak et al., Astrophys. J. 734, 71 (2011)

S. Patsourakos, A. Vourlidas, Astron. Astrophys. 525, 27 (2011)

S. Patsourakos, A. Vourlidas, G. Stenborg, Astrophys. J. 764, 125 (2013)

H.E. Petschek, in The Physics of Solar Flares, ed. by W.N. Hess (1964), p. 425. NASA Spec. Publ. SP-50

G. Poletto, S.T. Suess, A. Bemporad, N.A. Schwadron, H.A. Elliott, T.H. Zurbuchen, Y.K. Ko, Astrophys. J. 613, L173 (2004)

E.R. Priest, Solar Magnetohydrodynamics. Geophys. Astrophys. Monogr., vol. 21 (Reidel, Dordrecht, 1982)

E.R. Priest, Rep. Prog. Phys. 48, 955 (1985)

E.R. Priest, Astrophys. Space Sci. 264, 77 (1988)

E.R. Priest, T.G. Forbes, Magnetic Reconnection: MHD Theory and Applications (Cambridge University Press, Cambridge, 2000)

E.R. Priest, T.G. Forbes, Astron. Astrophys. Rev. 10, 313 (2002)

P.L. Pritchett, Y.C. Lee, J.F. Drake, Phys. Fluids 23, 1368 (1980)

J.C. Raymond, A. Ciaravella, D. Dobrzycka et al., Astrophys. J. 597, 1106 (2003)

K.K. Reeves, L. Golub, Astrophys. J. 727, L52 (2011)

K.K. Reeves, T.B. Guild, W.J. Hughes et al., J. Geophys. Res. 113(A7), A00B02 (2008a)

K.K. Reeves, D.B. Seaton, T.G. Forbes, Astrophys. J. 675, 868 (2008b)

K.K. Reeves, J.A. Linker, Z. Mikić, T.G. Forbes, Astrophys. J. 721, 1547 (2010)

K.K. Reeves, S.E. Gibson, T.A. Kucera, H.S. Hudson, R. Kano, Astrophys. J. 746, 146 (2012)

B.A. Remington, R.P. Drake, D.D. Ryutov, Rev. Mod. Phys. 78, 755 (2006)

A. Retinó, D. Sundkvist, A. Vaivads, F.S. Mozer, M. André, C.J. Owen, Nat. Phys. 3, 236 (2007)

A. Retinó, R. Nakamura, A. Vaivads, Y. Khotyaintsev, T. Hayakawa, K. Tanaka et al., J. Geophys. Res. 113, 12215 (2008)

P. Riley, R. Lionello, Z. Mikić et al., Astrophys. J. 655, 591 (2007)

I.W. Roxburgh, Mon. Not. R. Astron. Soc. 132, 201 (1966)

D.D. Ryutov, R.P. Drake, B.A. Remington, Astrophys. J. Suppl. Ser. 127, 456 (2000)

P. Saint-Hilaire et al., Astrophys. J. 696, 941 (2009)

S.L. Savage, D.E. McKenzie, Astrophys. J. 730, 98 (2011)

S.L. Savage, D.E. McKenzie, K.K. Reeves et al., Astrophys. J. 722, 329 (2010)

S.L. Savage, D.E. McKenzie, K.K. Reeves, Astrophys. J. 747, L40 (2012a)

S.L. Savage, G. Holman, K.K. Reeves, D.B. Seaton, D.E. McKenzie, Y. Su, Astrophys. J. 754, 13 (2012b)

B. Schmieder, T.G. Forbes, J.M. Malherbe, M.E. Machado, Astrophys. J. 317, 956 (1987)

B. Schmieder, P. Demoulin, G. Aulanier, Adv. Space Res. 51, 1967 (2013)

R.B. Scott, D.W. Longcope, D.E. McKenzie, Astrophys. J. 776, 54 (2013)

D.B. Seaton, T.G. Forbes, Astrophys. J. 701, 348 (2009)

V.D. Shafranov, Rev. Plasma Phys. 2, 103 (1966)

M.A. Shay, J.F. Drake, J.P. Eastwood, T.D. Phan, Phys. Rev. Lett. 107(6), 065001 (2011)

N.R. Sheeley, Y.M. Wang, Astrophys. J. 579, 874 (2002)

N.R. Sheeley, Y.M. Wang, Astrophys. J. 655, 1142 (2007)

N.R. Sheeley, H.P. Warren, Y.M. Wang, R.A. Howard, J. Geophys. Res. 104, 24739 (1999)

N.R. Sheeley, H.P. Warren, Y.M. Wang, Astrophys. J. 616, 1224 (2004)

C. Shen, J. Lin, N.A. Murphy, Astrophys. J. 737, 14 (2011)

C. Shen, J. Lin, N.A. Murphy, J.C. Raymond, Phys. Plasmas 20, 072114 (2013)

K. Shibata, T. Magara, Living Rev. Sol. Phys. 8, 6 (2011)

K. Shibata, S. Tanuma, Earth Planets Space 53, 473 (2001)

D. Shiota, H. Isobe, P.F. Chen, T. Yamamoto, T. Sakajiri, K. Shibata, Astrophys. J. 634, 663 (2005)

G.M. Simnett, Astron. Astrophys. 416, 759 (2004)

E.C. Sittler Jr., M. Guhathakurta, Astrophys. J. 523, 812 (1999) 
M. Skender, G. Lapenta, Phys. Plasmas 17, 2905 (2010)

H. Song, X. Kong, Y. Chen et al., Sol. Phys. 276, 261 (2012)

J.A. Stamper, B.H. Ripin, Phys. Rev. Lett. 34, 138 (1975)

H.R. Strauss, Phys. Fluids 29, 3668 (1986)

H.R. Strauss, Astrophys. J. 326, 412 (1988)

Y. Su, A.M. Veronig, G.D. Holman, B.R. Dennis, T. Wang, M.G. Temmer, W. Gan, Nat. Phys. 9, 489 (2013)

L. Sui, G.D. Holman, Astrophys. J. 596, L251 (2003)

L. Sui, G.D. Holman, B.R. Dennis, Astrophys. J. 612, 546 (2004)

L. Sui et al., Astrophys. J. 633, 1175 (2005)

L. Sui, G.D. Holman, B.R. Dennis, Astrophys. J. 645, L157 (2006)

R. Susino, A. Bemporad, S. Krucker, Astrophys. J. 777, 93 (2013)

Z. Švestka, Solar Flares (Springer, Berlin, 1976), p. 168

Z. Švestka, Sol. Phys. 169, 403 (1996)

Z. Švestka, E.W. Cliver, Eruptive Solar Flares (Springer, New York, 1992)

Z. Švestka, J.M. Fontenla, M.E. Machado et al., Sol. Phys. 108, 237 (1987)

Z. Švestka, F. Fárník, H.S. Hudson, P. Hick, Sol. Phys. 182, 179 (1998)

S. Takasao, A. Asai, H. Isobe, K. Shibata, Astrophys. J. 745, L6 (2012)

V.S. Titov, P. Démoulin, Astron. Astrophys. 351, 707 (1999)

T. Török, B.H. Kliem, Astrophys. J. 630, L97 (2005)

D.A. Uzdensky, N.F. Loureiro, A.A. Schekochihin, Phys. Rev. Lett. 105, 235002 (2010)

V.M. Vasyliunas, Rev. Geophys. Space Phys. 13, 303 (1975)

A.M. Veronig, M. Karliký, B. Vršnak et al., Astron. Astrophys. 446, 675 (2006)

E.T. Vishniac, S. Pillsworth, G.L. Eyink, G. Kowal, A. Lazarian, S. Murray, Nonlinear Process. Geophys. 19, 605 (2012)

B. Vršnak, A. Warmuth, M. Temmer, A. Veronig, J. Magdelenić, A. Hillaris, M. Karlický, Astron. Astrophys. 448, 739 (2006)

B. Vršnak, G. Poletto, E. Vujić et al., Astrophys. J. 499, 905 (2009)

H.P. Warren, C.M. O’Brien, N.R. Sheeley, Astrophys. J. 742, 92 (2011)

D.F. Webb, E.W. Cliver, J. Geophys. Res. 100, 5853 (1995)

D.F. Webb, J. Burkepile, T.G. Forbes, P. Riley, J. Geophys. Res. 108(A12), SSH6-1 (2003)

L.M. Widrow, Rev. Mod. Phys. 74, 775 (2002)

P. Wood, T. Neukirch, Sol. Phys. 226, 73 (2005)

P.F. Wyper, D.I. Pontin, Phys. Plasmas 21, 082114 (2014a)

P.F. Wyper, D.I. Pontin, Phys. Plasmas 21, 102102 (2014b)

X. Yan, Z. Xue, J. Liu, L. Ma, D. Kong, Z. Qu, Z. Li, Astrophys. J. 782, 67 (2014)

J. Yang, Y. Jiang, Y. Bi, H. Li, J. Hong, D. Yang, R. Zheng, B. Yang, Astrophys. J. 749, 12 (2012)

M.A. Yates et al., Phys. Rev. Lett. 49, 1702 (1982)

T. Yokoyama, K. Shibata, Astrophys. J. 436, L197 (1994)

T. Yokoyama, K. Shibata, Astrophys. J. 474, L61 (1997)

T. Yokoyama, K. Shibata, Astrophys. J. 494, L113 (1998)

T. Yokoyama, K. Shibata, Astrophys. J. 549, 1160 (2001)

T. Yokoyama, K. Akita, T. Morimoto et al., Astrophys. J. 546, L69 (2001)

Y. Zhang, J. Wang, Astrophys. J. 663, 592 (2007)

Y. Zhang, Y. Hu, J. Wang, Astrophys. J. 626, 1096 (2005)

Y. Zhang, J. Wang, Y. Hu, Astrophys. J. 641, 572 (2006)

V.V. Zharkova et al., Space Sci. Rev. 159, 357 (2011)

J.Y. Zhong, Y.T. Li, X.G. Wang, J.Q. Wang, Q.L. Dong, C.J. Xiao et al., Nat. Phys. 6, 984 (2010)

E.G. Zweibel, M. Yamada, Annu. Rev. Astron. Astrophys. 47, 291 (2009) 